Tittle for the lettering along the back of the bound copies.

DIVIDING FLOW IN AN OPEN CHANNEL - LAW 


\section{DIVIDING FLOW IN AN OPEN}

CHANNEL

By

Shiu Wai Law

A thesis submitted to the Faculty of Graduate Studies and Research in partial fulfilment of the requirements for the degree of Master of Engineering.

Department of Civil Engineering and Applied Mechanics, Mc Gill University, Montreal. 


\section{SUMMARY}

An overall view is given of a dividing flow with mean subcritical flows in all channels. Experimental observations of reverse flows or recirculation regions, hydraul ic jumps and local flows at the junction are described in detail. Photographs showing these phenomena under different flow ratios are also presented. A surface discontinuity at the junction which is not known previously is also mentioned.

The channels used in the experiment are of rectangular section and have horizontal beds, the branch channel being set at right angles midway along the straight main channel. Discharge and depth measurements are taken with an orifice meter and a point gauge. Photographs showing hydraulic jumps, reverse flows and flow conditions have been taken with a $35 \mathrm{~mm}$. camera at different angles. Sketches showing the reverse flow regions in the main channel extension and the branch are presented.

Based on simplified principles of momentum and energy, a theoretical analysis of the flow in this model is given. The consideration of the flow in the main channel extension is possible by introducing plausible assumptions into both principles. For the flow in the branch it is not possible to make an a priori estimate of the energy loss, and the momentum equation is useful only when a contraction coefficient is introduced. The contraction coefficient accounts for the presence of the extensive reverse 
flow regions in the branch channel. No such contraction coefficient is required to describe the flow in the main channel extension since the region of the reverse flow is relatively small.

Finally the momentum theory is used to correlate the experimental results of Grace ${ }^{(3)}$ for various angles of intersection at the junction. It is found that the contraction coefficient is a function of the Froude numbers of the flows in the main channel and in the branch, the distribution of the inflow into the two streams downstream of the junction, and the angle of intersection. The contraction coefficient is essential in understanding the problem of a dividing flow in an open channel. Without a thorough knowledge of its development under different flow conditions, a theoretical analysis is difficult. To get the insight of each practical problem of a dividing flow in an open channel, a model test is strongly recommended. 


\section{ACKNOWLED GEMENTS}

The author is deeply indebted to $\mathrm{Dr}$. A.J. Reynolds for his guidance and advice throughout the course of this work.

Thanks are due to Mr. Narayanan Rangaswami for his ass istance in connection with the study of the flow in the junction.

The financial support of the National Research Council of Canada is gratefully acknowledged. 


\section{TABLE OF CONTENTS}

SUMMARY

ACKNOWLEDGEMENTS

TABLE OF CONTENTS

NOTATION

1. INTRODUCTION

1.1 Dividing Flow in an Open Channel

1.2 Literature Survey

1.3 Scope

2. Overall View of a Dividing Flow

2.1 Intorduction

2.2 Main Channel

2.3 Branch Channel

2.4 Main Channel Extension

8

2.5 Reverse Flows

9

2.6 Hydraulic Jumps

3. Apparatus and Experimental Procedure

\subsection{Apparatus \\ 3.2 Experimental Procedure}

4. Theoretical Analys is

4.1 Intorduction

4.2 Main Channel Extension

4.2. 1 Momentum Principle

4.2.2 Energy Principle

4.3 Branch Channel

4.3.1 Momentum Principle 
5. Experimental Results and Discussion

5.1 Limiting Cases $\quad 27$

5.2 Overall Performance of the Main Channel Extension 28

5.2.1 Energy Loss 30

5.3 Overall Performance of the Branch Channel 32

5.4 Contraction Coefficients 33

5.5 General Review 36

6. Detailed Studies

6.1 Reverse Flow 37

6.2 Hydraulic Jump 38

6.3 Flow in the Junction 40

6.4 Surface Discontinuity 41

7. Extension of the Theory 42

8. Conclusion $\quad 51$

REFERENCES

TABLES

$\begin{array}{ll}\text { FIGURES } & 61\end{array}$

PLATES 


\section{NOTATION}

$\mathrm{C}_{\mathrm{d}}$

$d_{s}$

$d_{s} / d$

$F_{s}$

g

$\Delta \mathrm{H}$

$h_{e}$

9

$Q_{s}$<smiles>O=[SH]O</smiles>
$u_{s}$

$u_{b}^{\prime}$ the width of all channel sections

the contraction coefficient, the ratio of the active

branch width to the width of the channel section

the discharge coefficient, as def ined in Eq. 4.25

the mean depth in a channel section, taken at mid-

point of the section

the depth ratio in a channel

the Froude number of the flow in a channel, defined

by: $\quad F_{s}=u_{s} / \sqrt{g d_{s}}$

the acceleration of gravity

the energy loss across a hydraulic jump, as defined in

$\mathrm{Eq} \cdot 5.9$

the head above a weir, in Eq. 4.25

the discharge per unit of channel width, in Eq. 4.25

the discharge in a channel

the discharge ratio in a channel

the mean velocity of the flow in a channel section, neglecting the contraction effect owing to the presence of a reverse flow, defined by $Q_{s} / b d_{s}$ the effective velocity of the flow in the branch channel, taken into effect the presence of a reverse flow, defined by $Q_{b} / d_{b} c b$ or $u_{b}=c u_{b}^{\prime}$. This term is defined only in the branch channel. 
$w_{s}$ the weir height at the exit end of the channels

$\theta$ the angle of intersection between the main channel extension and the branch

$\rho \quad$ density of water

Subscripts

In any of the quantities, $d_{s}, F_{s}, Q_{s}, u_{s}$ and $w_{s}$, when no subscript is used, the term refers to the main channel, unless specified.

when $s=m$, the term refers to the main channel extension when $s=b$, the term refers to the branch when $s=i, \quad$ the term refers to the junction

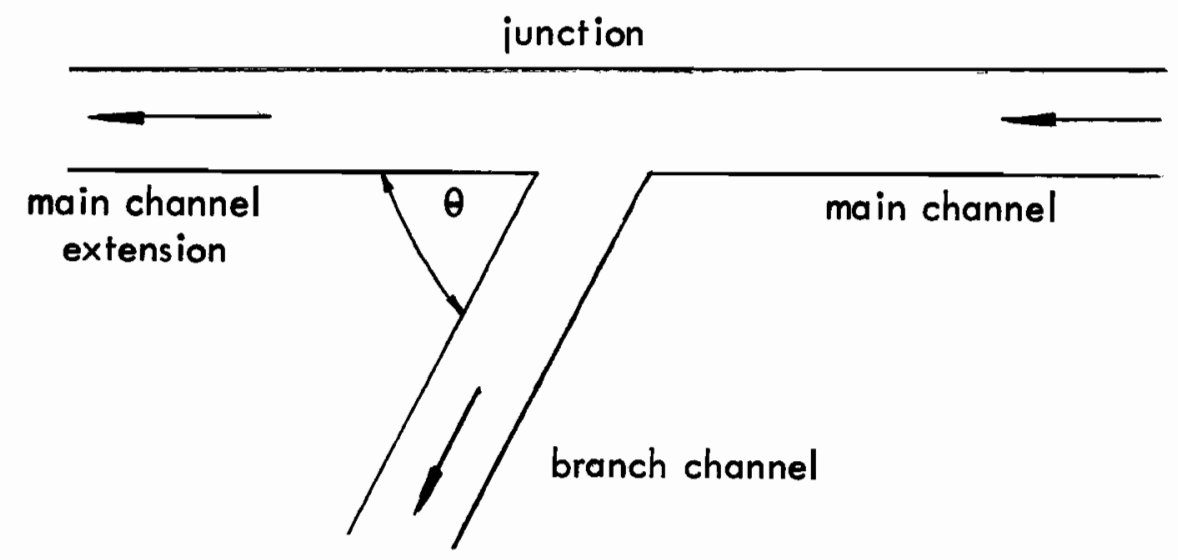




\section{INTRODUCTION}

\subsection{Dividing Flow in an Open Channel}

A dividing flow in an open channel finds many applications in fields such as river engineering, drainage, sewage treatment, and irrigation. In view of the importance of its applications in engineering fields, an attempt will be made here to present an overall view of a dividing flow. To the practical engineer, an understanding of the overall behaviour of a dividing flow may be more interesting than the realization of the local complexities of the flow. However, an insight into the details of the flow is essential in order to understand the nature of the problem.

The difficulty of a theoretical and/or even a comprehensive experimental study becomes apparent from the following consideration. It must be recognized that the walls of the channels may not be the effective boundaries of the flows. Separation along the walls may arise owing to pressure gradient and boundary effect. Hence, given a subcritical inflow into the main channel, the flows at and downstream of the junction can acquire supercritical flows even in horizontal channels. In general, the conditions of flow downstream of the junction depend primarily on the state and distribution of the inflow at the junction. The state of flow in a channel is influenced by factors such as the width of the channels, the angle of intersection of the branch, the slopes and the roughness of the several channels. Downstream of the junction, recirculation regions, due to flow separation, may form in either the main channel extension or in the branch channel or in both channels (Figure 1). In the branch channel, these recirculation regions have been observed to extend as far as the exit end of the channel. In the main channel extension, the recirculation region usually ends downstream of the junction before it reaches the exit end of the channel.

In the junction itself, the flows may be tranquil (subcritical) or rapid (supercritical) depending upon the state of the inflow and its distribution 
to the two channels. When an effective critical section is established in the junction, the flow on expanding to a wider section at the junction, will be shallow and rapid. The flow in the junction depends upon the backwater effect of the two downstream channels below the dividing junction as well as upon the dynamic conditions existing at the junction.

\subsection{Literature Survey}

In this section some of the currently available Engl ish literature in connection with the dividing flow study in an open channel are discussed. It is understood that tranquil flow in a channel means that the mean flow in the channel is tranquil. Locally, the flow may be rapid. In all cases considered thereafter, the inflow is tranquil and no flow in the junction is being considered.

A first comprehensive study of a dividing flow in an open channel was given by Taylor ${ }^{(9)}$. He suggested that for any given angle of intersection of the branch channel, it was possible to correlate the discharge ratios, the depth ratios in the branch channel with the ratio of kinetic energy head to depth in the main channel as parameters. The depth or discharge in the branch was then obtained by interpolating the experimental results.

The experimental results presented by Taylor on a $90^{\circ}$ model agreed well with the author 's results with the exception that the limits corresponding to free-overfall conditions in the two channels downstream of the junction were not defined by Taylor. The graphical representation suggested by Taylor in terms of ratios of depth and discharge did provide a very useful method in describing the dividing flow in an open channel.

In terms of the ratios of depth and discharge, Grace ${ }^{(3)}$ presented experimental results covering the cases of a dividing flow in a model with rectangular cross-section in which different ratios of the width of the straight main channel to the branch channel could be obtained and the angle of the branch to the straight channel could also be varied. 
Grace distinguished two flow regions, with and without the appearance of surface waves (hydraulic jumps). Though it was not specified explicitly, the former case corresponded to relatively slow flows in the several channels and the latter to free-overfall conditions in the channels downstream of the junction. These were the two extreme cases which a dividing flow could attain. Other intermediate flow conditions have not been examined by Grace.

In his work, it was shown experimentally that given a constant width of the several channels, the limit curve corresponded to free-overfall conditions downstream of the junction followed a general pattern for different angles of intersection. This fact indicated the feasibility to study a dividing flow for a particular angle of intersection and to generalize the results to include other angles.

After Taylor and Grace, a number of research workers studied the case of a dividing flow either directly or indirectly. Yoshimi and Stelson investigated the sewage flow from a single inlet conduit to multiple grit channels and gave a comprehensive description of the flow pattern in the junction region. A dividing flow with a tranquil (subcritical) flow in a horizontal main channel and a shooting (supercritical) flow in a sloping branch channel has also been studied by Krishnappa, Rajaratnam, and Thiruvengadam $(5,6,7,10)$. In these studies, the branch flow was treated as a flow through a side weir of zero height in De Marchi's side-weir formula and an experimental coefficient was then sought.

A problem allied to flow division is flow combination. Combining flow has been studied by a number of people, for example, Taylor and Bower $(9,2)$. In his work, Taylor assumed that 1) a uniform velocity distribution exists across the channels 2) wall friction is negligible in comparison with the pressure, gravity, and inertia forces and 3) the depths of the intersecting channels are equal immediately above the junction. Momentum balances were then set up relating the inflow and the outflow. It was found that the agreement 
between theory and experiment was quite good for cases when the intersecting angle of the channels was $135^{\circ}$ and was rather poor for cases with an intersecting angle of $45^{\circ}$. In the former case, no reversal in the inflow direction occurs. Consequently, in the former case, the flow condition in the junction is less complicated, rendering a better estimation of the force developed at the junction and resulting in a better prediction of a combining flow.
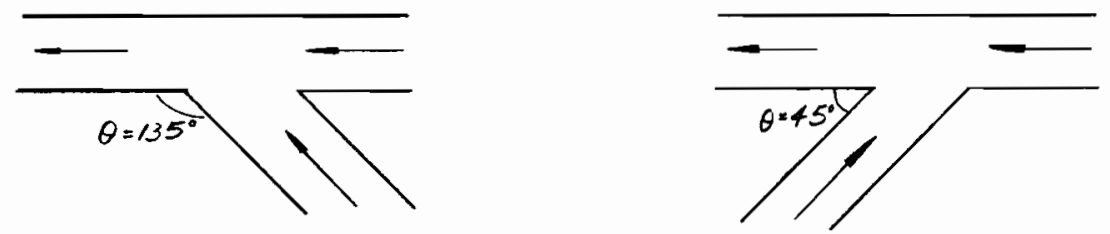

In the case of a dividing flow, the flow in the junction is expected to be less complicated when the angle of intersection is small rather than large since the inflow does not need to turn a large angle in the junction. However, this is not true due to different flow conditions in the branch, notably the phenomenon of flow separation. It is obvious that only by understanding the local phenomenon of the flow in the several channels that a dividing flow can be studied properly.

\subsection{Scope}

In this work, it is proposed to study the overall performance of a dividing flow utilizing a model in which a branch is set at right angle to the straight channel. Details of local flow conditions in the branch and in the junction are given. The flows are carefully observed to note the extent of the recirculation regions due to flow separation, and hydraulic jumps. It is hoped that enough insight into these local conditions is given that the result can be generalized for other angles of intersections. 
Chapter 1. An introduction is given to describe briefly the complexities and its applications in various fields of a dividing flow in an open channel. Literature survey and the presentation of this thesis are stated.

Chapter 2. In this chapter, the observable behaviour of the flow is presented. An overall view of the dividing flow in different channels will give an insight into the nature of the problem. The general behaviour of the reverse flow and the hydraulic jump will also be described.

Chapter 3. A description of the apparatus and a brief account of the experimental procedure will be given in this chapter.

Chapter 4. A theoretical analys is will be given separately for the flows in the main channel extension and in the branch channel. A general discussion of the theoretical results is given. Similarity of the several channels to a straight channel will also be discussed.

Chapter 5. In this chapter, the limits of the Froude numbers in the several channels will be discussed. The choice of the contraction coefficient for the flow in the branch under various flow conditions will be described in detail. The experimental and theoretical results will be compared and discussed.

Chapter 6. More detail in the analysis and description of a reverse flow, the hydraulic jumps and the flow in the junction will be given. In this chapter, the appearance of a surface discontinuity at the junction will also be described briefly.

Chapter 7. An attempt is made to extend the theory developed in Chapter 4 to include a general angle of intersection. The effect on varying the width of the branch channel is also discussed. In this chapter, the experimental results by Grace ${ }^{(3)}$ are correlated with the theory developed in this study. The variation of the contraction coefficient corresponding to different ranges of discharge distribution will be described in detail. 
Chapter 8. In this final chapter of the thesis, a conclusion will be given summing up this study of a dividing flow in an open channel. 


\section{OVERALL VIEW OF A DIVIDING FLOW}

\subsection{Introduction}

In th is chapter, the observable behaviours of a dividing flow are described. Separate sections are devoted to the description of the flow in each channel and to the introduction of the "reverse flows" or "recirculation regions", and hydraulic jumps which occur there. It should be noted that while the hydraulic jumps form a prominent surface phenomenon, the recirculation regions, although not visible, extend from the bottom of the channel to the surface of the flows. These phenomena of "reverse flows" and "hydraulic jumps" are described briefly in this chapter. More details of the ir developments under different states of flow will be given in Chapter 6 .

\subsection{Main Channel}

This is the part of the straight channel between the stilling basin and the junction. The flow in this channel is always tranquil and the surface is generally smooth. Near the junction, the flow surface slopes down gradually towards the junction where the flow is faster and the depth is shallower. In this channel, no hydraulic jumps or reverse flows are observed.

\subsection{Branch Channel}

There are many possible flow conditions in the branch channel. In all cases, it can be observed that there is an eddy or a region of recirculation existing at the upstream corner of the junction due to flow separation (Fig. 2.3). The extent of this recirculation region in the downstream direction and in the

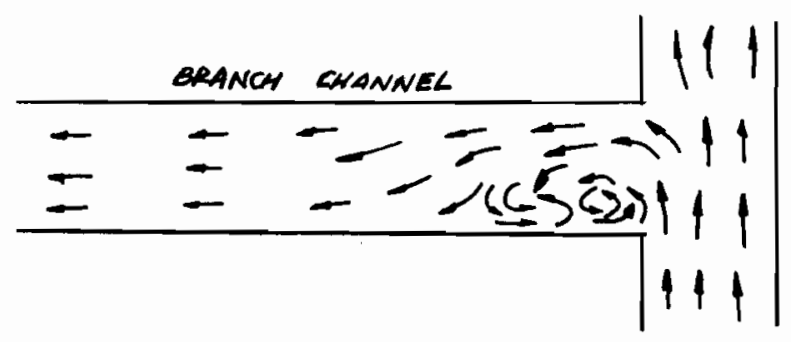

Fig. 2.3 Flow separation at corner of junction. 
cross-channel direction depends on the state of the inflow, the percentage of the total discharge in the branch and the depth of the flow in the branch. For large discharge ratios which correspond to relatively greater depth in this channel, there is no reverse flow observable (while a reverse flow may be observed in the main channel extension) and the surface in this channel is relatively smooth. However, for small discharge ratios, the presence of a reverse flow extending both in length and width into the downstream direction can be observed. (Plate 3).

The formation of hydraulic jumps in the branch channel is a function of the discharge ratios and the depth of the branch. Hydraulic jumps usually take the form of criss-cross waves occupying about half the width of the branch and extend to about half the length near the right hand wall of the channel (looking downstream). At the same time, there may be a reverse flow along the left hand wall of the channel (Figure 4.3.1).

Reverse flows play a significant role in determining the state of flow in the branch. Flows moving upstream occupying about half the width of the branch have been observed for small discharge ratios. It is difficult to determine the active conveying width of the branch which affects the determination of the state of flow accordingly. More detail of the presence of a reverse flow will be given in appropriate sections.

\subsection{Main Channel Extension}

In the case when the discharge from this channel is small, the surface of the flow is relatively smooth and the flow can be observed to separate at the junction from the wall opposite to the junction. A recirculation region is thus generated in the main channel extension. Generally, this recirculating region ends downstream at a distance of about three times its width. Across the channel, its extent will be limited to less than one-third the width of the channel (Plate 2).

On the other hand, when more than fifty percent of the total discharge is passing through this channel, standing wave formation can be observed. The standing waves are manifestations of hydraulic jumps which are formed after the flow becomes supercritical at the junction. In this way, the shooting flow at the junction is transformed to subcritical condition on entering the main channel extension. 


\subsection{Reverse Flows}

It is important to note that the term "reverse flow" has been used in this thesis to designate the flow region along the wall or walls where the flow direction is opposite to the net flow in the channel. In reality, this region of reverse flow composes part of the recirculation region or eddying region caused by a flow separation. In this work, the phrase "reverse flow" is used to denote that part of the recirculation region in which the flow direction is opposite to the general flow direction. The presence of a narrow region of reverse flow extending as far as the exit end of the branch channel has been observed in this work. This is equivalent to saying that a recirculation region extends from the corner where the flow turns, along the wall to the exit end of the channel. Alternatively, the term "recirculation region" is used when the eddying flows form a distinct part of the flow.

Fig. 2.5 Reverse flow in branch channel

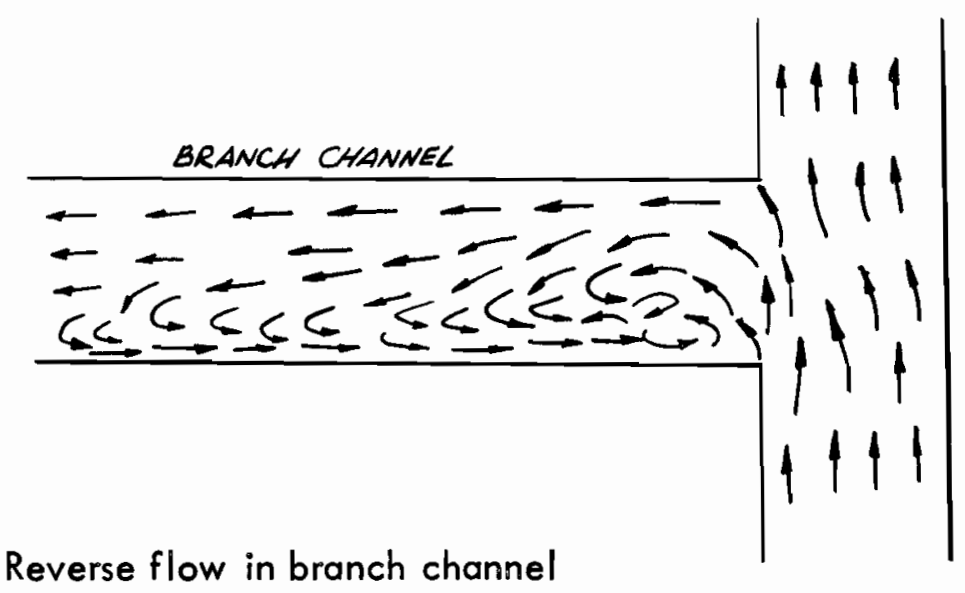

At high flow rate in the branch, no reverse flow is observed there except the eddying region at the corner as the flow separates. When the discharge ratio in the branch falls to 0.2 , this eddying region has grown downstream about four times the width of the channel (Figure 2.5). Thereafter, this recirculation region grows in length ropidly for a relatively small change in the discharge ratio. This growth continues until the exit end of the channel is reached. On further decreasing the discharge in the branch, the recirculation region on the left hand side (looking downstream) gradually shortens while another recirculation region is formed on the right hand side. As the flow decreases further, the left reverse flow region grows shorter and wider and the right reverse flow region widens and grows 
upstream. When there is no flow passing through the branch, recirculation regions fill the entire channel (Figure 16a to g).

When the flows in all channels are relatively slow moving, there is no reverse flow occurring in any channel except the small eddying region at the upstream corner of the branch due to flow separation.

In general, the "reverse flow" region in the main channel extension is not as extensive as that in the branch. Usually the flow reattaches to the wall and seldom reaches the exit end of the channel. In Figure $17 \mathrm{a}$ to $\mathrm{h}$, the development of the recirculation region in the main channel extension is sketched.

\subsection{Hydraulic Jumps}

It has been observed that hydraulic jumps can be found in a channel only when the discharge ratio is high and the depth of flow is relatively shallow. It has been found that hydraulic jumps in the rapid section of the branch channel appear as criss-cross waves and no simple pattern can be observed. On the other hand, the hydraulic jumps appearing in the main channel extension have a simple and two-dimensional pattern in most cases (Plates 4 and 5).

On striking the downstream corner of the junction, the fast inflow will be reflected and deflected into the ma in channel extension. Surface ripples are formed similar to those formed on the bow of a boat sail ing in water. In this thesis, these surface ripples are called "bow waves" (Figure 2.6a). As the discharge ratio

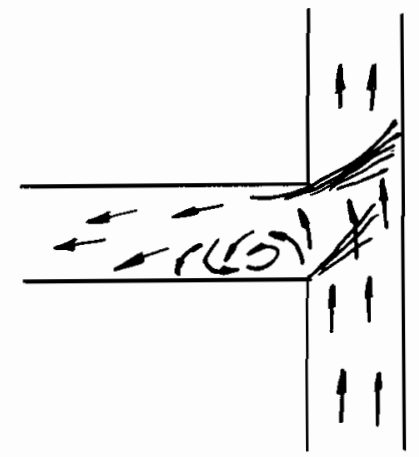

Fig. 2.6a Bow Waves

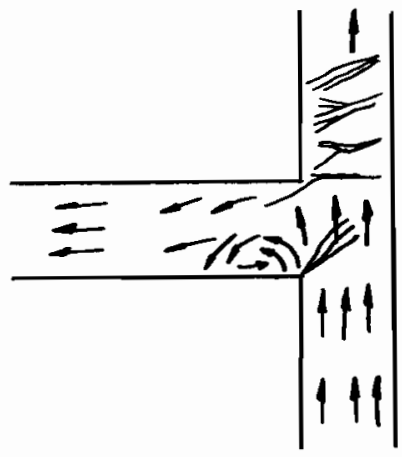

Fig. 2.6b Undular Jumps

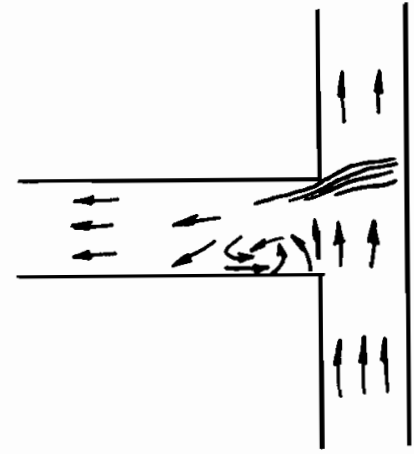

Fig. 26c Weak Jump 
of the main channel extension increases the flow at the junction accelerates and undular jumps start to form. Standing waves pattern can be distinguished in the main channel extension (Figure 2.6b). As the flow into this channel increases further, a simple hydraulic jump called weak jump is formed and the amplitudes of the standing waves diminish (Figure 2.6c). With further increase of flow into this channel, undular jumps are formed aga in and the standing waves pattern reappears. When more than ninety percent of the total inflow passes into the main channel extension, no hydraulic jump will be formed here. Under this condition, the slow flow in the branch channel behaves as if it were an elastic boundary along a portion of the wall of a straight channel. This flexible boundary will cause only a slight disturbance in the straight channel; no hydraulic jump is anticipated since the flow is in a subcritical state. 


\section{APPARATUS AND EXPERIMENTAL PROCEDURE}

\subsection{Apparatus}

In this study of the dividing flow problem in an open channel, horizontal channels with rectangular sections were used. The bottom of the channels was formed of standard structural channels, 8 inches wide in all cases. The straight main channel was 12 feet in length and the branch was 6 feet in length. The branch was set at right angles to the main channel 6 feet from the inlet. The walls were constructed of $\frac{1}{2}$ inch clear Acrylic plastic (plexiglass) plates in order to facilitate visual observation. The corners of the walls at the junction were abrupt right angles. (Figure 1 and Plate 1 ).

The inlet flow was controlled by means of a standard 3-inch gate valve. A pipe orifice meter was used to measure the inlet flow rate. The water was then passed into a stilling basin 4 feet $\times 2 \frac{1}{2}$ feet $\times 3 \frac{1}{2}$ feet. A funnel-shaped outlet with wire screen across it allowed smooth flow to be delivered into the main channel. The flow was then divided at the junction. A vertical sliding gate was installed on the exit end of each channel in order to regulate the discharge and the depth of water. All depth measurements were taken with a point gauge. The flow of the branch channel was measured as the volume discharge into a rectangular tank in a measured time interval. The discharge in the main channel extension was simply obtained by subtracting the volume discharge of the branch channel from the total discharge measured from the calibrated rating curve of the pipe orifice meter.

The direction of the surface flow was indicated with chalk powder and paper debris from IBM key punch. The bottom flow was indicated with potassium permanganate. Occasionally, chains of plastic beads were used as direction indicators.

For the study of the local flow conditions in the junction, a camera was mounted vertically above the junction. Chalk powder was sprayed on the surface upstream of the junction. Pictures of the flow were taken. In this way, the direction of flows and the relative surface velocities could be determined from the relative length of the streaks of the chalk powder left on the pictures. 


\subsection{Experimental Procedure}

The experiments were done in several stages. Initially a series of preliminary tests were run. From these tests, the general behaviour of the flow was determined. Conditions under which reverse flows or hydraulic jumps could be observed were noted and the ranges of total discharge and discharge distributions in all channels were determined. The calibration of the orifice meter was also checked frequently to ensure proper performance.

The principal part of the experiment was executed in a systematic manner to allow permutations of discharge ratios and depth. The total discharge was controlled by regulating the gate valve and the gate positions were varied to control the depths. The mean depth of each channel was taken at a station 34 inches from the centre of the junction. Depth measurements at these stations have been checked in every test to yield either a minimum or an asymptotic value. The former check ensured that the stations in both the main channel extension and the branch channel were not too close to a hydraulic jump if it occurred and the latter check ensured that these stations were free from the back water effect from the ends of the channels.

A total of more than 150 cases have been examined. The total discharge ranged from $0.08 \mathrm{cfs}$ to the maximum capacity of the model of $0.22 \mathrm{cfs}$. It has been found convenient to present the results in two parts: one pertaining to the flow in the branch and the other pertaining to that in the main channel extension.

For all the channels, the results are presented in terms of mean Froude numbers. For this case of a fixed geometry, the only parameter necessary is the discharge ratio.

In a dividing flow model, constant discharge ratios are very difficult to obtain. In this work, different discharge ratios are obtained by varying the gate positions, or the total inflow, or a combination of these variations. In presenting the experimental results, curves of constant discharge ratios are then extrapolated from these experimental points.

It is found convenient to present an alternate graphical representation of the flow. The discharge ratios, $\frac{Q_{b}}{Q}$, are plotted against the depth ratios, $\frac{d_{b}}{d}$, using the mean Froude number of the flow in the main channel, $F$, as a parameter. In 
this plot, the observable behaviours of the flow can be presented at a glance and the geometrical limits of the models can also be defined.

In this thesis, the term tranquil (or subcritical) flow refers to cases when the mean Froude number in a channel is less than unity. The mean Froude number is based on an average velocity and a mean depth of the flow across the channel. Locally, the flow may be rapid (or supercritical), or even a reverse flow condition may exist.

The flow conditions in each channel were carefully observed. For each test, a sketch was made to note the extent of the reverse flow region and whether or not hydraulic jumps were formed. These sketches were studied. The observation and study made it possible to understand the development of reverse flow regions and the hydraulic jumps relating to different discharge distributions.

The study of the local flow conditions in the junction was then undertaken to examine the changing states of the flow from subcritical to supercritical. The development of the critical section across the junction has great importance in determining the nature of the branch flow particularly when the inflow from the main channel is fast.

Three sample cases were chosen, ranging from low Froude number to high Froude number in the main channel. Pictures of the flow in the junction were taken. In the negatives of the pictures, the chalk powder sprayed on the surface showed up as black streaks. From these negatives the flow direction and the relative surface flow velocity were then determined. Depth measurements were taken at one-inch grid spacing in the junction. The Froude numbers, defined as $F=\frac{U}{\sqrt{g d}}$, covering the whole junction were computed. Contours of constant Froude numbers were then drawn to show the changes in the junction. It was found that a critical section across the whole junction could be formed only when the inflow Froude number was relatively high.

The study of the formation of standing waves was carried out in a similar fashipn except that more detail of the surface profile along the main channel extension was noted. Depth measurements were taken along the channel. Wave crest and trough were carefully noted. The wave length in each case was also observed. 


\section{THEORETICAL ANALYSIS}

\subsection{Introduction}

If the effect of viscosity is neglected, the flow in an open channel is governed by hydrostatic pressure and inertia forces. The ratio of the inertia forces to gravity forces can be expressed as a dimensionless quantity known as a Froude number. Froude number, $F$, is def ined as $F=\frac{U}{\sqrt{g d}}$, which can be written as $F=\frac{Q}{b g^{\frac{1}{2}} d^{\frac{3}{2}}}$, where $u$ is the velocity, $d$ the depth, $Q$ the discharge, $b$ the width of the channel and $g$ the acceleration of gravity.

In this model study of fixed geometry, the discharge in a channel, $Q_{s}$, can be expected to be a function of the total discharge, $Q$, the flow depth in the ma in channel, $d$, the depth of flow in the channel in question, $d_{s}$, and the acceleration of gravity, $g$. That is

$$
\begin{array}{ll} 
& Q=k\left(Q, d, d_{s}, g\right) \text { where } s=m \text { and } b \\
\text { or } \quad K\left(F_{s}, F, \frac{Q s}{Q}\right)=0
\end{array}
$$

It is thus possible to express $F_{s}$ as a function of $F$ depending on the ratio $\frac{Q_{s}}{Q}$ only.

Throughout this discussion, the subscript $m$ attached to a term refers to conditions in the main channel extension, the subscript $b$ refers to conditions in the branch and while there is no subscript used, the term refers to the flow conditions in the main channel.

In this analysis of a dividing flow, a modified "black box " approach is adopted. Initially, the flow in the junction is visualized and the force due to the expanding flow is estimated to act on the fluid. Then the black box is opened to reveal in detail the flow in the junction.

\subsection{Main Channel Extension}

\subsubsection{Momentum Principle}

When a flow $Q_{m}$ occupying a portion of the main channel passes through the junction, it expands to the full width of the main channel extension. There is a variation in the depth of the flow in the junction also. However, this depth 
variation is unimportant as the depths before and after the junction are nearly equal. Due to the expanding width of the flow passage in the junction, a hydrostatic force is developed to act on the fluid.

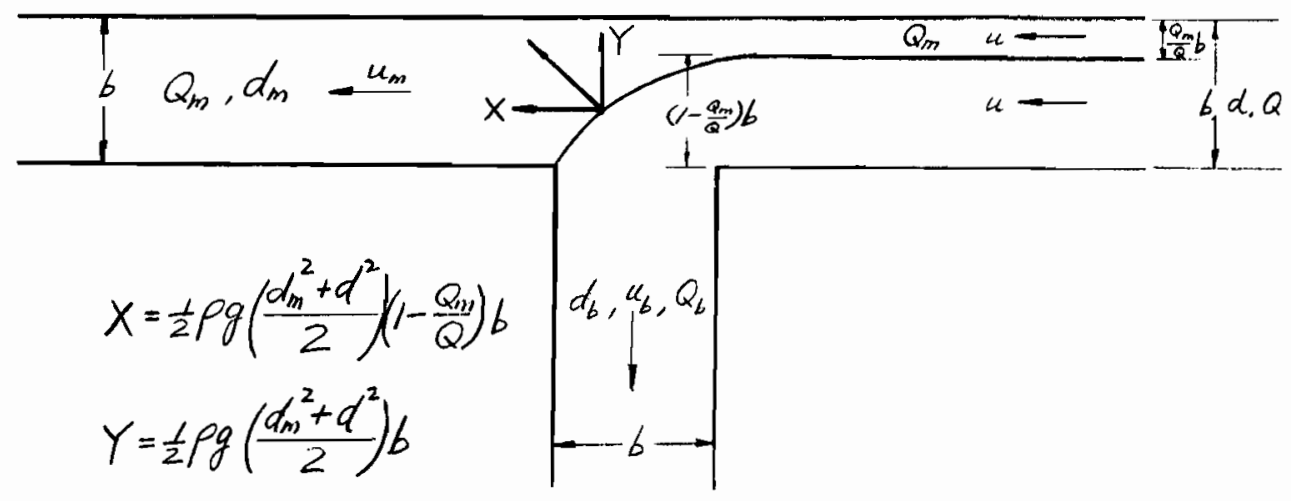

Figure 4.2.1 Force diagram at the junction

It is understood that the dividing streaml ine may end at or near the downstream corner of the junction. In the former case, the hydrostatic force acting on the fluid in the junction is simply that due to the widening flow passage. In the latter case, an additional force is involved. This additional force is small compared with gravity, inertia forces and the force due to the widening flow passage. Furthermore, in the detailed study of the flow in the junction, observations show that one of the streamlines actually ends at the downstream corner of the junction in most cases. Hence in the following analysis, it is assumed that the only force in the junction acting on the fluid is that due to the widening flow passage (Figure 2).

The component of the hydrostatic force acting across the dividing streamline in the direction of the main channel extension is estimated as $\frac{1}{2} \rho g\left(\frac{d_{m}^{2}+d^{2}}{2}\right)\left(1-\frac{Q_{m}}{Q}\right) b$ (Figure 4.2.1). On taking sections before and after the junction, and applying the momentum equation, it can be shown that

$$
\frac{1}{2} \rho g d^{2} b \frac{Q_{m}}{Q}+\rho u^{2} d b \frac{Q_{m}}{Q}+\frac{1}{2} \rho g\left(\frac{d_{m}^{2}+d^{2}}{2}\right)\left(1-\frac{Q_{m}}{Q}\right) b=\frac{1}{2} \rho g d_{m}^{2} b+\rho u_{m}^{2}-d_{m} b
$$


17

This equation is simplified to

$$
\left(1+2 F^{2}\right)\left(\frac{Q_{m}}{Q}\right) d^{2}+\left(\frac{d_{m}^{2}+d^{2}}{2}\right)\left(1-\frac{Q_{m}}{Q}\right)=d_{m}^{2}+2 F_{m}^{2} d_{m}^{2}
$$

where $F$ is the Froude number of the flow def ind by

$$
\text { and } \begin{aligned}
F & =u / \sqrt{g d} \\
\text { and } & F_{m}=u_{m} / \sqrt{g d}
\end{aligned}
$$

Grouping like terms of $d^{2}$ and $d_{m}^{2}$, equation $(4.2)$ is further reduced to

$$
\left(\frac{d_{m}}{d}\right)^{2}=\frac{\frac{1+\frac{Q_{m}}{Q}}{2}+2 \frac{Q_{m}}{Q} F^{2}}{\frac{1+\frac{Q_{m}}{Q}}{2}+2 F_{m}^{2}}
$$

From the continuity equations

$$
\begin{aligned}
& Q=u d b \\
& Q_{m}=u_{m} d_{m}^{b}
\end{aligned}
$$

and

4.4

4.5

it can be shown that

$$
\left(\frac{d_{m}}{d}\right)^{3}=\left(\frac{Q_{m}}{Q}\right)^{2}\left(\frac{F}{F_{m}}\right)^{2}
$$

Substituting equation (4.6) in equation (4.3), it is obtained

$$
\left(\frac{Q_{m}}{Q}\right)^{2}\left(\frac{F}{F_{m}}\right)^{2}=\left[\frac{\frac{1+\frac{Q_{m}}{Q}}{2}+2 \frac{Q_{m_{1}}}{Q} F^{2}}{\frac{1+\frac{Q_{m}}{2}}{2}+2 F_{m}^{2}}\right]^{\frac{3}{2}}
$$

On expanding this equation for small values of $F$ and $F_{m}$, noting that $\frac{Q_{m}}{Q}<1$, it can be shown that

$$
\begin{aligned}
\left(\frac{Q_{m}}{Q}\right)\left(\frac{F}{F_{m}}\right) & \bumpeq 1+\frac{3}{1+\frac{Q_{m}}{Q}}\left(\frac{Q_{m}}{Q} F^{2}-F_{m}^{2}\right) \\
F_{m} & \simeq\left(\frac{Q_{m}}{Q}\right) F
\end{aligned}
$$

or

$4.7 b$ 
To the first approximation, the correction term (last term on the right hand side) of equation $4.7 a$ can be written as

$$
3 F^{2}\left(\frac{Q_{m}}{Q}\right)\left(1-\frac{Q_{m}}{Q}\right)^{2}
$$

The fact that this term is positive reveals that

$$
\left(\frac{Q_{m}}{Q}\right) F>F_{m}
$$

and in a plot of $F_{m}$ aga inst $F$ for a particular value of $Q_{m} / Q$, the slope of the curve will be less than that given by the linear equation $4.7 \mathrm{~b}$ and concavesdown. For a discrepancy of $5 \%$, the linear relationship between $F$ and $F_{m}$ holds for $F<0.4$ for medium range of $Q_{m} / Q$ and holds up to $F=0.9$ for higher values of $Q_{m} / Q$. For all values of $F$, equation 4.7 based on the momentum balance: relates the states of flow in the main channel extension to those in the main channel with the discharge ratio as a parameter.

A comparison of equations 4.6 and $4.7 \mathrm{~b}$ suggests that it is reasonable to assume $d_{m} / d=1$. From the experimental values, it is observed that $d_{m} / d$ ranges from 0.975 for high $F$ to 1.057 for medium $F$. For small values of $F$, $d_{m} / d$ is in between these two limits (Figure 3). Further, it has been shown that to the first order approximation $\left(Q_{m} / Q\right) F>F_{m}$, when $d_{m} / d>1$ from equation 4.6. For all discharge ratios, it has been confirmed experimentally that $d_{m} / d>1$ is the case over most values of $F$. In the next chapter, a comparison of the theoretical and experimental values of $d_{m} / d$ is made. The value of $d_{m} / d=1$ is assumed and it is used in connection with the analys is of the branch flow.

\subsubsection{Energy Principle}

In this section, the principle of the conservation of energy is applied in the analysis of the flow in the main channel extension in a frictionless model. The energy loss due to the formation of hydraulic jumps is neglected. In the next chapter, a more rigorous proof of the energy loss in a hydraulic jump in this channel will be presented. The Bernoulli 's equation relating the conservation of energy upstream 
and downstream of the junction is

$$
\frac{1}{2} \frac{u^{2}}{g}+d=\frac{1}{2} \frac{u_{n}^{2}}{g}+d_{m}
$$

It is simplified to

$$
\frac{d_{m}}{d}=\frac{2+F^{2}}{2+F_{m}^{2}}
$$

Combining equations (4.6) and (4.9), the final form of the equation is

$$
\left(\frac{Q_{m}}{Q}\right)^{2}\left(\frac{F}{F_{m}}\right)^{2}=\left(\frac{2+F^{2}}{2+F_{m}^{2}}\right)^{3}
$$

On expanding the equation (4.10), it can be shown that

$$
\left(\frac{Q_{m}}{Q}\right)\left(\frac{F}{F_{m}}\right) \simeq 1+\frac{3}{4}\left(F^{2}-F_{m}^{2}\right)
$$

When $F$ and $F_{m}$ are both small, clearly, the last term on the right hand side of the above equation can be neglected in a first approximation to give

$$
\left(\frac{Q_{m}}{Q}\right)\left(\frac{F}{F_{m}}\right) \simeq 1
$$

To the first approximation, this correction term in equation 4.10 a can be written as

$$
\frac{3}{4} F^{2}\left[1-\left(\frac{Q_{m}}{Q}\right)^{2}\right]
$$

For $\left(Q_{m} / Q\right) \leqslant 1,\left(Q_{m} / Q\right) F \geqslant F_{m}$ is obtained. This result is similar to that given by the momentum principle. Comparison shows that the correction by the momentum principle is less than 0.7 of that given by the energy princple for all values of $Q_{m} / Q$. This indicates that a better linear approximation is given by the momentum principle. For adiscrepancy of $5 \%$, the linear relationship given by the energy principle holds for $F<0.3$ when $Q_{m} / Q<0.5$, and to $F=0.6$ for higher values of $Q_{m} / Q$. It is clear that for small values of $F$ and $F_{m}$, no energy loss is encountered. In reality, when $F$ and $F_{m}$ are small, no hydraulic jumps are anticipated. 


\subsection{Branch Channel}

\subsubsection{Momentum Principle}

The flow in the branch channel is complicated. Reverse flows can extend as far as the exit end of the branch channel. Hydraulic jumps are also formed when the inflow is fast enough. In some cases, both reverse flows and hydraulic jumps are observed in this channel (Figure 4.3.1).

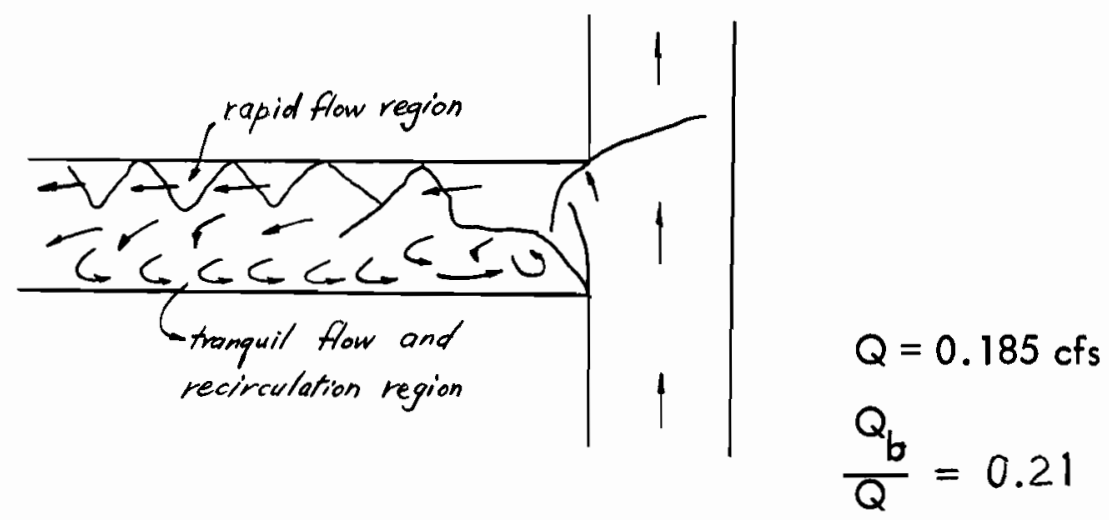

Fig. 4.3.1 Complex flow in branch channel

A simple way to attack the problem of the branch flow is from the momentum point of view. The force which deflects part of the inflow into the branch is the hydrostatic force acting on the branch flow at the junction. This force is the reaction of the force which acts on the flow passing into the ma in channel extension. The component of this hydrostatic force acting in the direction of the branch channel can be estimated as $\frac{1}{2} \rho g\left(\frac{d_{n}^{2}+d^{2}}{2}\right) b$ ('Refer to Figure 4.2.1)

Since there is a more extensive recirculation region in this branch, it is: necessary here to include a width contraction coefficient, $c$. This contraction coefficient is the ratio of the active conveying width of the channel to the actual width of the channel, b. The recirculation regian which occupies part of the channel width contributes no net momentum flux or mass flux in a one-dimensional flow model. Thus, when no reverse flow is being considered at low Froude numbers $F, c$ is simply equal to one.

Irrespective of the complexity of the flow in the branch channel, the mean depth of the flow is taken as the depth along the centre line of the channel. The mean velocity is $u_{b}$, given by the continuity equation

$$
Q_{b}=u_{b} d_{b} b
$$


The mean Froude number in the branch channel, $F_{b}$ ' is expressed as

$$
F_{b}=u_{b} / \sqrt{g d_{b}}
$$

From continuity equations,

$$
Q_{b}=u_{b} d_{b} b=u_{b}^{\prime} d_{b}(c b)
$$

where $u_{b}^{\prime}$ is the mean velocity in the active flow region and "cb" is the active width of the branch. It is clear that

$$
u_{b}=c u_{b}^{\prime}
$$

Substituting this value of $u_{b}$, equation 4.13 becomes

$$
F_{b}=\frac{c u_{b}^{\prime}}{\sqrt{g d b}}
$$

The momentum equation for the branch flow is given by

$$
\frac{1}{2} \rho g\left(\frac{d_{m}^{2}+d^{2}}{2}\right) b=\frac{1}{2} \rho g d_{b}^{2} b+\rho u_{b}^{\prime 2} d_{b}(c b)
$$

From the continuity equations,

$$
\begin{aligned}
Q_{b} & =u_{b}^{\prime} d_{b}(c b) \\
\text { and } \quad Q & =u d b
\end{aligned}
$$

the velocity ratio $u_{b}^{\prime} / u$ can be expressed as

$$
\frac{u_{b}^{\prime}}{u}=\frac{Q_{b}}{Q} \cdot \frac{d}{d_{b}} \cdot \frac{1}{c}
$$

Substituting $u_{b}^{\prime} / u$ in equation 4.17 gives the equation

$$
1+\left(\frac{d_{m}}{d}\right)^{2}=2\left(\frac{d_{b}}{d}\right)^{2}+4\left(\frac{Q_{b}}{Q}\right)^{2} F^{2} / c\left(\frac{d_{b}}{d}\right)
$$


For $d_{m} / d=1$ (section 4.2.1), equation (4.19) can be rearranged to obtain a unique relationsh ip between the discharge ratio, $Q_{b} / Q$, and the depth ratio of the branch to the main channel, $d_{b} / d$. The parameter used is the Froude number in the main channel, $F$.

$$
\begin{array}{ll} 
& \left(\frac{Q_{b}}{Q}\right)^{2}=c \frac{d_{b}}{d}\left[1-\left(\frac{d_{b}}{d}\right)^{2}\right] / 2 F^{2} \\
\text { or } & \left(\frac{d_{b}}{d}\right)-\left(\frac{d_{b}}{d}\right)^{3}=2 F^{2}\left(\frac{Q_{b}}{Q}\right)^{2} / C
\end{array}
$$

A different grouping of the variables in equation (4.17) will yield a relationship relating the Froude number of the flow in the branch to that in the main channel with the discharge ratio as a parameter.

$$
F=F_{b} /\left[\left(\frac{Q_{b}}{Q}\right)\left(1+\frac{2}{c} F_{b}^{2}\right)^{\frac{3}{4}}\right]
$$

\subsubsection{Energy Principle}

In this section, an attempt is made to relate the flow in the branch to the main channel by applying the energy principle. The hydraulic jumps, whenever they are formed in the branch, are assumed to be of the order of those formed in the main channel extension. As stated in section 4.2.2, the energy loss due to these jumps is negligible. Other losses such as frictional loss are neglected.

Bernoulli's equation relating the flow, which is being diverted to the branch, upstream of the junction and in the branch is

$$
\frac{1}{2} \frac{u^{2}}{g}+d=\frac{1}{2} \frac{u_{b}^{\prime}}{g}+d_{b}
$$

where $u$ is the mean velocity of the flow in the main channel; $u_{b}^{\prime}$ is the velocity in the active conveying width of the branch channel given by $u_{b}^{\prime}=u_{b} / c$ (equation 4.15); $u_{b}$ is the mean velocity of the flow in the branch; $d$ and $d_{b}$ 
are the mean depth of the flow in the main channel and in the branch respectively and $g$ is the acceleration of gravity. Rearranging the terms and combining with the continuity equations, equation $(4.23)$ is finally put in the form

$$
\left(\frac{Q_{b}}{Q}\right)^{2}\left(\frac{F}{F_{b}}\right)^{2}=\left(\frac{2+F^{2}}{2+\frac{F_{b}^{2}}{C^{2}}}\right)^{3}
$$

where $F=\frac{u}{\sqrt{g d}}$ and $F=\frac{u_{b}}{\sqrt{g d}}$ are the Froude numbers of the flows in the main channel and in the branch respectively.

\subsection{Comparison of Theory with Experiment}

In this section, some sample cases of the theory are compared with the experiment. In the next chapter, a more detailed comparison between the theory and the experiment will be given.

For the flow in the main channel extension, both the momentum and the energy principles reveal that there is a linear relationship existing between small values of $F_{m}$ and $F$ depending only on the discharge ratio $Q_{m} / Q$. In Figure 4 , the above relationship is shown as a line of $45^{\circ}$ in the plot of $\Delta F / / \Delta F$ against $Q_{m} / Q$. The mean slope of the experimental curves covering the largest possible range of $F$ for different values of $Q_{m} / Q$ are measured (from Figure $11 \mathrm{la}$ ) and are plotted on the same Figure 4. It is seen that this linear relationship holds when the discharge ratios are either close to zero or one. For intermediate values of $Q_{m} / Q$, there is a nearly constant deviation between this simple theoretical approximation and the experiments. A higher theoretical value of $F_{m}$ is obtained for a given discharge ratio and a specified Froude number. This discrepancy is owing to the approximation $F_{m}=\left(Q_{m} / Q\right) F$. It has been shown previously that to the first order approximation $\left(Q_{m} / Q\right) F>F_{m}$ is obtained. The use of this inequality results in a better prediction of the flow.

For the flow in the main channel extension, equations 4.7 and 4.10 are evaluated and tabulated in Table II for all values of $F$ from zero to 0.8 for values of $Q_{m} / Q=0.90,0.65$, and 0.25 . The results are plotted with the experimental results in Figure 5. The values given by the momentum and energy principles agree with each other. This fact indicates that the estimated hydrostatic 
force in the junction used in the momentum balance is of the right magnitude in this frictionless model. Theoretical predictions are good except at high Froude numbers.

For the flow in the branch, equation 4.22 based on the momentum principle has been evaluated for $Q_{b} / Q=0.90,0.65$, and 0.25 . The results are plotted in Figure 6 and are compared with the experimental results. Good agreement between the theory and the experiment is shown except at high Froude numbers $F$. In the evaluation of the branch flow in this chapter, the tabulated values of $c$ in Table III are used. The choice of $c$ will be given in section 5.4. Similarly, the theoretical results from the momentum equation 4.21 with and without $(c=1)$ the application of contraction coefficients are shown in Figure 7. The experimental curves are also shown for comparison. In this plot of $Q_{b} / Q$ against $d_{b} / d$ with $F$ as a parameter, the importance in the proper choice of the contraction coefficient is demonstrated.

The limits of the F-curve in Figure 7 corresponding to free-overfall condition* in the branch have been obtained theoretically by solving the momentum equation 4.22 in which $F_{b}=0.7$ is used. It has been found in this work that under free-overfall condition in the branch, $F_{b}$ is nearly equal to 0.7 for $Q_{b} / Q>0.5$. The theoretical limiting points show satisfactory agreement with the experimental limiting points.

When equation 4.24 based on the energy principle is evaluated, it is found that poor prediction is given for the flow in the branch. This is due mainly to the irreversible process of the flow on turning the corner into the branch. It is noted here that due to flow separation on the corner, the flow is rapid in the entrance region to the branch. Then the flow expands into a wider region further downstream. It is difficult to visualize the flow conditions in this entrance region.

* Free-overfall condition is not actually attained in this model. At the exit end of each channel, there is a $\frac{1}{4}$-inch rubber strip sticking up from the bed of the channel for sealing purpose. Hereafter, for convenience, the term "free-overfall condition " is used when the gate is lowered below the bed elevation in this model. 
No simple theory is sufficiently accurate to describe the flow in the branch without the step by step analysis of the flow in this region. No further comment is made of the branch flow based on the energy principle.

\subsection{Similarity of the Model to a Simple Straight Channel}

It has been found in this work that the flow in the main channel extension behaves in a general way like that in a straight channel even when there is flow in the branch. In the branch, there is a greater discrepancy.

To investigate the similarity of a dividing flow to that of a simple straight channel in terms of Froude numbers, separate tests have been run with the branch completely blocked off by a block of wood at the junction. Different total discharges were fed into the main channel while the sliding gate at the end of the main channel extension was varied. The results in terms of Froude number $F$ were plotted against the ratio $w / d$ where $w$ is the height of the gate above the bottom of the channel and $d$ is the mean depth of flow at 24 inches upstream of the gate (Figure 8).

A theoretical curve in terms of $F$ and $w / d$ can be obtained from an elementary discharge equation. In 1717, Poleni $^{(4)}$ gave an elementary discharge equation for two-dimensional flow over a vertical sharp-crested weir at the end of a horizontal channel as

$$
\begin{aligned}
& q=\frac{2}{3} C_{d} \sqrt{2 g} h_{e}^{\frac{3}{2}} \\
& C_{d}=0.608+0.334\left(\frac{h_{t}}{h_{\epsilon}+w}\right)^{2}
\end{aligned}
$$

with as given by Bazin. In the above equations, $q$ is the discharge per unit width of the channel, $C_{d}$ is the discharge coefficient, $h_{e}$ is the head on the weir and $w$ is the height of the weir. The results from evaluating equation 4.25 are plotted in Figure 8 for comparison. It is noted that a higher Froude number is given by the straight channel used in this work for all values of $w / d$. This straight channel is of small scale. Consequently, the boundary effects are important resulting in an increase of the Froude numbers. 
In Figures 9 and 10, the experimental results in this work of the main channel extension and the branch are compared with that in a straight channel.

For the main channel extension, the similarity of the flow to a straight channel is good (Fig. 9). For the branch flow, there is good agreement with a straight channel for both high and very low discharge ratios, say less than 0.1 . For small discharge ratios, a higher Froude number than that in a straight channel is obtained for the same $w / d$ ratio. This is due to the non-uniformity of the flow. In these cases, the recirculation regions and the boundary conditions are important. The recirculation regions in the main channel extension are less extensive and the increase in Froude number for the same $w / d$ is less significant. On the whole, the similarity of the flow in either channel to a straight channel is satisfactory. 


\section{EXPERIMENTAL RESULTS AND DISCUSSION}

\subsection{Limiting Cases}

Once the similarity of the flow between the main channel extension or the branch to a straight channel is established, it is possible to discuss the limit of the performance of each channel. It is apparent that the simple theory presented in the last chapter is not limited in its application only to tranquil flow as the equation can also be solved for $F, F_{m}$ and $F_{b}$ greater than one. Since the inflow and outflow are both tranquil in this model study, the following investigation is intended to fix the limits of the Froude numbers for tranquil flow only.

The limiting Froude numbers obtained in this model (for both the geometry and a discharge ratio of unity in a channel) are

$\begin{array}{lll} & F & \mathrm{w} / \mathrm{d} \\ \text { Straight channel model } & 0.76 & 0.14 \\ \text { Main channel extension } & 0.78 & 0.15 \\ \text { Branch channel } & 0.68 & 0.22\end{array}$

The Froude number limit for the branch is lower than that given by the straight channel. The former limit, however, occurs at a higher $w / d$ ratio. Since there is good similarity of the branch flow to the flow in a straight channel, it suggests that if the geometry such as the gate elevation, can be adjusted to obtain the same $w / d$ ratio, the same Froude number limits can be obtained.

By extrapolating the curves in Figures 9 and 10 to $\mathrm{w} / \mathrm{d}=0$ (that is, true free-overfall condition), it is found that a Froude number limit of about 0.95 is reached in all cases. The important factor controlling the limit in th is model is the ratio $w / d$. The presence of the recirculation region in the main channel extension and in the branch for small discharge ratios affects both the depth and the velocity of approach of the flow even for the same geometrical setting of the gates as in the straight channel. Owing to the non-uniformity of the flow, higher Froude numbers in the main channel extension and in the branch than that in a straight channel are obtained. However, owing to the similarity of the flow in the main channel extension and the 
branch to a straight channel, it is believed that the same Froude number limit is reached under true free-overfall conditions. Further increase in the inflow will change little of this limit for a fixed geometry of the model.

There are important engineering applications of the limiting Froude numbers corresponding to different gate positions in a channel. These limits can be found either experimentally or theoretically by examining the typical curves as shown in Figures 9 and 10. The fact that similarity of the flow in the several channels of this model to a straight channel holds provides a valuable and simple method to calculate the Froude number of the flow in a channel once the gate elevation is known. This method is good for a discharge ratio in a channel equal to one or for very low gate elevations. For small discharge ratios in the main channel extension or in the branch, however, lower Froude numbers are predicted by the simple straight channel owing to the non-uniformity of the flow.

\section{2 Overall Performance of the Main Channel Extension}

In this section, a comparison of the theory and the experiment on the flow in the main channel extension is made. The experimental results and the theoretical results are presented in Figures 1 la and $b$ respectively. Curves of constant discharge ratios shown in Figures lla are sketched as described in Section 3.2. The theoretical curves with their limits are plotted by evaluating equation 4.10 . A $45^{\circ}$ line is drawn in each figure to correspond to a discharge ratio of one in this channel (or in a straight channel) in a frictionless model.

For simplicity, three simple curves having discharge ratios, $Q_{m} / Q$, of $0.9,0.65$, and 0.25 are shown in Figure 5 to compare with the theoretical predictions using both the momentum and the energy principles. It is observed that the theoretical predictions are most satisfactory for $F<0.4$. At high Froude numbers, both the momentum and the energy theory are inaccurate. In these cases, the flow at the junction is complicated. For the momentum theory, the hydrostatic force which is estimated to act on the dividing streamline is no longer valid. The possible source of error in the energy conservation theory is from the assumption of negligible energy loss. The flows at the junction are changing from a tranquil flow to a rapid flow. In a simple theory, 
where the effect of changing state of the flow at the junction has not been accounted for, this discrepancy is not surprising. In the following section, an attempt is made to evaluate the energy loss due to the presence of hydraulic jumps.

The theoretical limits of these F - curves are shown in Figure $11 \mathrm{~b}$. These limits are obtained simply by transposing the theoretical limits of the flow in the branch channel (Figure 7). Discrepancy of the limits between the theory and the experiment rests on the use of the assumed limit of $F_{b}=0.7$ for all values of $Q_{b} / Q$ (Section 4.4).

It is found both experimentally and theoretically that the limits of $F$ at low discharge ratios are well below the maximum limit of $F=0.85$ when $Q_{m} / Q$ is high, for example, $F$ limit $=0.50$ for $Q_{m} / Q=0.10$. This is in accord with the physical nature of the flow. When the gates are adjusted to reduce the flow into the main channel extension either by raising or lowering the gate at the exit ends of the main channel extension or the branch respectively, the depth of flow in the main channel is increased rendering a decrease in the values of $F$. Thus, a decrease in $Q_{m} / Q$ induces a decrease in $F$ as a consequence.

In Chapter 2, it has been stated that reverse flows occur in the main channel extension as a result of flow separation at the junction when discharge ratios $Q_{m} / Q$ are small. But Figure 5 reveals that the theory predicts the flow extremely well for small discharge ratios. Thus, it is apparent that reverse flows or the recirculation regions have little effect on the overall performance of the main channel extension.

This recirculation region from flow separation to reattachment is limited to about three times the channel width (Plate 2). The depth measurements in this channel are taken at 34 inches from the centre of the junction, beyond the point of reattachment. It is known that the discharge in a channel regulated by a sliding gate depends primarily on the conditions of depth and velocity close to the weir and not on the condition far upstream of the weir. Near the gate, the region is free from reverse flows. Hence the determination of the Froude number in this channel is independent of reverse flows. 


\subsubsection{Energy Loss}

To evaluate the energy loss in the section between the main channel and the main channel extension, the following approach is adopted. Neglecting the effect of wall friction, the main source of energy loss is due to the formation of hydraulic jumps. A further assumption made is that the hydraul ic jump is assumed to form at the inlet section of the main channel extension just after the flow passes through the junction. With reference to Figure 5.2.1, the flow in the reach between sections $U$ and $J$, the

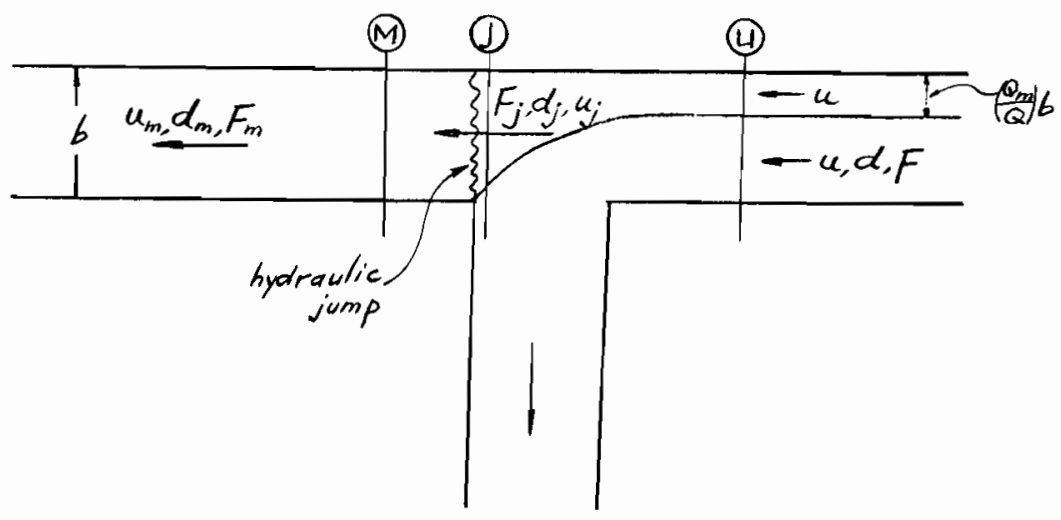

Figure 5.2.1

sections upstream of the junction and before the jump respectively, can be related by equating the total energy head, since no energy loss is assumed here on the flow expansion

$$
\frac{1}{2} \frac{u^{2}}{g}+d=\frac{1}{2} \frac{u_{i}^{2}}{g}+d_{i}
$$

The continuity equation is

$$
\operatorname{ud}\left(Q_{m} / Q\right) b=u_{i} d_{i} b
$$

where subscript $i$ refers to the flow in the junction just before the jump. Evaluating these two equations, it can be found that

$$
\frac{d_{j}}{d}=\frac{2+F^{2}}{2+F_{j}^{2}}
$$




$$
\text { or }\left(\frac{Q_{m}}{Q}\right)^{2}\left(\frac{F}{F_{i}}\right)^{2}=\left(\frac{2+F^{2}}{2+F_{i}^{2}}\right)^{3}
$$

These equations relate the flow conditions expressed in terms of Froude numbers, upstream of the junction and in the junction before the jump, depending on the flow distribution.

Similarly, on taking sections $J$ and $M$ of the flow before the jump and after the jump respectively, Figure 5.2.1, the momentum equation can be written as

$$
\frac{g d_{i}^{2}}{2}-\frac{g d_{m}^{2}}{2}=d_{m} u_{m}^{2}-d_{i} u_{i}^{2}
$$

and the continuity equation as

$$
u_{i} d_{i}=u_{m}^{d}
$$

where the subscript $m$ refers to a quantity of the flow after the jump in the main channel extension. In the presence of hydraulic jumps,

$$
F_{i}^{2}=\frac{1}{2} \frac{d_{m}}{d_{i}}\left(1+\frac{d_{m}}{d_{i}}\right)
$$

where $F_{i}=u_{i} / \sqrt{g^{d}}$ is the Froude number of the flow in the junction just before the jump.

The energy loss $\Delta H$ across the jump can be written as

$$
\Delta H=\left(d_{i}-d_{m}\right)+\frac{1}{2 g}\left(u_{i}^{2}-u_{m}^{2}\right)
$$

Combining equations (5.7) and (5.8), the energy loss can be written as 


$$
\frac{\Delta H}{d_{i}}=-\frac{1}{4} \frac{d_{i}}{d_{m}}\left(1-\frac{d_{m}}{d_{i}}\right)^{3}
$$

where in the case of a hydraulic jump, $d_{m} / d_{i}$ defined by equation (5.9) is always greater than one. Some typical results are tabulated below. When $Q_{m} / Q=0.9$, $F_{i}$ and $d_{m} / d_{i}$ have been evaluated from equations $(5.4)$ and $(5.7)$ respectively, for two values of $F$, corresponding to the cases where hydraulic jumps formation is possible. The values of $d_{m} / d$ and $\Delta H / d_{i}$ are also calculated.

\begin{tabular}{|l||c|c|c|c|}
\hline$Q_{m} / Q=0.9$ & $F_{i}$ & $d_{m} / d_{i}$ & $d_{m} / d$ & $H / d_{i}$ \\
\hline$F=0.8$ & 1.54 & 1.73 & 1.04 & 0.06 \\
\hline$F=0.7$ & 1.62 & 1.84 & 0.99 & 0.08 \\
\hline
\end{tabular}

The results reveal that the energy loss when hydraulic jumps appear cannot be neglected. The results based on this modified theory are plotted in Figure 5 to compare with those of the simple theory. The effect of taking into account the energy loss on shifting the theoretical curves to simulate the experimental curves is demonstrated.

In this crude model, no provision is made for adjustment to correspond to varying flow conditions as the discharge ratios vary. This crude model is not satisfactory. However, it helps to point out the fact that at high Froude numbers, the energy loss is no longer negligible.

The improvement of the theoretical results due to more precise and elaborate analys is is slight since the changing flow conditions at high Froude numbers are not fully understood. The labour involved is unwarrented. Thus, the simple theory presented in Section 4.2.2 in describing the flow in the main channel extension is sufficient for general application.

\section{3 Overall Performance of the Branch Channel}

In this section, the experimental results of the branch channel are presented and the performance of the branch channel is discussed. In Figure $12 a$, the curves of 
constant discharge ratios are sketched as described in Section 3.2. A $45^{\circ}$ line is drawn to correspond the flow in a straight channel.

It is shown experimentally that for small discharge ratios of $Q_{b} / Q$, the curves all terminate at a constant $F$ of about 0.8 and curl up. This fact suggests that the flow on turning the corner to the branch becomes critical either in the junction or in the branch. Thus, by varying the parameters such as the depths and the discharge distributions in the channels, different Froude numbers in the branch can be obtained without affecting the state of flow in the main channel. These conditions prevail in cases only when the approaching Froude number of the flow in the main channel, F, is high and near the limiting value.

Examples of these cases are shown in Figures 21 and 22. For $F=0.68$, there is a critical section forming across the inlet section of the branch (Figure 21). For $F=0.77$, Figure 22 reveals that the flow in the junction becomes rapid. Figure 20 shows that no such critical section: $(s)$ is (are) formed when the Froude number $F$ is small even though the discharge ratio $Q_{b} / Q$ is high. The flow conditions as shown in Figures 20, 21 and 22 are correlated in Figure 12a. The positions of these points in the experimental curves confirm that rapid flow in the junction can be found only when the approaching flow is relatively fast, that is, $F$ is relatively high.

Theoretically, similar curves as shown in Figure $12 a$ can be generated with the momentum principle (Figure 12b). However, the success of a theoretical analys is of the branch flow depends very much on the choice of the contraction coefficients. It is intended in the next section to present in detail the method which is used in choosing the contraction coefficients as tabulated in Table III. The presentation of the theoretical results and discussion will then follow.

\subsection{Contraction Coefficients}

When equations (4.21) and (4.22) are evaluated with $c=1$ (assuming the effect of reverse flow is unimportant), and the results are plotted, it is found that there is wide discrepancy between the theory and the experiment. To simulate better the theory to experimental results, a contraction coefficient, $c$, is applied to account for the presence of extensive recirculation regions or reverse flows in both downstream and 
cross-channel directions (Figure 4.4). The contraction coefficient is the ratio of the active width of delivery in the branch to the actual width of the channel. To evaluate

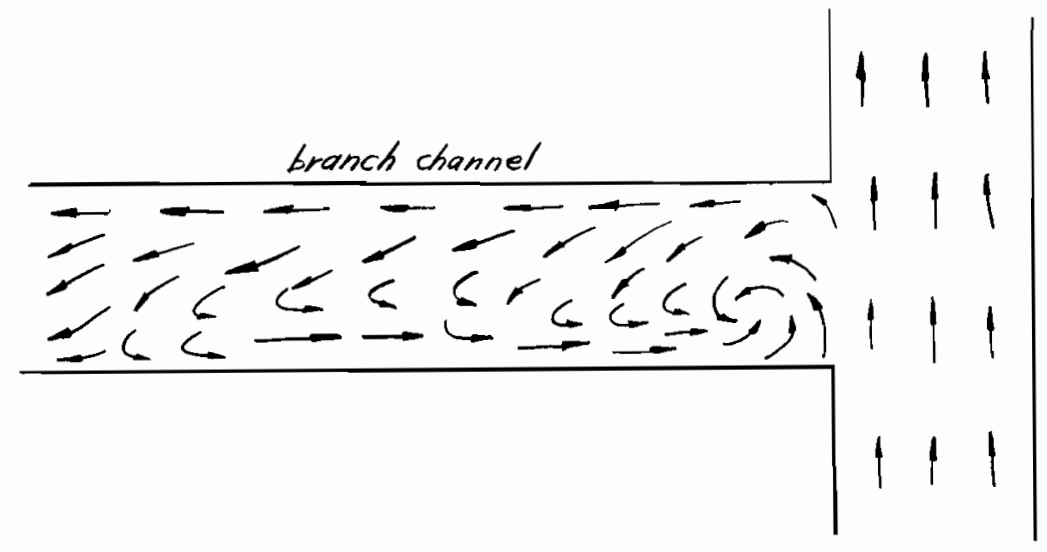

Figure 4.4 Reverse flow in the branch channel

equations (4.21) and (4.22), the value of $c$ is chosen by visual observations of the flow. Since it is the reverse flow in a recirculation region which can be detected visually, the actual width of the whole recirculation region cannot be determined directly. Bearing in mind that the width of the reverse flow region is not clearly defined and that final adjustment has to be made for various flow parameters such as depth ratios and Froude numbers, the width of the recirculation region is taken as twice the width of the reverse flow region.

When the flow is deep, or when the Froude number of the flow in the main channel is low, say less than 0.5 , there is no extensive reverse flow apparent in the branch. This is true for all discharge ratios. In these cases, the contraction coefficient is evidently one. In cases of higher Froude number in the main channel, say greater than 0.5, the extent of the reverse flow regions in the branch varies. As the discharge ratios become smaller, the recirculation region in the branch extends both lengthwise and across the channel. Evidently, when there is no delivery in the branch, the flow there is wholly recirculating. The contraction coefficient, $c$, in this case is zero.

After examining the growth of the recirculation region from the sketches made in each test, several trials in the choice of $c$ are made. Finally, a step-wise variation of the contraction coefficient is adopted for various ranges af discharge ratios, 
and Froude numbers $F$. The results are tabulated in Table III. When th is choice of $c$ is used to evaluate equations 4.21 and 4.22 , the theoretical curves show marked improvement in their ability to simulate the experimental curves (Figure 7).

It is noted that several regions in the plot of the theoretical results overlap each other owing to the step-wise variation of $c$. In Figure 12b, overlapping regions in the theoretical curves are adjusted to spread the curves more evenly. These adjusted curves are marked accordingly in the figure. Sections of the theoretical curves extending beyond the limiting Froude numbers are also cut off.

A comparison of the experimental results (Figure 12a) and theoretical results (Figure $12 b$ ) reveals that agreement between the two is satisfactory. The same pattern is followed by the two sets of curves. Indeed, the tendency of the theoretical curves for small discharge ratios on the limiting value of $F$ can be interpreted as a fair approximation of the experimental curves. The latter curves are themselves a fair representation of the experimental results. The model used in this theoretical analysis is good provided a proper choice of the contraction coefficient is made.

Alternatively, as suggested by Taylor and Grace $(9,3)$, it is advantageous to present the data in terms of ratios of depthand discharge, that is, $d_{b} / d$ and $Q_{b} / Q$ respectively. With a few desirable modifications to the ir methods, the experimental results are plotted in Figure 13a while the theoretical curves based on equation 4.21 , with and without the application of contraction coefficients, are plotted in Figure $13 \mathrm{~b}$ for comparison.

It is noted that the theory predicts the flow sat isfactorily for small values of $F$ where no effect of the recirculation region on the flow has to be accounted for. At higher Froude numbers, it is seen (Figure 13b) that the curves without the application of the contraction coefficient do not follow the general pattern. After the application of an appropriate contraction coefficient, the theoretical results are much improved to simulate the experimental results. This contraction coefficient, however, cannot account for all the discrepancies between the theoretical and the experimental results.

In Figure 13b, the end points of the theoretical curves are computed by putting $F_{b}=0.70$ in equation 4.22 . It has been realized from the prel iminary study 
that the locus of the end points of these F-curves represents the free-overfall conditions that corresponds closely to the limiting Froude number in the branch, $F_{b^{\prime}}$ of 0.70

(Figures 14 and 15). For particular values of $F$, after substituting $F_{b}=0.70$ in equation 4.22 and choosing an appropriate contraction coefficient from Table III, the end points of the curves in Figure $13 \mathrm{~b}$ can be determined. These results show good agreement with the experimental results of Grace ${ }^{(3)}$.

\subsection{General Review}

There are many occasions in which the problem of a dividing flow in an open channel will be encountered. The frequent applications are in flood routing, dra inage, sewage treatment, and irrigation. For the engineer, the general behaviour of the dividing flow problem is important. The experimental results presented here will enable the engineer to grasp the general nature of the problem. The theoretical approach also shows some feasibility of attacking the problem from the mathematical point of view. For each practical situation, a model test is recommended to aid visualization of the change in local conditions. The simple theory is particularly useful in cases when the Froude number, $F$, is very small, say less then 0.4 .

A dividing flow in a model in which the angle of intersection and the width of the branch could be varied has been studied by Grace ${ }^{(3)}$. In each case, his results indicated that the loci of the limits under free-overfall conditions were different but the same general pattern was followed. In Chapter 7 , the momentum theory presented above will be extended and correlated with Grace's experimental results. This analysis further suggests that a model test for each individual dividing flow problem is recommended. More detail will be given in Chapter 7 . 


\section{DETAILED STUDIES}

To enable gereralization of both the experimental and theoretical approaches, a good understanding of the details and local phenomena is essential. In th is chapter, a short description is presented to the development of reverse flows, hydraulic jumps and non-unifrom flows in the junction. Surface Discontinuity at the upstream corner of the junction is also described.

\subsection{Reverse Flow}

A phenomenon in the study of dividing flow that deserves particular attention is the extensive recirculation region in the branch channel or the relatively weaker eddying region that appears in the main channel extension. The reverse flow in an eddying region presents the most striking feature in a channel flow since its direction is opposite to the general flow. There is no clear indication of the position of the streamline separating the recirculation region from the general flow. Hence, the term "reverse flow" is used in lieu of "eddying region " or "recirculation region" whenever it is more appropriate.

To describe the development of the recirculation region, it is noted that reverse flow is the consequence of flow separation. In the branch channel, when the recirculation region grows, it sometimes extends lengthwise to the exit end of the branch and corsswise to more than half the width of the channel (Plate 3). In the ma in channel extension, the recirculation region has a smaller scale generally, ending at a distance of three times its width downstream and limited in width to less than one-third of the channel (Plate 2). In both channels, when the discharge ratios are very small, large recirculation regions extending across the respective channels are formed. The "when" and "how" that govern the growth of a recirculation region had not been obtained theoretically or experimentally.

A series of observations were run on each channel separately. The discharge ratio was reduced step by step to zero. In Figures $16 \mathrm{a}$ to $\mathrm{g}$, the sketches show the development of the recirculation region. Similarly, Figures $17 \mathrm{a}$ to $\mathrm{h}$ show the growth of the recirculation region in the main channel extension. 


\subsection{Hydraulic Jump}

To understand the development of the hydraulic jumps for different discharge ratios, some particulars of the flow distribution and its regulation must be borne in mind. It must be realized that for free-overfall conditions in both the main channel extension and the branch, the discharge in the branch is about $20 \%$ of the total discharge. Hence, to get $Q_{b} / Q$ less than 0.2 , or to get more than 80 percent of the total discharge into the main channel extension, the flow into the branch has to be reduced. This can be done only by raising the gate at the exit end of the branch.

When hydraulic jumps are formed in the branch channel, they appear, in generally, as criss-cross waves occupying about half the channel width while the other half is occupied by the recirculation or slow flow region. The hydraulic jumps in the branch extend to about half the length of the channel.

When the Froude number of the flow in the main channel is greater than 0.7 , the flow in the junction can become critical and hydraulic jumps are initiated in the main channel extension. Initially the disturbance in the main channel extension just downstream of the junction appears as a "bow wave" which is caused by the streamlines deflecting from the downstream corner of the junction. The flow in the junction may acquire a higher velocity or a shal lower depth as the downstream cond itions vary, such as an increase in the discharge ratio of the main channel extension. As the flow in the junction becomes faster, or the corresponding Froude number becomes bigger, undular jumps appear in the main channel extension. These jumps extend far into the downstream direction and die out gradually on approaching the exit end of the channel. The term "standing waves" has been used in related studies by contemporary writers to signify these undular jumps. The effect of these "standing waves" on a dividing flow has been emphasized in the report by Grace ${ }^{(3)}$. The development from "bow waves " to undular jumps corresponds to an increese in the discharge ratio, $Q_{m} / Q$, of the main channel extension. When $Q_{m} / Q=0.8$, the hydraulic jumps will develop from undular jumps to a weak jump which corresponds to a further increase of the Froude number of the flow in the junction. The classification of the different types of hydraulic jumps used here is that adopted by the U.S. Bureau of Reclamation (1). When a weak jump is formed, the standing waves in the reach of the main channel extension are damped out leaving only 
a single jump just downstream of the junction. (Figure 6.2).

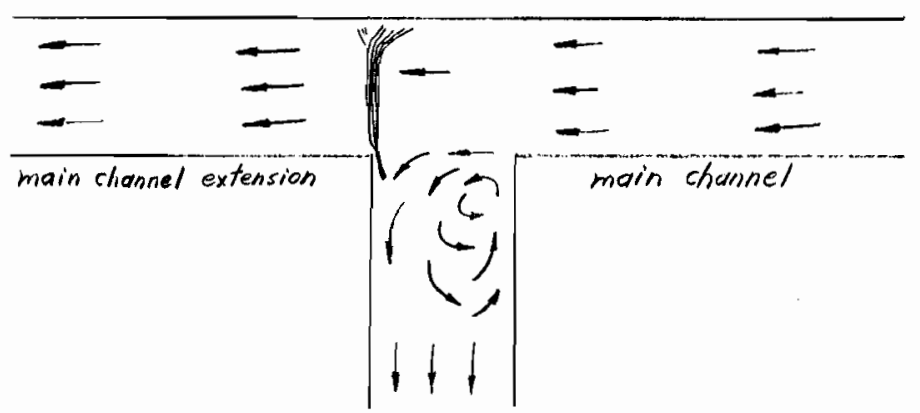

Figure 6.2 Weak jump in the junction

On further increase of $Q_{m} / Q$ by adjusting (raising) the gate at the exit end of the branch channel, the standing wave profile reappears owing to a decrease in Froude numbers of the flow in the junction. This decrease in Froude number corresponds to an increase in depth or a decrease in velocity of the flow in the junction as a consequence of the decreasing discharge into the branch channel.

Further decrease of $Q_{b} / Q$ to and below 0.1 will reduce the flow in the branch to a sluggish circulation (Figure $16 \mathrm{~g}$ ). In this range of flow, the slowly circulating flow in the branch has little effect in modifying the flow condition in the straight channel of this model. Consequently, the flow in a straight channel is simulated. Since the flow in the straight channel is subcritical, no hydraulic jump is expected for flows in this range.

In Figure 19, the formation of hydraulic jumps (or standing waves) in the main channel extension is illustrated as a function of the ratio of amplitude to depth and the discharge ratio. The amplitudes of the first crest downstream of the junction and of the periodic waves (standing waves) at 34 inches from the centre of the junction are drawn. These plots illustrate the development of undular jumps to weak jumps as a function of discharge ratios $Q_{m} / Q$. It has been noted in this experiment that under different discharge ratios, $Q_{m} / Q$, the standing waves have a wave-length ranging from $3 \frac{1}{2}$ inches to 6 inches. It is believed that the wave-length is also a function of the width of the channel. 


\subsection{Flow in the Junction}

The flow in the junction has been studied for three cases. In these cases, the inflow Froude numbers in the main channel, $F$, are $0.28,0.68$ and 0.77 . The contours of constant Froude number of the flow in the junction are plotted for each case.

When $F$ is low, 0.28 in this case, the local flow Froude number in and around the junction has a maximum of 0.47 and a minimum of 0.15 (Figure 20). The surface in and around the junction is smooth and no bow waves or hydraulic jumps are found. The discharge ratio of the flow in the main channel extension is small (only 28\%) and the flow separates from the wall at the junction (Plates 2 and 6 ).

For $F=0.68$, the flow on turning the corner to the branch becomes critical locally. The $\mathrm{pl}$ of in Figure 21 shows that a rapid flow region extends across the entrance to the branch from the upstream corner of the junction. For this relatively high inflow Froude number a bow wave is formed near the downstream corner of the junction (Figure 21 and Plate 7). The discharge ratio of the main channel extension is 0.5 and a relatively small recirculation region can still be observed in the main channel extension.

On increasing gradually the inflow Froude number and the discharge ratio of the flow in the main channel extension, the rapid flow region begins to grow in length and spread out in the clockwise direction (Compare Figures 21 and 22). Finally, the flow in the whole junction region becomes supercritical (Figure 22). In Figure 22 (and Plates 8 and 9) it has been shown that when $F=0.77$ and $Q_{m} / Q=0.69$, hydraulic jumps are found in lieu of bow waves since the flow in the junction is supercritical. Maximum Froude number observed near the entrance to the branch channel is 2.1. There is a region of rough water occupying half of the channel width in the inlet section of the branch. The other half is occupied by a recirculation region due to flow separation at the upstream corner of the junction.

From a study of the sketches of these cases and other tests, it is concluded that when $F$ is about 0.68 , the flow in the junction can become critical and hydraulic jump formation is possible. Whether a jump forms or not depends on the discharge ratios of the flow in the main channel extension. The region in the plot of $F$ against $F$ where the hydraulic jump formation is possible is shown in Figure lla. The changing 
state from undular jumps to weak jumps then back to undular jumps again is shown in Figure 19, in which the ratio of the amplitude to depth of the jumps are plotted aga inst the discharge ratio, $Q_{m} / Q$.

\subsection{Surface Discontinuity}

A deep cut or an abrupt step on the water surface around the upstream corner of the junction appears in every test except when the flow in the channels is very slow or deep, that is, $F<0.1$.

When $F$ is about 0.25 , this discontinuity is initiated from the upstream corner of the junction. This surface discontinuity has a nearly constant length of about half the channel width but its width varies with different flow conditions. Depth measurements show that the lowest point of this surface discontinuity can be less than $1 / 6$ of the mean depth of flow in the junction. It points diagonally across the junction (Figure 20 and Plate 6). As the flow becomes faster in the main channel, this surface discontinuity rotates anticlockwise. When $F$ is about 0.7 , it points directly at the downstream corner across the entrance to the branch channel (Figure 21 and Plate 7). When $F$ is near its limit of 0.85 , the deep cut inclines slightly into the branch (Figure 22 and Plate 8 ). Its ultimate position coincides with the boundary of the recirculation region where the flow separates from the upstream corner of the junction.

The effect of the surface discontinuity on the flow condition is not known by the author. A brief description of its existence is intended here to provoke further investigation into its nature in a dividing flow problem particularly. 


\section{EXTENSION OF THE THEORY}

In this chapter an attempt is made to extend the theoretical analys is of a dividing flow for different angle of intersection, $\theta$, and width of the branch channel, $b_{b}$ (Figure 7.1).

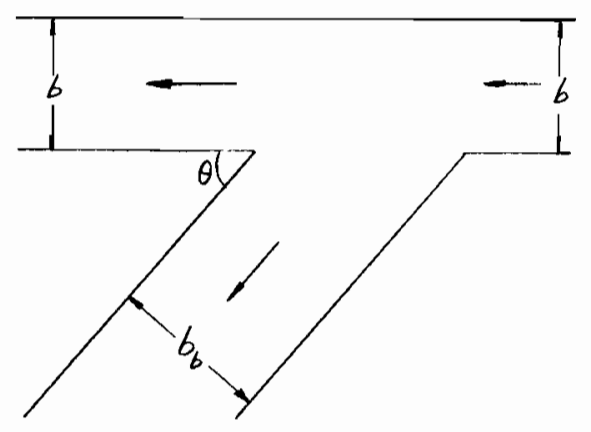

Fig. 7.1 Dividing Flow

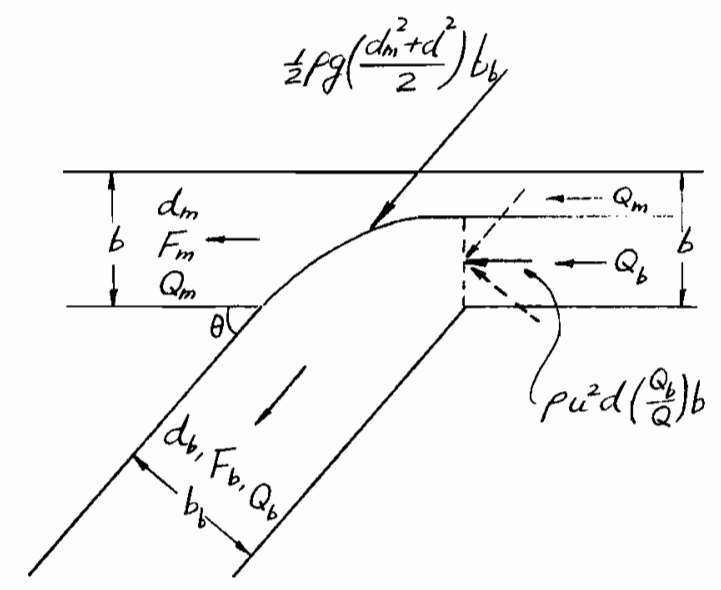

Fig. 7.2 Force diagram-in the direction of the branch channel

It has been shown previously that the determination of the flow condition in the branch requires the application of a contraction coefficient, $c$, to account for the presence of the extensive recirculation region. It is also seen for the case when $\theta=90^{\circ}$, that $c$ depends both on $Q_{b} / Q$ and $F$. In general, no unique relationship of $c$ as a function of $Q_{b} / Q$ and $F$ can be stated, either experimentally or theoretically, without a complete understanding of the development of the recirculation region in different ranges of $Q_{b} / Q$ and $F$. To verify the validity of the theory for any $\theta$ and $b_{b}$ ' the arbitrary choice of $c$ is to be avoided. It is proposed in the following sections to analyze some experimental results by $\operatorname{Grace}^{(3)}$ with the theory developed in this study. In this way, it is hoped that the distribution of $c$ for different values of $Q_{b} / Q$ and $F$ can be generalized. If the values of $c$ for a particular geometry of the model so generalized conform with the physical behaviour of the flow in the branch, then the validity of the theory is verified.

Assuming the energy loss is negligible, the momentum and energy equations in the main channel extension for any angle of intersection $\theta$ are the same as equations 
(4.7) and (4.10) respectively, as in the case when $\theta=90^{\circ}$. However, a variation in $\theta$ will necessarily produce a different distribution of the flow at the junction. For example, when free-overfall conditions exist in both the main channel extension and the branch, and $\theta=90^{\circ}$, it has been found that $Q_{b} / Q$ is about $20 \%$. When $\theta$ is reduced to, say $30^{\circ}$, even under the same free-overfall conditions, it is expected that more flow will be diverted into the branch. For the same Froude number, $F$, in the main channel, once the $Q_{m} / Q$ ratio is determined for a desired angle of intersection, $\theta$, the variation in $F_{m}$ can be determined either experimentally or theoretically. Figures $1 \mathrm{la}$ and $b$ for $\theta=90^{\circ}$ are applicable to any value of $\theta$ since $F_{m}$ is invariant to any change in $\theta$. The only varying parameter is the discharge ratio $Q_{m} / Q$ or $Q_{b} / Q$. Thus, the whole problem of a dividing flow for any angle of intersection is reduced to the determination of the discharge ratio $Q_{b} / Q$ under the given cond itions such as $\theta$ and $F$.

To determine the discharge ratio, $Q_{b} / Q$, take the case when the angle of intersection is $\theta$, and the width of the branch is $b_{b}$. The hydrostatic force developed in the direction of the branch due to the expanding flow at the junction is

$$
\frac{1}{2} \rho g\left(\frac{d^{2}+d_{m}^{2}}{2}\right) b_{b}
$$

The branch is set at an angle to the main channel. Consequently, a component of the momentum of flow in the main channel should be included in the direction of the branch (Figure 7.2). This component of momentum is estimated as $\rho u^{2} d\left(\frac{Q_{b}}{Q}\right) b \cos \theta$. The momentum equation for the branch can be written as

$$
\frac{1}{2} \rho g\left(\frac{d_{m}^{2}+d^{2}}{2}\right) b_{b}+\rho u^{2} d\left(\frac{Q_{b}}{Q}\right) b \cos \theta=\frac{1}{2} \rho g d_{b}{ }^{2} b_{b}+\rho u_{b}^{\prime}{ }^{2} d_{b} c b_{b} 7.1
$$

where $b_{b}$ is the width of the branch channel, $\theta$ is the angle of intersection and the other symbols are the same as those used in equation (4.17).

When only the influence of $\theta$ on the theory of a dividing flow is required, the width of all sections of the whole model is taken to be the same, that is $b_{b} / b=1$. In a later section, a general discussion on the influence of the width ratio will be given. 
The assumption made is $d_{m} / d=1$ (section 4.2.1). Equation (7.1) can be arranged to give

$$
\left(\frac{d_{b}}{d}\right)\left[1+2 F^{2}\left(\frac{Q_{b}}{Q}\right) \cos \theta\right]-\left(\frac{d_{b}}{d}\right)^{3}=2 F^{2}\left(\frac{Q b}{Q}\right)^{2} / c
$$

where $F$ is the Froude number of the flow in the main channel.

Another useful relationsh ip between $F, d_{b} / d$, and $Q_{b} / Q$ can be derived from the continuity equations. From

$$
\begin{aligned}
& Q=u d b \\
& Q_{b}=u_{b} d_{b} b
\end{aligned}
$$

it can be shown that

$$
F^{2}=\frac{\left(d_{b} / d\right)^{3} F_{b}^{2}}{\left(Q_{b} / Q\right)^{2}}
$$

When equations (7.2) and (7.3) are evaluated simultaneously, it can be seen that $c$ is determined for a particular combination of $Q_{b} / Q$ and $d_{b} / d$ once the value of $F_{b}$ is fixed. In the following analysis the values of $c$ will be found for cases under free-overfall condition for various values of $\theta$. The values of $F_{b}$ under freeoverfall condition in the branch are found to attain a limiting value which changes only slightly over a large range of $Q_{b} / Q$ ratios near unity. For $\theta=90^{\circ}$, the Froude number of the branch flow for the free-overfall condition in this model is found experimentally equal to 0.68 for $Q_{b} / Q$ higher than 0.6 (Figure $12 a$ ). For values of $Q_{b} / Q$ between 0.6 and 0.2 , the limiting values of $F_{b}$ are slightly lower than 0.68 but the exact values of $F_{b}$ are not clearly indicated. For $Q_{b} / Q<0.2$, the free-overfall condition in the branch can no longer exist as the gate is raised to reduce the discharge. Thus, we are justified in assuming that the limiting value of $F_{b}$ under free-overfall condition is constant irrespective of the variation in $Q_{b} / Q$ or $F$. 
For $\theta=90^{\circ}$, the limiting value of $F_{b}$ is 0.68 . As $\theta$ approaches zero, the limiting value of $F_{b}$ approaches the limit of $F$ of the main channel and is less than 0.85 due to asymmetry of flows. So it can be expected that the $F_{b}$ limit will increase corresponding to a decrease in $\theta$. In the following analys is, four cases will be examined. The values of $\theta$ chosen are $30^{\circ}, 60^{\circ}, 90^{\circ}$ and $120^{\circ}$ and the values of $F_{b}$ limit under free-overfall condition are chosen to be $0.76,0.73,0.68$ and 0.65 for each $\theta$ respectively.

The procedure adopted in the analys is is as follows. Take the case when $\theta=60^{\circ}$. The experimental results by Grace ${ }^{(3)}$ of $Q_{b} / Q$ and $d_{b} / d$ for $\theta=60^{\circ}$ and $b_{b} / b=1$ are used. Since it is found that the points in his plot with the lowest $d_{b} / d$ ratios correspond to the free-overfall condition, a limiting curve connecting these points is drawn. The values of $Q_{b} / Q$ and $d_{b} / d$ are read from the graph and the assigned value of $F_{b}$ for these points is 0.73 . Thus from equation (7.3), the corresponding value of $F$ for different points on this line can be calculated. Since for a particular point on this line, the values of $Q_{b} / Q, d_{b} / d$ and $F$ are known, the value of $c$ can be calculated from equation (7.2). In this way, the variation of $c$ with respect to the variation in $F$ and $Q_{b} / Q$ can be found. The results are plotted in Figure 23. Following the same procedure, the values of $\mathrm{c}$ and $\mathrm{F}$ for $\theta$ equal to $30^{\circ}, 90^{\circ}$, and $120^{\circ}$ are plotted in the same figure. In general, $c$ is a function of $F, F_{b}, Q_{b} / Q$ and $\theta$.

It will be observed in Figure 23 that the value of $c$ remains practically constant and equals one for a large range of $Q_{b} / Q$ ratios and $F$. It has to be noted that $c$ can attain a maximum value of one physically. From equation (7.2) any calculated. value of $c$ greater than one is automatically taken to be as one.

The fact that different methods of depth measurement in the branch channel adopted by Grace and the author may have great significance in the interpretation of the results deserves special attention. It is expected that under freeoverfall condition in the branch, a hydraulic jump will form across the branch channel when the conditions are favourable such as the incoming Froude number greater than 0.7 (Figure 7.3). When the angle of intersection $\theta$ decreases, the flow in the branch will become faster and the hydraulic jump will move downstream. 


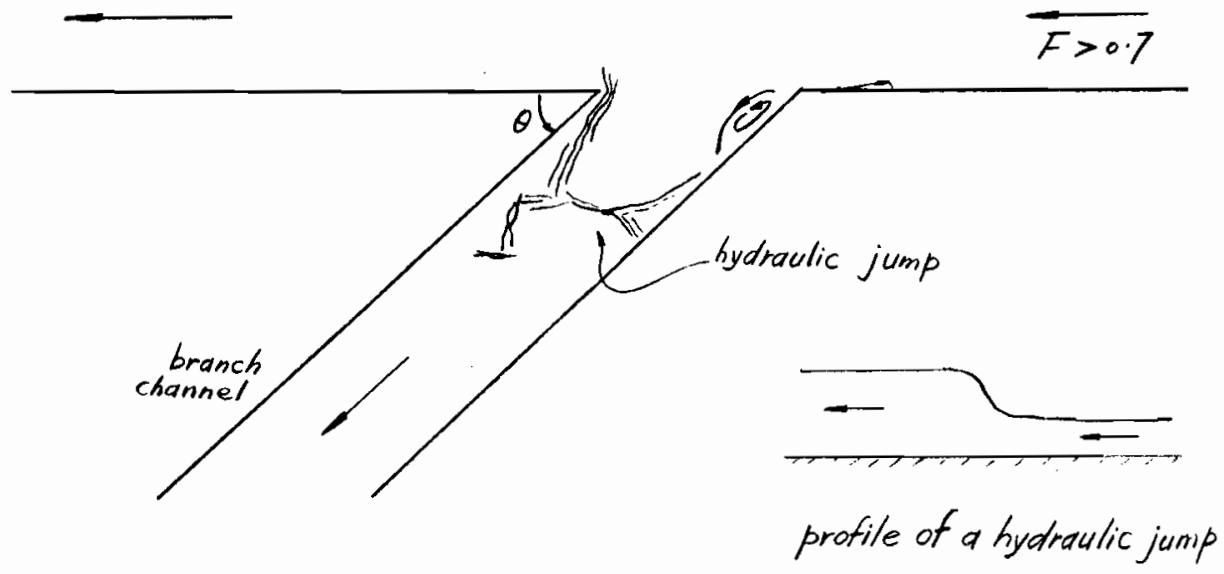

Figure 7.3 Hydraulic jump in a branch channel.

Depth measurement taken upstream or downstream of the hydraulic jump will yield a corresponding small or large ratio of $d_{b} / d$ for a particular $Q_{b} / Q$ ratio. The depth measurement of the branch flow in Grace 's experiment was taken at "2.25 feet from the intersection of channel centerline "(3) . There is no indication in Grace's report, however, to reveal whether the depth measurements have all been taken downstream (or upstream) of the jump or partly upstream and partly downstream. A lower $d_{b} / d$ value will be recorded in Grace's experiment when the depth measurement in the branch channel was taken upstream instead of downstream of the jump.

In the experiments performed by the author, the depth measurement in the branch was taken at 34 inches from the centre of the junction. In every test, it has been checked that the depth measurement along the centreline was either a constant or a minimum. Consequently, this station for depth measurement was assured to be free from the effect of the jump and the back water effect from the exit end of the channel

It is suspected that some of the depth measurements recorded by Grace were taken upstream of the jump in the branch. This fact is specially convincing when $\theta$ is small since the jump will move further downstream in the branch. A depth measurement upstream of the jump yeilds a smaller value of $d_{b}$. A small value of $d_{b} / d$ will produce a corresponding change in $F$, as shown in equation (7.3) and a decrease in the value of $c$ as obtained from equation (7.2). This statement applied to all values of $\theta$, and it is particularly significant in the case when $\theta$ is small. The early drop of the $F$ 
and $c$ curves shown for $\theta=30^{\circ}$ in Figure 23 can be explained as follows. If the depth measurement had been taken at a station which is downstream of the jump, and is free from the back water effect from the exit end of the branch, a greater $d_{b} / d$ will be obtained. Thus a higher value of $F$ (note that the ratio $\left(d_{b} / d\right)^{2} /\left(Q_{b} / Q\right)^{3}$ in controlling the variation of $F$ in equation (7.3)) and $c$ are obtained for all $Q_{b} / Q$ ratios. An increase in $F$ and $c$ values will shift the $F$ and c curves for $\theta=30^{\circ}$ more closely to follow the general trend of the corresponding $F$ and $c$ curves for other values of $\theta$.

With reference to Figure 23, the following observations can be stated for a dividing flow under free-overfall condition in the branch when the angle of intersection of the branch with the main channel extension is $\theta$.

1. When all the inflow is being diverted into the branch at the junction, that is $Q_{b} / Q=1$, there is a definite Froude number, $F$, which the flow in the main channel can obtain irrespective of the total inflow, but depends only on the angle $\theta$. In the case when $\theta=90^{\circ}$, it is approximately equal to 0.5 . A decrease in $\theta$ corresponds to an increase in $\mathrm{F}$ or a faster state of flow in the main channel. Th is is in agreement with the physical nature of the problem. When the angle of intersection $\theta$ is fixed, a continuous decrease in $Q_{b} / Q$ ratio corresponds to a faster state of flow in the main channel. Consequently, a continuous rise in $F$ results until the limiting value of $F$, about 0.85 , is attained (sections 4.5 and 5.1 ).

This limit of $F=0.85$ is achieved experimentally when $Q_{b} / Q$ is about 0.25 corresponding to free-overfall conditions in both the main channel extension and the branch. Further decrease in $Q_{b} / Q$ below 0.25 can only be achieved by raising the gate in the branch while the value of $F$ remains constant at its limiting value of 0.85 as the flow in the junction becomes supercritical.

2. In the above discussion, it is stated that the limiting value of $F=0.85$ is achieved when $Q_{b} / Q$ is reduced to about 0.25 . It has been mentioned in Section 6.2 for the case when $\theta=90^{\circ}$ that $F=0.85$ is attained when $Q_{b} / Q$ is equal to 0.21 . This inconsistency is owing to the different methods in measuring $d_{b}$ as stated earlier. The dependence on $\theta$ is expected to be less significant. 
3.

From Figure 23, it is seen that for a large range of $Q_{b} / Q$, from 0.6 to 1 , the value of $c=1$ holds irrespective of the state of flow in the main channel. For the case when $\theta=90^{\circ}$, it has been observed experimentally that there is no reverse flow necessary to be accounted for when $Q_{b} / Q>0.5$ (Figure 18). Further checking of the experimental results by Grace as plotted in Figure 23, verifies that this is the case even when the angle of intersection varies. Deviation from the above statement in the cases of $\theta=60^{\circ}$ and $30^{\circ}$ is due mainly to the erroneous measurement of $d_{b}$ as stated earlier.

4. In the range of $Q_{b} / Q$ between 0.3 and 0.6 , a variation in the choice of c for different values of $F$ and $Q_{b} / Q$ is clearly indicated in Figure 23. From this plot, it is suggested that when $Q_{b} / Q$ is decreasing, a decrease in the value of $c$ is appropriate. A decrease in $c$ indicates that the recirculation region in the branch grows in size. This fact bears out the physical phenomenon when the discharge in the branch is decreased (Figures 16 and 17).

5. In the range of $Q_{b} / Q$ less than 0.3 , the value of $F$ will be constant at its limiting value of about 0.85 while the variation in the discharge ratio $Q_{b} / Q$ corresponds only to a variation in the value of $F_{b}$. In practice, this region of small discharge in the branch is of little interest. Further, it is expected that in this range of $Q_{b} / Q$, the variation of $c$ will not have a simple relationship with the variation in $Q_{b} / Q$. It is observed experimentally that the recirculation region in the branch grows quickly in both length and width for a small change in $Q_{b} / Q$.

6. For $\theta=90^{\circ}$, the values of $\mathrm{c}$ as tabulated in Table III are plotted against $Q_{b} / Q$ and $F$ in Figure 23. It has to be noted that the choice of $c$ in this case is a step-wise increment to cope with the experimental observations. This choice of $c$ by the author is used not only to describe the flow in the free-overfall condition corresponding to a $F_{b}$ limit of 0.7 . It is applied to other values of $F_{b}$ (smaller than $0.7)$ as well. 
The study of a dividing flow problem can be resolved in two different cases. One relates to the flow in the main channel extension and the other relates to the flow in the branch. The former case is relatively simple and does not depend on the choice of $\theta$. The branch flow is complicated.

To describe a branch flow, the parameters $c, F_{b^{\prime}} F$ and $Q_{b} / Q$ are important. By fixing any one of these four parameters, say $F_{b}=$ constant, it is possible to produce correlation curves between $F, Q_{b} / Q$ and $c$ similar to those shown in Figure 23 for the free-overfall condition in the branch. Under the free-overfall condition and for any $\theta, F_{b}$ is assumed to have its limiting value constant for all discharge ratios $Q_{b} / Q>0.3$. However, model tests show that $F_{b}$ limit is constant only for $Q_{b} / Q>0.6$. For $0.3<Q_{b} / Q<0.6$, th is $F_{b}$ limit varies slightly (Figure 12a). For other constant values of $F_{b}$ or constant gate elevations (they are not the same entity), other sets of similar curves can be generated.

When another coordinate of $F_{b}$ is added perpendicular to those shown in the plane of Figure 23 , the limits being from $F_{b}$ limit to zero, it can be visualized that these $F$ and $c$ curves actually extend into a three-dimensional space where $F_{b}$ forms the third dimension. It is important to understand that there is an interrelationsh ip between $c, F$ and $Q_{b} / Q$, while $F_{b}$ is assumed to be a constant.

To summarize, it can be stated that when $Q_{b} / Q>0.6$, there is no extensive recirculation region in the branch longer than four times the width of the channel. The choice of $c$ in this range of flow is one for all values of $F$. This choice of $c$ is applicable to all values of $\theta$ as well. When $0.25<Q_{b} / Q<0.6$, the recirculation region in the branch grows to such a size that its contraction effect on the channel width becomes important. In th is range of flow, the value of $F_{b}$ decreases corresponding to a decrease in the discharge ratio $Q_{b} / Q$. The cornesponding increase in $F$ is relatively slow. Rapid flowsarealso observed in the junction. When the discharge ratio is less than 0.25 , the growth of the recirculation region in the branch is fast. It is difficult to correlate accurately its growth rate with the decrease rate of the discharge ratio. When $Q_{b} / Q<0.25$, rapid flows are found across the entrance to the branch. The Froude number in the main channel remains constant at its limiting value of 0.85 . Variations in the discharge ratio $Q_{b} / Q$ below 0.25 change only the flow condition in the branch. 
Alternatively, it can be stated that the determination of the discharge ratio, $Q_{b} / Q$, depends mainly on the extent of the recirculation region in the branch. It has been shown that a contraction coefficient is a function of $F, F_{b}, Q_{b} / Q$ and $\theta$. There is further complication involved in the interrelationship among the different parameters. At the present time, an analytical approach to deal with a dividing flow problem is difficult. To gain a better understanding of the problem, one should resort to model tests.

In this final section of the chapter, the effect of different branch width on a dividing flow is discussed briefly. If the width ratio is $b_{b} / b=n$, equations (7.2) and (7.3) are modif ied respectively to

$$
\left(\frac{d_{b}}{d}\right)\left[1+2 F^{2}\left(\frac{Q_{b}}{Q}\right) \frac{\cos \theta}{n}\right]-\left(\frac{d_{b}}{d}\right)^{3}=2 F^{2}\left(\frac{Q_{b}}{Q}\right)^{2} / c
$$

$$
\text { and } F^{2}=\frac{\left(\frac{d_{b}}{d}\right)^{3}\left(F_{b}\right)^{2} n^{2}}{\left(\frac{Q_{b}}{Q}\right)^{2}}
$$

Depending on whether $n>1$ or $n<1$, the effect of the width ratio, $n$, is to relocate the $F$-curves in the plot of $d_{b} / d$ against $Q_{b} / Q$ accordingly.

However, it is evident from Figure 23 that the contraction coefficient $c$ and the limit of $F_{b}$ are the decisive factors in determining the discharge ratio $Q_{b} / Q$. Without a thorough understanding of the development of the recirculation region in the branch, this simple theory is insufficient to solve the problem of adividing flow in an open channel. 


\section{CONCLUSION}

For a dividing flow at low Froude numbers in a $\theta=90^{\circ}$ model, the momentum and energy principles are equally applicable to describe the flow in the main channel extension. This is true provided that a proper choice of a hydrostatic force developed at the junction due to the expanding flow is included in the momentum equation. At high inflow Froude numbers, it is found that the energy loss due to the formation of hydraulic jumps cannot be neglected. The crude model used in this analys is is not accurate enough to produce sat isfactory results. The flow in the branch can also be predicted from the momentum principle when an appropriate contraction coeff icient is applied to account for the presence of extensive recirculation regions in the branch.

To verify the validity of the theory for a general angle of intersection $\theta$, the experimental results by Grace ${ }^{(3)}$ are correlated with the theory to determine the contraction coefficient. It is intended that if the contraction coefficients so determined conform with the expected physical nature of the flow condition, the validity of the theory is justified. It is found that the theory predicts the flow sat isfactorily when $Q_{b} / Q>0.6$ which corresponds tothe cases when the recirculation region in the branch does not extend far downstream and its presence is insignificant. However, when $Q_{b} / Q<0.6$, the theory is ineffective. In this range of flow, it is difficult to determine the value of the contraction coefficient.

It is found that the contraction coefficient, $c$, is a function of $F, F_{b}$ ' $Q_{b} / Q$ and $\theta$. Basically, these parameters are interrelated. A graphical representation of the values of $c$ to correlate with other parameters is shown in Figure 23. The variable chosen is $Q_{b} / Q$, while the variations of $c$ and $F$ are correlated with each other and with $Q_{b} / Q$. On the same $p l o t$, the parameter is $\theta$ and the values of $F_{b}$ will form the third dimension. No simple representation of $c$ can be established. For each individual problem, a model test is recommended.

Analytically, a dividing flow in an open channel can only be solved effectively when the problem concerning the development of the recirculation region is better understood. Other interesting features observed during this study are the 
hydraulic jumps and the surface discontinuity.

In practice, a hydraulic jump is undesirable in a channel because of its elevated surface above the mean depth of flow and of its damage to the sides of the channel. A hydraulic jump makes its appearance in the main channel extension when the incoming flow from the main channel is fast with a Froude number $F$ greater than 0.7 . Its effect on the flow has not been determined precisely in this experiment and further study into its influence on a dividing flow is certainly warranted.

The nature of the surface discontinuity provides another challenging problem to the study of a dividing flow. It appears at the upstream corner of the junction whenever the incoming flow in the main channel has a Froude number $F>0.25$. Its appearance has been observed to be associated with the wavelets in most cases. Its nature has not been determined in this work. No mention of its occurrence has been found by the author in currently available literature.

One important quantitative conclusion can be drawn in this study. Under free-overfall conditions in this simple model, it is found that about $21 \%$ of the total discharge will be diverted into the branch channel at the junction. A discharge more (or less) than this quantity can be diverted into the branch channel only by raising the sliding gate in the main channel extension (or the branch channel). The influence of the sliding gates on the dividing flow is clearly indicated in Figures 14 and 15. In Figure 18, other observable results of a dividing flow showing when an extensive reverse flow or hydraulic jumps can be expected in this $\theta=90^{\circ}$ model are summarized. 
1. Chow, V.T.

2. Bower, C.E.

3. Grace Jr., J.L.

4. Kandaswamy, P.K., Rouse, $\mathrm{H}$.

5. Krishnappa, G., Seetharamiah, K.

6. Patrabiramiah, Rajaratnam, N.

7. Rajaratnam, N.
"Open Channel Hydraulics, "McGrawHill Book Co., Inc., New York, 1959.

"Study of Open Channel Junctions", Part $\checkmark$ of "Hydraulic Model Studies for Whitting Field Naval Air Station, Milton, Florida, St. Anthony Hydraulic Laboratory", Jan. 1950.

"Division of Flow in Open Channel Junctions", Master Thesis, Alabama Polytechnic Institute, June, 1958.

"Characteristics of Flow Over Terminal Weir and Sill ", Hydraulic Division, ASCE Proc. Paper 1345, August 1957.

"A New Method of Predicting the Flow in a $90^{\circ}$ Branch Channel, "La Houille Blanche, No. 7, November 1963

"A New Method in Predicting Flow in a Branch Channel ", Irrigation and Power Journal, New Delhi, January 1960.

"Constant Velocity Concept in Supercritical Branch Flow, "Irrigation and Power Journal, Vol. 19, No. 1, New Delhi, January 1962.

8. Rouse, $\mathrm{H}$.
"Discharge Characteristics of the Free Overfall", Civil Engineering, Vol. 6, No. 4, 1936. 
9. Taylor, E.H.

10. Thiruvengadam, A.

11. Yoshimi, Y., Stelson, T.E.
"Flow Characteristics at Rectangular Open Channel Junctions", Transactions ASCE Vol. 109, Paper 2223, 1944.

"Flow in a Branch Channel ", Water Power, Vol. 14, 1962.

"Grit Unbalance in Sewage Flow Division", ASCE Proc. Paper 3493, April, 1963. 
TABLE I

EXPERIMENTAL RESULTS

Note: $\quad \frac{Q_{m}}{Q}=1-\frac{Q_{b}}{Q}$

\begin{tabular}{|c|c|c|c|c|c|c|c|c|c|c|c|}
\hline \multicolumn{2}{|c|}{$\begin{array}{l}\text { Test Q } \\
\text { No. cfs }\end{array}$} & $\begin{array}{l}Q_{m} \\
c f s\end{array}$ & $\begin{array}{l}Q_{b} \\
c f s\end{array}$ & $\begin{array}{l}d \\
\mathrm{ft} .\end{array}$ & $\begin{array}{l}d_{m} \\
\mathrm{ft} .\end{array}$ & $\begin{array}{l}d_{b} \\
f t .\end{array}$ & $\frac{Q_{b}}{Q} \%$ & $\frac{d b}{d}$ & $F$ & $\mathrm{~F}_{\mathrm{m}}$ & $b$ \\
\hline & 0.085 & 0.051 & 0.034 & 0.271 & 0.274 & 0.268 & 40.5 & 0.990 & & 0.093 & 0.066 \\
\hline & 0.08 & 0 & 0.084 & 0.160 & 0.166 & & 100.0 & & & & \\
\hline & 0.0 & 0.011 & .073 & & 5 & 24 & 87. & & & & \\
\hline & & & 0 & & 24 & & 0 & & & & \\
\hline & & & 0.035 & & 0.1 & 0.187 & & & & & \\
\hline & 0.08 & & .071 & & & 0.127 & 84. & & & & \\
\hline & & & .025 & & & & 30 & & & & \\
\hline & 0 & & 0 & & 6 & 8 & 0 & & & & \\
\hline & 0 & & 0.028 & & 2 & & 25. & & & & \\
\hline & 0 & & 0 & & & & 0 & & & & \\
\hline & & & 0 & & & & & & & & \\
\hline & 0 & & 0.006 & & 5 & 3 & & 97 & & & \\
\hline & & & 044 & & 5 & & 41. & & & & \\
\hline & 0.1 & & 079 & & & & & & & & \\
\hline & & & & & & & & & & & \\
\hline & & & 084 & & 5 & 44 & 77 & & & & \\
\hline & & & 046 & & 7 & 2 & 42. & & & & \\
\hline & & & & & & & & & & & \\
\hline & & & & & & & & & & & \\
\hline & & & & & & & & & & & \\
\hline & & & & & 7 & & & & & & \\
\hline 2 & & & & & & & & & & & \\
\hline & & & & & & & & & & & \\
\hline & & ( & & & & & & & & & \\
\hline & & & & & 339 & 328 & & & & & 0.136 \\
\hline & & & 057 & & 382 & .378 & & & & & 0.064 \\
\hline & & & .015 & & 0.332 & & & & & & 0.021 \\
\hline 28 & & & & & 0.298 & & & & & & 0.091 \\
\hline & & 037 & & & & & & & & 0.075 & \\
\hline & & 0 & & & & & & & & & \\
\hline & & & & & & & & & & & 0.474 \\
\hline 2 & & & & & 0.215 & .200 & 38.2 & 0.975 & & 0.221 & 0.153 \\
\hline & 0.134 & $0.1<4$ & 0.010 & 0.241 & 0.244 & $0.24 \cdot 1$ & 1.8 & & 0.301 & 0.274 & 0.022 \\
\hline
\end{tabular}




\begin{tabular}{|c|c|c|c|c|c|c|c|c|c|c|c|}
\hline $\begin{array}{l}\text { Test } \\
\text { No. }\end{array}$ & $\begin{array}{l}Q \\
\text { cfs }\end{array}$ & $\begin{array}{l}Q_{m} \\
c f s\end{array}$ & $\begin{array}{l}Q_{b} \\
c f s\end{array}$ & $\begin{array}{l}d \\
\mathrm{ft} .\end{array}$ & $\begin{array}{l}d_{m} \\
\mathrm{ft} .\end{array}$ & $\begin{array}{l}d_{b} \\
f t .\end{array}$ & $\frac{Q_{b}}{Q} \%$ & $\frac{d_{b}}{d}$ & $F$ & $F_{m}$ & $F_{b}$ \\
\hline 4 & 0.136 & 0.136 & 0 & 0.253 & 0.254 & 0.254 & 0 & 1.002 & 0.280 & 0.280 & 0 \\
\hline U & 0.136 & 0.136 & 0 & 0.159 & 0.153 & 0.154 & 0 & 0.968 & 0.568 & 0.596 & 0 \\
\hline 36 & 0.136 & & 0.031 & 0.124 & 0.155 & 0.081 & 22.5 & 0.653 & 0.823 & 0.455 & 0.350 \\
\hline 37 & 0.162 & 0.1 & 0.033 & 0.139 & 0.152 & 0.087 & 20.3 & 0.626 & 0.822 & 0.571 & 0.340 \\
\hline 38 & 0.162 & 0.138 & 0.024 & 0.145 & 0.155 & 0.127 & 14.8 & 0.876 & 0.772 & 0.595 & 0.120 \\
\hline 39 & 0.162 & 0.153 & 0.009 & 0.164 & 0.163 & 0.158 & 5.2 & 0.964 & 0.646 & 0.612 & 0.042 \\
\hline 40 & 0.162 & 0.162 & 0 & 0.175 & 0.168 & 0.173 & 0 & 0.990 & 0.587 & 0.622 & 0 \\
\hline 41 & & & 0 & 0.274 & 0.274 & 0.275 & 0 & 1.002 & 0.299 & 0.299 & 0 \\
\hline 42 & 0.1 & 0.1 & 0.020 & 0.257 & 0.261 & 0.255 & 12.3 & 0.992 & 0.331 & 0.282 & 0.041 \\
\hline 43 & 0.163 & 0.101 & 0.062 & 0.222 & 0.263 & 0.214 & 38.2 & 0.964 & 0.414 & 0.198 & 0.166 \\
\hline 44 & 0.162 & 0.059 & 0.103 & 0.179 & 0.196 & 0.153 & 63.7 & 0.805 & 0.565 & 0.179 & 0.455 \\
\hline 45 & 0.162 & 0 & 0.162 & 0.238 & 0.238 & 0.188 & 100.0 & 0.790 & 0.370 & 0 & 0.523 \\
\hline 46 & 0.084 & 0.061 & 0.023 & 0.152 & 0.158 & 0.150 & 27.4 & 0.987 & .376 & 0.258 & 0.106 \\
\hline 47 & 0.084 & 0.052 & 0.032 & 0.144 & 0.151 & 0.141 & 37.9 & 0.980 & 0.408 & 0.236 & 0.160 \\
\hline 48 & & & 0.040 & & & & & 0.955 & 0.454 & & 0.234 \\
\hline 49 & 0.086 & 0.037 & 0.049 & 0.124 & 0.133 & 0.113 & 56.5 & 0.911 & 517 & 0.202 & 0.336 \\
\hline 50 & 0.084 & 0.029 & 0.055 & 0.115 & 0.126 & 0.092 & 65.5 & 0.800 & 0.573 & 0.171 & 0.526 \\
\hline 51 & 0.084 & 0.043 & 0.041 & 0.1 & 0.115 & 0.075 & 49.4 & 0.734 & 384 & 0.294 & 0.532 \\
\hline 52 & 0.084 & 0.049 & 0.035 & 0.120 & 0.122 & 0.097 & 41.8 & 0.809 & 0.537 & 0.304 & 0.309 \\
\hline 53 & 0.086 & 0.057 & 0.029 & 0.119 & 0.129 & 0.113 & 33.2 & 0.950 & 550 & 0.325 & 0.197 \\
\hline 54 & 0.086 & 0.066 & 0.020 & 0.130 & 0.136 & 0.125 & 23.5 & 0.962 & 481 & 0.344 & 0.120 \\
\hline 55 & 0.086 & 0.074 & 0.012 & 0.1 & 0.143 & 0.1 & 13.4 & 0.985 & & 360 & 0.060 \\
\hline 56 & 0.086 & 0.084 & 0.002 & 0.126 & 0.124 & 0.124 & 2.5 & 0.985 & .505 & 0.504 & 0.013 \\
\hline 57 & 0.086 & 0.077 & 0.009 & 0.117 & 0.120 & 0.113 & 10.7 & 0.966 & 564 & 0.484 & 0.064 \\
\hline 58 & 0.086 & 0.069 & 0.017 & 0.107 & 0.115 & 0.097 & 20.0 & 0.906 & 645 & 0.462 & 0.150 \\
\hline 59 & 0.087 & & & 0.099 & 0.1 & & 26.9 & & & & 59 \\
\hline 60 & 0.087 & 0.072 & 0.015 & 0.092 & 0.096 & 0.073 & 16.7 & 0.793 & 322 & 0.643 & 0.194 \\
\hline 61 & 0.087 & 0.068 & 0.019 & 0.092 & 0.098 & 0.053 & 21.6 & 0.576 & .828 & 0.590 & 0.409 \\
\hline 62 & 0.087 & 0.077 & 0.010 & 0.102 & 0.107 & 0.093 & 11.4 & 0.912 & .706 & 0.640 & 0.092 \\
\hline 63 & 0.103 & 0.096 & 0.007 & 0.1 & 0.118 & 0.1 & 7.1 & 0.950 & 670 & 0.625 & 0.052 \\
\hline 64 & 0.105 & 0.090 & 0.015 & 0.1 & 0.116 & 0.09 & 14.2 & 0.891 & 763 & 0.605 & 0.129 \\
\hline 65 & 0.105 & 0.08 & 0.019 & 0.108 & 0.114 & 0.079 & 17.6 & 0.732 & .781 & 0.594 & 0.219 \\
\hline 66 & 0.107 & 0.084 & 0.023 & 0.108 & 0.112 & 0.059 & 21.5 & 0.546 & 0.800 & 0.605 & 0.425 \\
\hline 67 & 0.105 & 0.071 & 0.034 & 0.109 & 0.122 & 0.067 & 32.8 & 0.615 & 0.771 & 0.438 & 0.524 \\
\hline 68 & 0.105 & 0.077 & 0.028 & 0.110 & 0.121 & 0.085 & 26.9 & 0.773 & 0.759 & 0.481 & 0.300 \\
\hline 69 & 0.105 & 0.090 & 0.015 & 0.127 & 0.130 & 0.118 & 14.3 & 0.930 & .613 & 0.507 & 0.098 \\
\hline 70 & & 0.099 & 0.007 & 0.135 & 0.135 & 0.131 & 6.9 & 0.970 & 0.565 & 0.522 & 0.040 \\
\hline 71 & 0.104 & 0.084 & 0.020 & 0.149 & 0.154 & 0.146 & 18.7 & 0.980 & 0.475 & 0.368 & 0.092 \\
\hline 72 & 0.105 & 0.076 & 0.029 & 0.141 & 0.149 & 0.136 & 27.1 & 0.965 & 0.524 & 0.351 & 0.150 \\
\hline 73 & 0.105 & 0.068 & 0.037 & 0.130 & 0.142 & 0.123 & 35.3 & 0.946 & 0.594 & 0.336 & 0.228 \\
\hline 74 & 0.105 & 0.060 & 0.045 & 0.122 & 0.130 & 0.106 & 42.8 & 0.869 & 0.650 & 0.316 & 0.344 \\
\hline 75 & 0.105 & 0.054 & 0.051 & 0.113 & 0.134 & 0.084 & 48.8 & 0.743 & 0.730 & 0.303 & 0.555 \\
\hline 76 & 0.183 & 0 & 0.183 & 0.227 & 0.239 & 0.172 & 100.0 & 0.758 & 0.447 & 0 & 0.672 \\
\hline & 0.162 & 0 & 0.162 & 0.206 & 0.216 & 0.159 & 100.0 & 0.772 & 0.458 & 0 & 0.680 \\
\hline
\end{tabular}




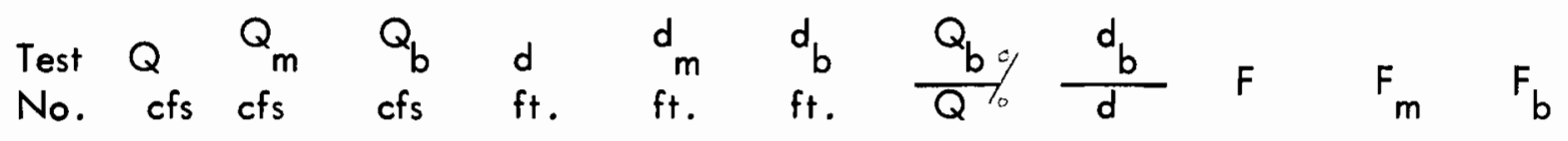

\begin{tabular}{|c|c|c|c|c|c|c|c|c|c|c|c|}
\hline 78 & & 0 & & & & & & 0.779 & 0.434 & 0 & \\
\hline 79 & 0.171 & .015 & 156 & 0.204 & 0.217 & .157 & & 0.770 & 0.490 & & 0.665 \\
\hline 10 & & & & & & & & & & & \\
\hline & & 39 & & & & & & .784 & & 0.112 & \\
\hline & & .052 & & & & & & .733 & 0.555 & 0.162 & 612 \\
\hline & 0. & 0.065 & & & & & & .759 & & & \\
\hline & & 0.082 & 0.088 & & & & & .710 & 709 & 0.289 & \\
\hline & & 0. & & & & & & & & & \\
\hline & & 0.1 & & & & & & 567 & & 0.545 & 470 \\
\hline 7 & & 0 & & & & & & & & & 491 \\
\hline 88 & & & & & & & & & & 032 & \\
\hline & & & & & & & & & & 57 & 39 \\
\hline & 0.1 & 0.0 & & 0.2 & 0.2 & & 82.5 & .772 & 0.541 & 0.084 & .660 \\
\hline 91 & & 0.0 & & & & & & & & 18 & 41 \\
\hline 92 & 0.1 & 0.0 & 0.1 & 34 & 0.2 & & 67.4 & 0.766 & 0.623 & 0.173 & 629 \\
\hline 93 & & 0.0 & & & & & 59.8 & 0.739 & 91 & .230 & \\
\hline 94 & 0. & 0.0 & & & & & & 728 & & 06 & 307 \\
\hline 5 & & 0.1 & & & & & 37.8 & .665 & 0.760 & 0.405 & 532 \\
\hline 6 & & 0.1 & & & & & & & & & \\
\hline 97 & 0.1 & 0.1 & 0.0 & 61 & 0. & & 20.9 & .491 & 0.760 & 0.678 & 0.463 \\
\hline 98 & & & & & & & & & & & \\
\hline 99 & 0.2 & 0.1 & & & & & 7.9 & & & 740 & 354 \\
\hline & & & & & & & 4.7 & 28 & & & 38 \\
\hline 10 & 0.217 & 0.1 & & & 0 . & & 1.0 & 27 & 03 & 753 & 42 \\
\hline . & & 8 & & & 0.1 & & 8.8 & & 0.796 & 0.739 & \\
\hline 10 & 0.2 & 0.2 & & & & & 3.8 & & & 766 & \\
\hline 10 & 0.1 & 0.183 & & 0.176 & 0.162 & & 1.9 & & 0.665 & 0.745 & 13 \\
\hline 10 & 0.1 & 0.176 & & & & & 5.4 & & & 14 & \\
\hline 10 & & 70 & & & & & 8.6 & & 755 & 0.740 & 79 \\
\hline & & 66 & & & & & 11.0 & 745 & & 24. & \\
\hline 108 & & .159 & .027 & .161 & & & 14.5 & 34 & & 10 & 17 \\
\hline & 0.186 & 0.153 & 0.0 & 0.1 & 0. & & 18.1 & 559 & & 06 & 27 \\
\hline & 0.186 & 0.147 & & & & & 20.9 & & & 90 & 96 \\
\hline 11 & 0.202 & 0.16 & 0.041 & 0.1 & 0.1 & & 20.4 & 88 & 88 & 733 & 166 \\
\hline & 0.202 & 0.167 & 0.035 & 0.166 & & & 17.4 & & & 760 & 346 \\
\hline & 0.202 & 0.17 & 0.028 & & & 0. & 13.9 & 16 & 0.782 & 0.776 & 0.222 \\
\hline 11 & 0.202 & 0.180 & 0.022 & & & & 10.8 & & 0.782 & 0.745 & 0.133 \\
\hline & 0.202 & 0.185 & 0.017 & 0.169 & 0.159 & 0.1 & 8.6 & 0.835 & 0.767 & 0.767 & 0.086 \\
\hline & & & & & & & 5.9 & & & 0.762 & 50 \\
\hline & 0.202 & 0.197 & 0.005 & 0.182 & 0.163 & 0.173 & 2.6 & 0.951 & 0.686 & 0.787 & 0.0 .19 \\
\hline & & & 0 & & & & & & 0.665 & 0.784 & 0 \\
\hline & 0.104 & 0.086 & 0.019 & 0.112 & 0.111 & 0.074 & 18.1 & 0.660 & 0.738 & 0.594 & 0.248 \\
\hline & & & & & & & & & & 0.700 & 0.309 \\
\hline re & 0.145 & 0.116 & 0.029 & 0.134 & 0.135 & 0.061 & 20.3 & 0.455 & 0.780 & 0.614 & 0.515 \\
\hline
\end{tabular}


$\begin{array}{llllllllllll}\text { Test } & Q & Q_{m} & Q_{b} & d & d_{m} & d_{b} & Q_{b} & d_{b} & & & \\ \text { No. } & c f s & c f s & c f s & f t . & f t . & f t . & F_{m} & \frac{f_{b}}{d}\end{array}$

$\begin{array}{llllllllllll}122 & 0.145 & 0.126 & 0.019 & 0.135 & 0.126 & 0.098 & 12.8 & 0.727 & 0.772 & 0.747 & 0.159 \\ 123 & 0.145 & 0.130 & 0.015 & 0.135 & 0.133 & 0.117 & 10.2 & 0.867 & 0.772 & 0.709 & 0.097 \\ 124 & 0.145 & 0.137 & 0.008 & 0.145 & 0.137 & 0.132 & 5.5 & 0.910 & 0.694 & 0.713 & 0.044 \\ 125 & 0.145 & 0.145 & 0 & 0.155 & 0.142 & 0.154 & 0 & 0.994 & 0.627 & 0.715 & 0 \\ 126 & 0.165 & 0.159 & 0.006 & 0.159 & 0.147 & 0.152 & 3.7 & 0.955 & 0.685 & 0.742 & 0.027 \\ 127 & 0.165 & 0.152 & 0.013 & 0.150 & 0.146 & 0.136 & 7.5 & 0.906 & 0.746 & 0.720 & 0.065 \\ 128 & 0.166 & 0.149 & 0.017 & 0.149 & 0.142 & 0.119 & 10.5 & 0.798 & 0.761 & 0.731 & 0.112 \\ 129 & 0.166 & 0.144 & 0.022 & 0.148 & 0.138 & 0.098 & 13.5 & 0.662 & 0.771 & 0.739 & 0.192 \\ 130 & 0.166 & 0.136 & 0.030 & 0.148 & 0.137 & 0.083 & 17.9 & 0.561 & 0.771 & 0.710 & 0.329 \\ 131 & 0.166 & 0.130 & 0.036 & 0.148 & 0.134 & 0.069 & 21.4 & 0.466 & 0.768 & 0.701 & 0.518\end{array}$

$\begin{array}{llllllllllll}132 & 0.093 & 0.047 & 0.046 & 0.108 & 0.121 & 0.074 & 49.6 & 0.685 & 0.684 & 0.293 & 0.603 \\ 133 & 0.093 & 0.058 & 0.035 & 0.104 & 0.113 & 0.064 & 37.9 & 0.615 & 0.726 & 0.398 & 0.572 \\ 134 & 0.093 & 0.069 & 0.024 & 0.103 & 0.103 & 0.056 & 25.2 & 0.544 & 0.740 & 0.567 & 0.466 \\ 135 & 0.093 & 0.070 & 0.023 & 0.102 & 0.101 & 0.055 & 24.8 & 0.540 & 0.751 & 0.575 & 0.465 \\ 136 & 0.093 & 0.072 & 0.021 & 0.102 & 0.104 & 0.069 & 22.6 & 0.676 & 0.751 & 0.563 & 0.304 \\ 137 & 0.127 & 0.114 & 0.013 & 0.135 & 0.128 & 0.124 & 9.9 & 0.919 & 0.674 & 0.660 & 0.076 \\ 138 & 0.127 & 0.108 & 0.019 & 0.129 & 0.126 & 0.107 & 14.5 & 0.830 & 0.727 & 0.640 & 0.139 \\ 139 & 0.127 & 0.104 & 0.023 & 0.125 & 0.124 & 0.088 & 17.9 & 0.704 & 0.760 & 0.626 & 0.228 \\ 140 & 0.127 & 0.098 & 0.029 & 0.125 & 0.120 & 0.063 & 23.2 & 0.505 & 0.760 & 0.616 & 0.488 \\ 141 & 0.127 & 0.090 & 0.037 & 0.125 & 0.129 & 0.071 & 29.3 & 0.568 & 0.760 & 0.514 & 0.520 \\ 142 & 0.127 & 0.079 & 0.048 & 0.125 & 0.139 & 0.082 & 37.6 & 0.656 & 0.760 & 0.406 & 0.541 \\ 143 & 0.127 & 0.052 & 0.075 & 0.135 & 0.152 & 0.098 & 58.9 & 0.726 & 0.674 & 0.234 & 0.644 \\ 144 & 0.174 & 0.084 & 0.090 & 0.152 & 0.171 & 0.113 & 52.0 & 0.745 & 0.778 & 0.311 & 0.629 \\ 145 & 0.174 & 0.106 & 0.068 & 0.148 & 0.165 & 0.100 & 38.9 & 0.675 & 0.806 & 0.420 & 0.562 \\ 146 & 0.174 & 0.123 & 0.051 & 0.145 & 0.154 & 0.091 & 29.4 & 0.628 & 0.836 & 0.537 & 0.490 \\ 147 & 0.174 & 0.134 & 0.040 & 0.147 & 0.141 & 0.076 & 22.8 & 0.517 & 0.821 & 0.670 & 0.500 \\ 148 & 0.174 & 0.140 & 0.034 & 0.147 & 0.140 & 0.079 & 19.3 & 0.538 & 0.821 & 0.709 & 0.400 \\ 149 & 0.174 & 0.146 & 0.028 & 0.148 & 0.141 & 0.099 & 16.0 & 0.670 & 0.806 & 0.729 & 0.236 \\ 150 & 0.174 & 0.153 & 0.021 & 0.150 & 0.144 & 0.121 & 12.3 & 0.807 & 0.793 & 0.735 & 0.134 \\ 151 & 0.193 & 0.173 & 0.020 & 0.169 & 0.162 & 0.145 & 10.5 & 0.859 & 0.736 & 0.730 & 0.097 \\ 152 & 0.193 & 0.164 & 0.029 & 0.165 & 0.158 & 0.114 & 15.1 & 0.691 & 0.761 & 0.689 & 0.200 \\ 153 & 0.193 & 0.155 & 0.038 & 0.164 & 0.155 & 0.093 & 19.5 & 0.567 & 0.840 & 0.673 & 0.350 \\ 154 & 0.193 & 0.149 & 0.044 & 0.165 & 0.153 & 0.081 & 22.8 & 0.491 & 0.761 & 0.656 & 0.505 \\ 155 & 0.193 & 0.137 & 0.056 & 0.165 & 0.170 & 0.092 & 29.0 & 0.558 & 0.701 & 0.516 & 0.528 \\ 156 & 0.193 & 0.125 & 0.068 & 0.164 & 0.182 & 0.105 & 35.0 & 0.640 & 0.840 & 0.426 & 0.524 \\ 157 & 0.193 & 0.092 & 0.101 & 0.169 & 0.193 & 0.121 & 52.5 & 0.716 & 0.732 & 0.284 & 0.638\end{array}$


TABLE II

Comparison of momentum and energy principles in determining Froude numbers in the main channel extension

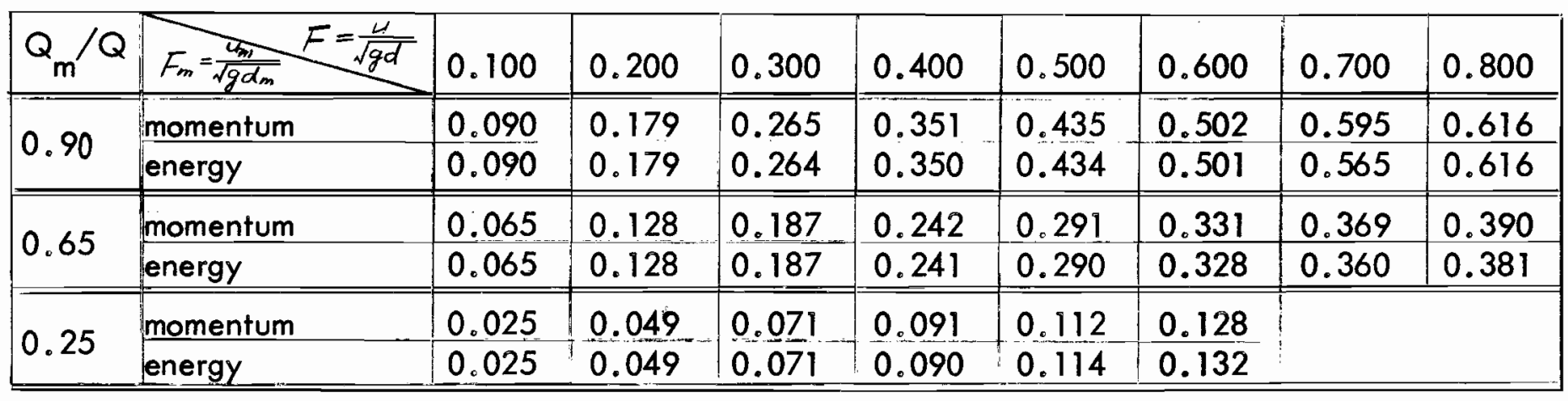

Momentum: Eq. 4.7 $\left(\frac{Q_{m}}{Q}\right)^{2}\left(\frac{F}{F_{m}}\right)^{2}=\left(\frac{\frac{1+\frac{Q_{m}}{Q}}{2}+2\left(\frac{Q_{m}}{Q}\right) F^{2}}{\frac{1+\frac{Q_{m}}{Q}}{2}+2 F_{m}^{2}}\right)^{\frac{3}{2}}$

Energy:

Eq. 4.10 $\left(\frac{Q_{m}}{Q}\right)^{2}\left(\frac{F}{F_{m}}\right)^{2}=\left(\frac{2+F^{2}}{2+F_{m}^{2}}\right)^{3}$

GI 
TABLE III

Choice of contraction coefficients - for flow in branch channel

\begin{tabular}{|c|c|c|c|}
\hline $\begin{array}{c}\text { Range of } \\
\text { F applicable }\end{array}$ & $\begin{array}{c}\text { Effective width } \\
\text { of delivery }\end{array}$ & $\begin{array}{c}\text { Contraction } \\
\text { coefficient } c\end{array}$ & ${\text { Range of } Q_{b} / Q}$ \\
\hline $0.1 \leqslant F \leqslant 0.5$ & 1 & 1 & $0 \leqslant Q_{b} / Q \leqslant 1.0$ \\
\hline $0.5<F \leqslant 0.8$ & $1 / 8$ & 0.125 & $0<Q_{b} / Q \leqslant 0.2$ \\
\hline $0.5<F \leqslant 0.8$ & $1 / 2$ & 0.500 & $0.2<Q_{b} / Q \leqslant 0.4$ \\
\hline $0.5<F \leqslant 0.8$ & 1 & 1 & $0.4<Q_{b} / Q \leqslant 1.0$ \\
\hline
\end{tabular}




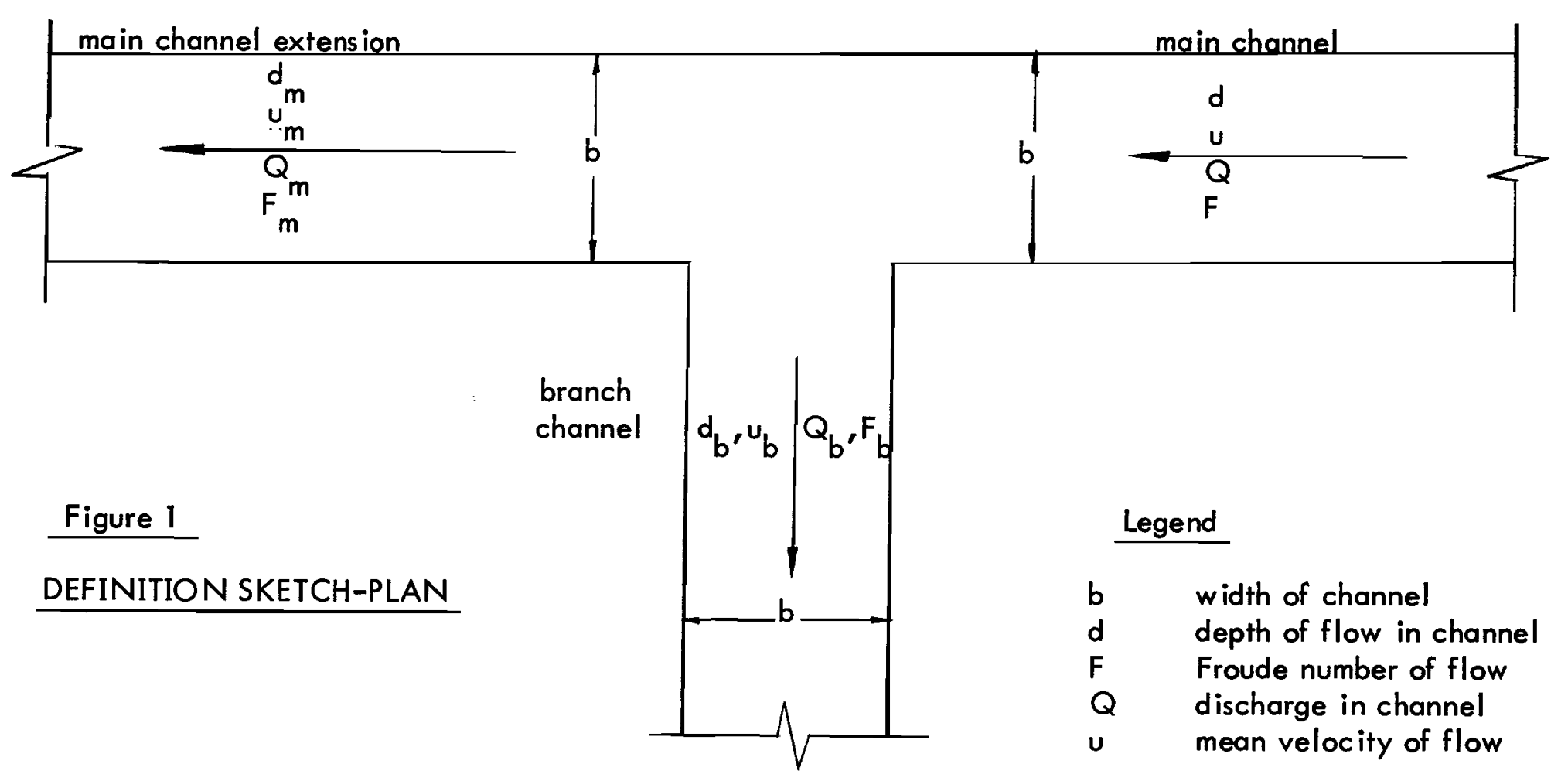




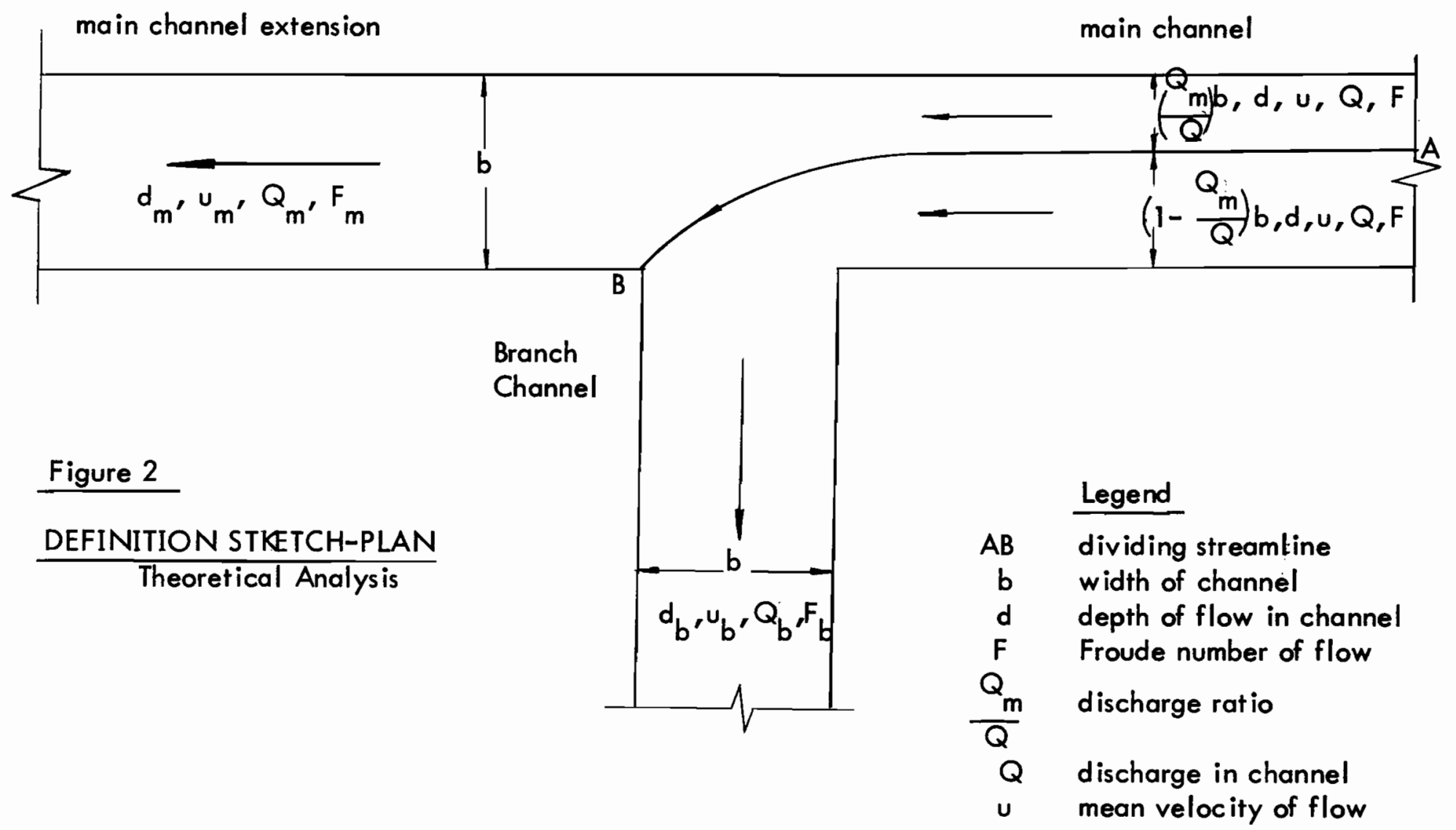




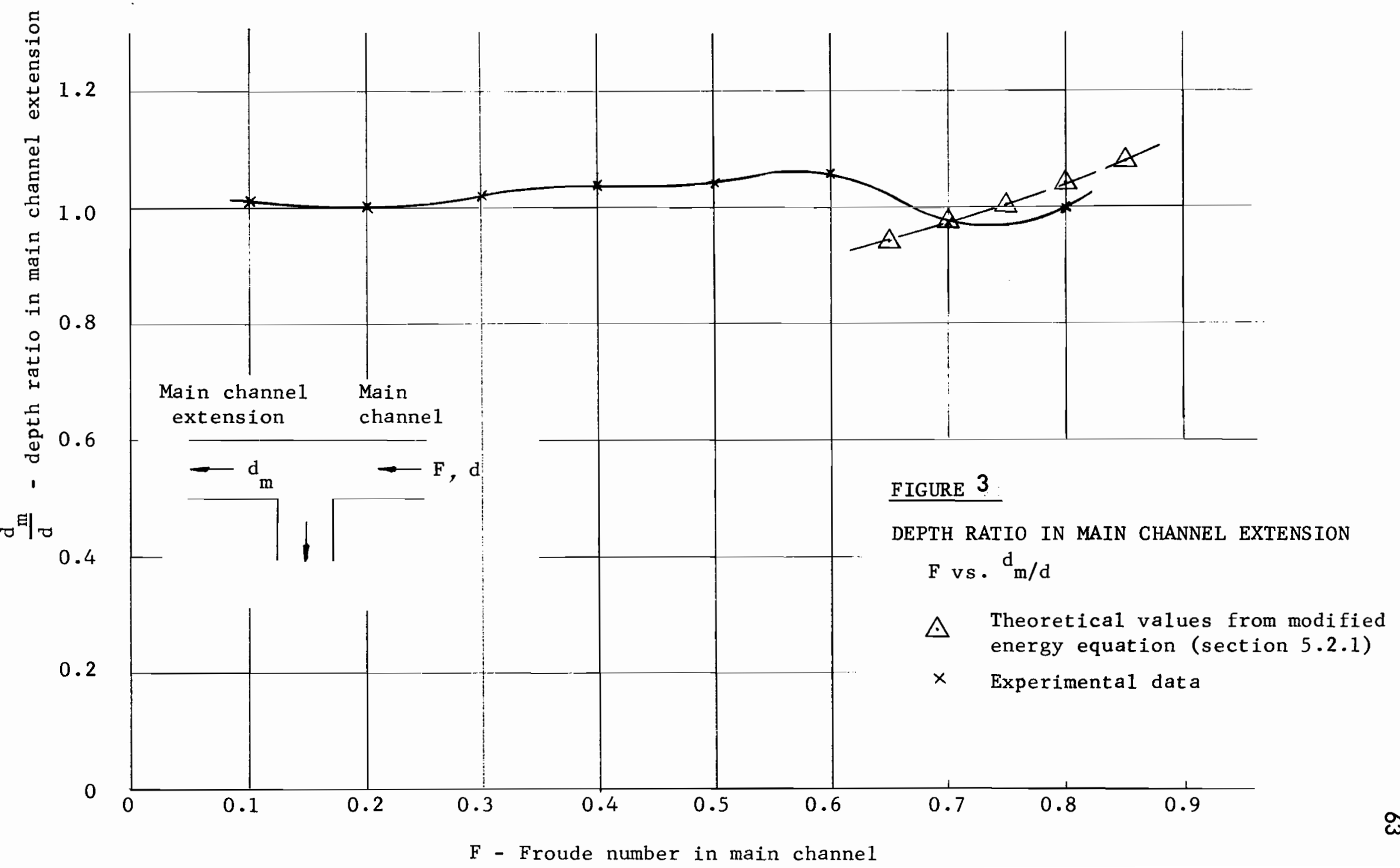




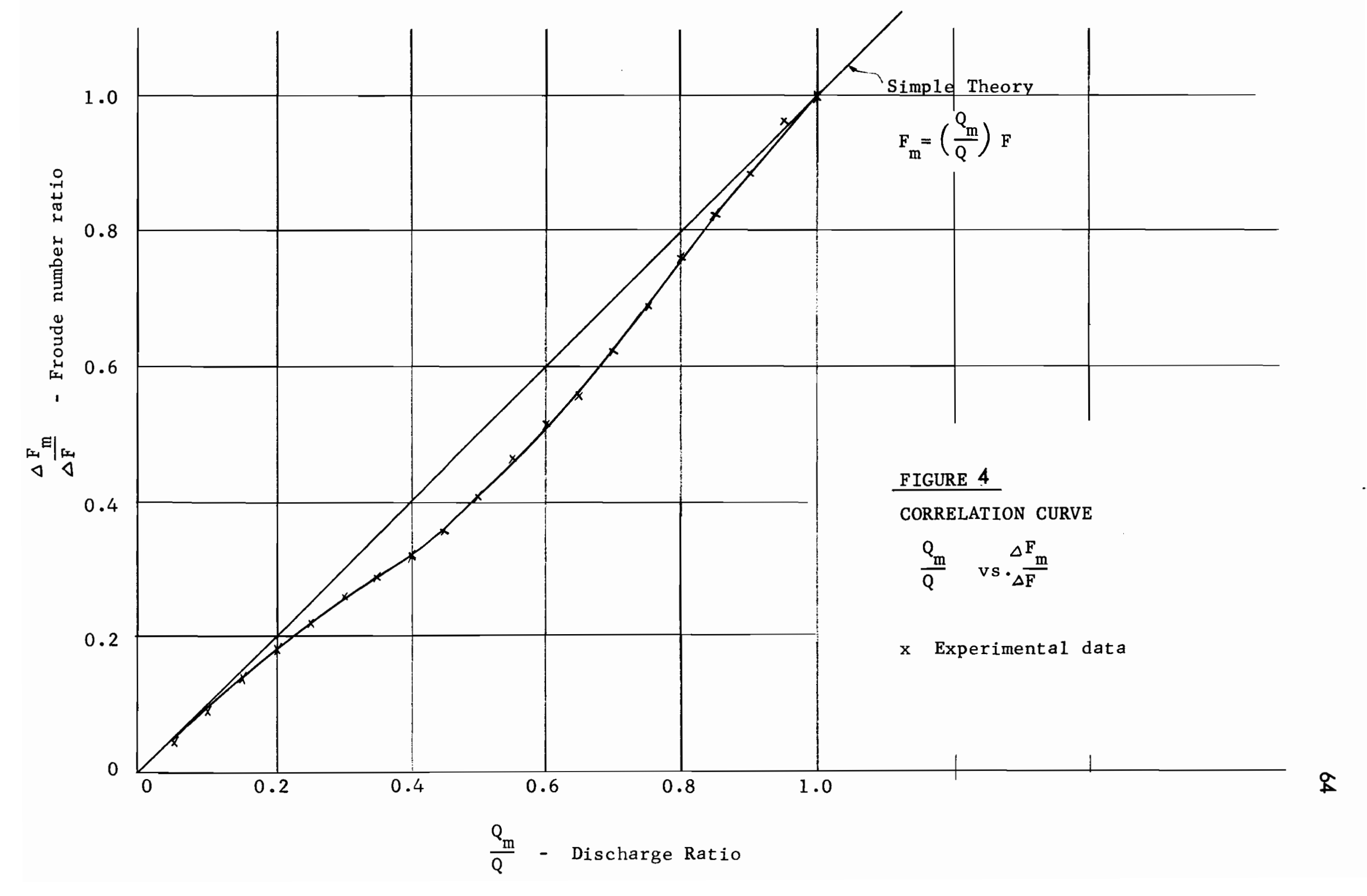




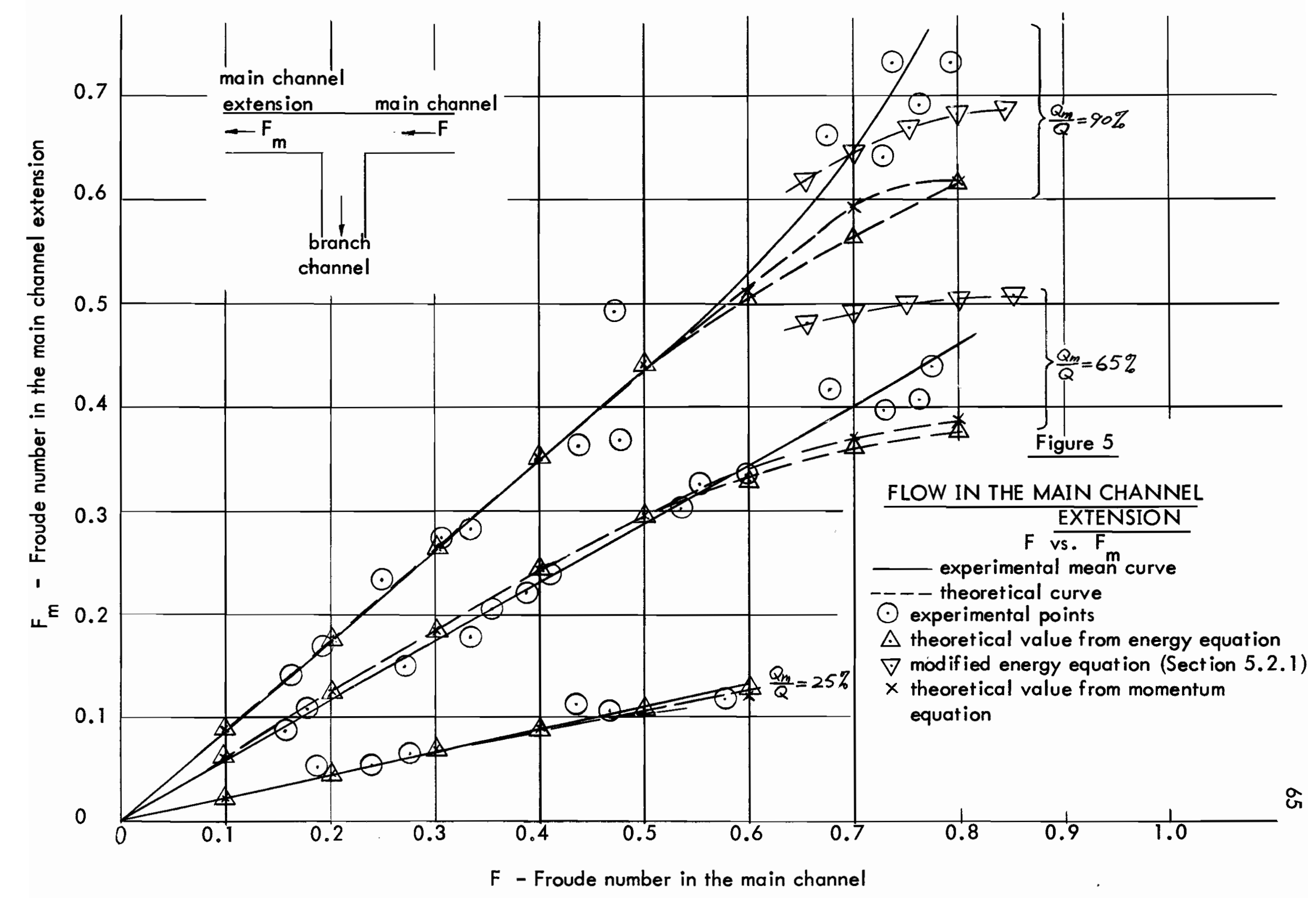




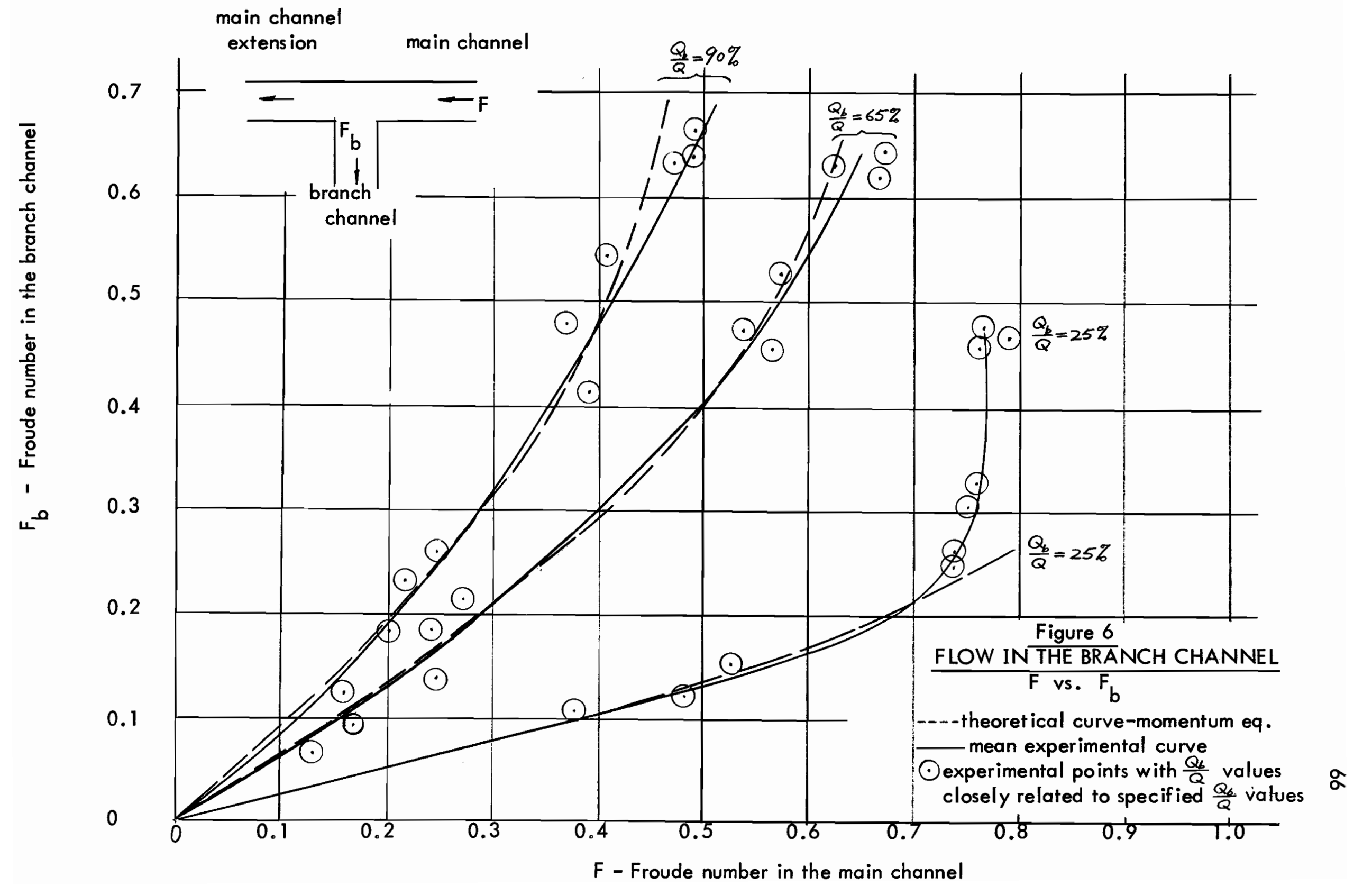




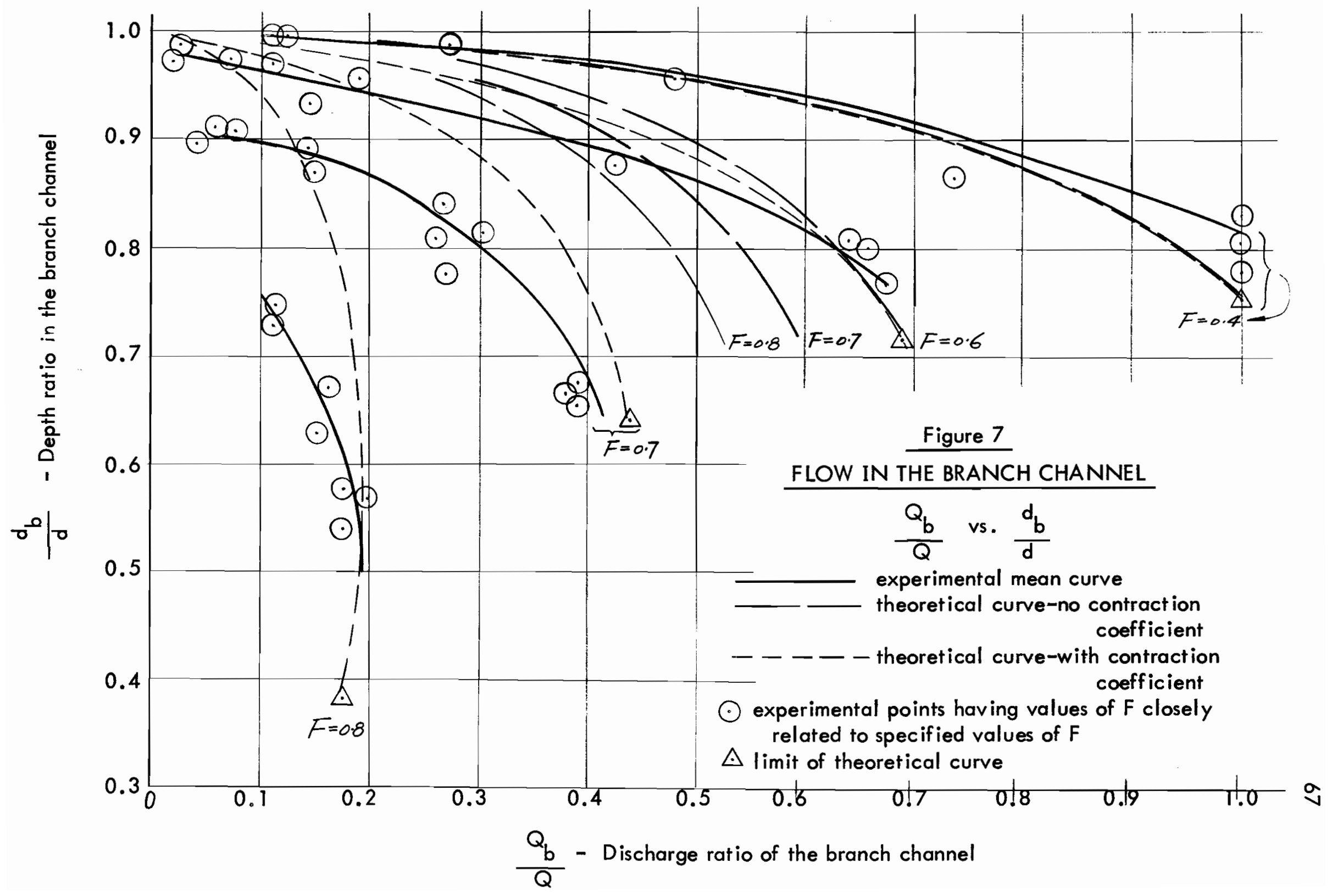




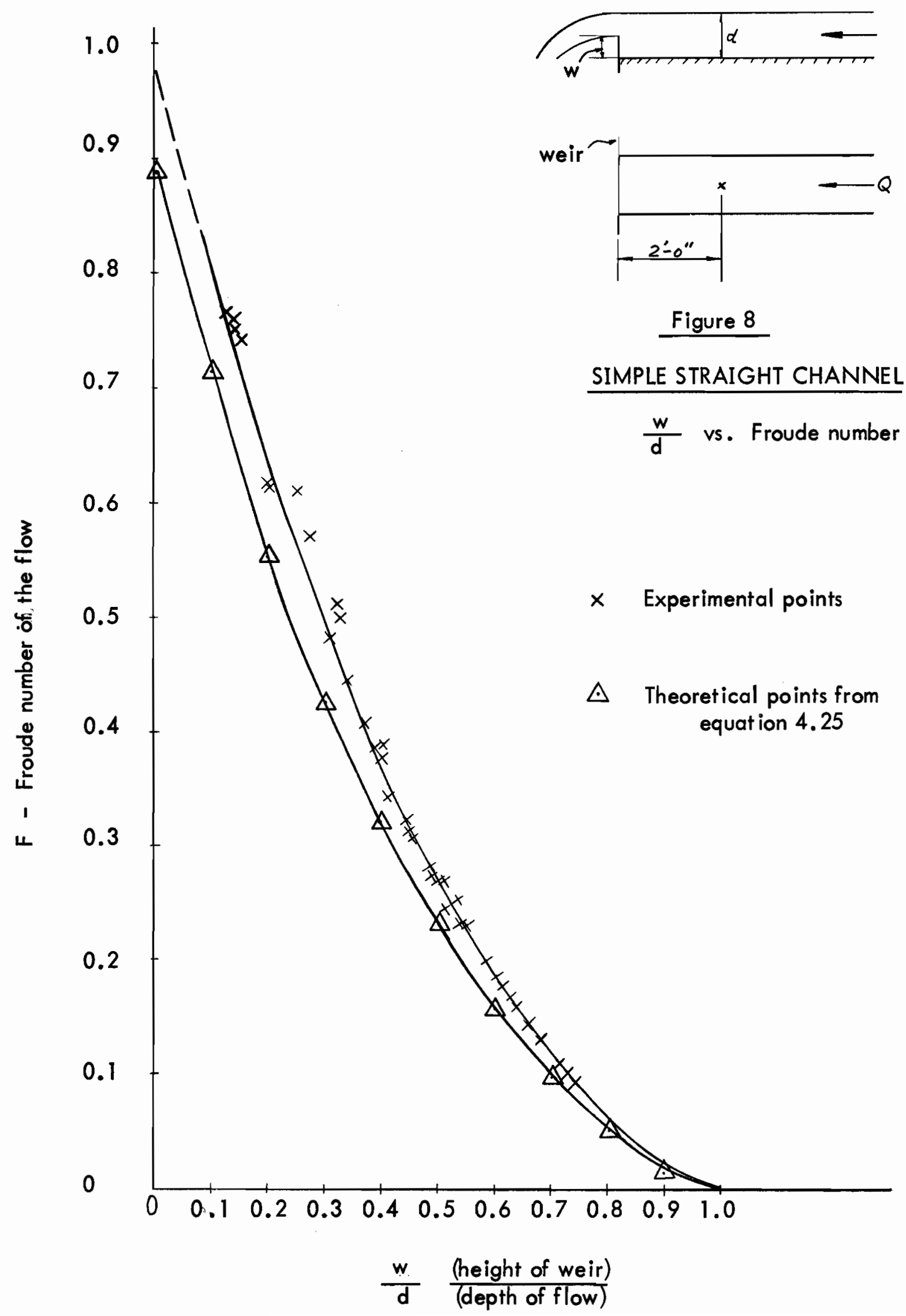




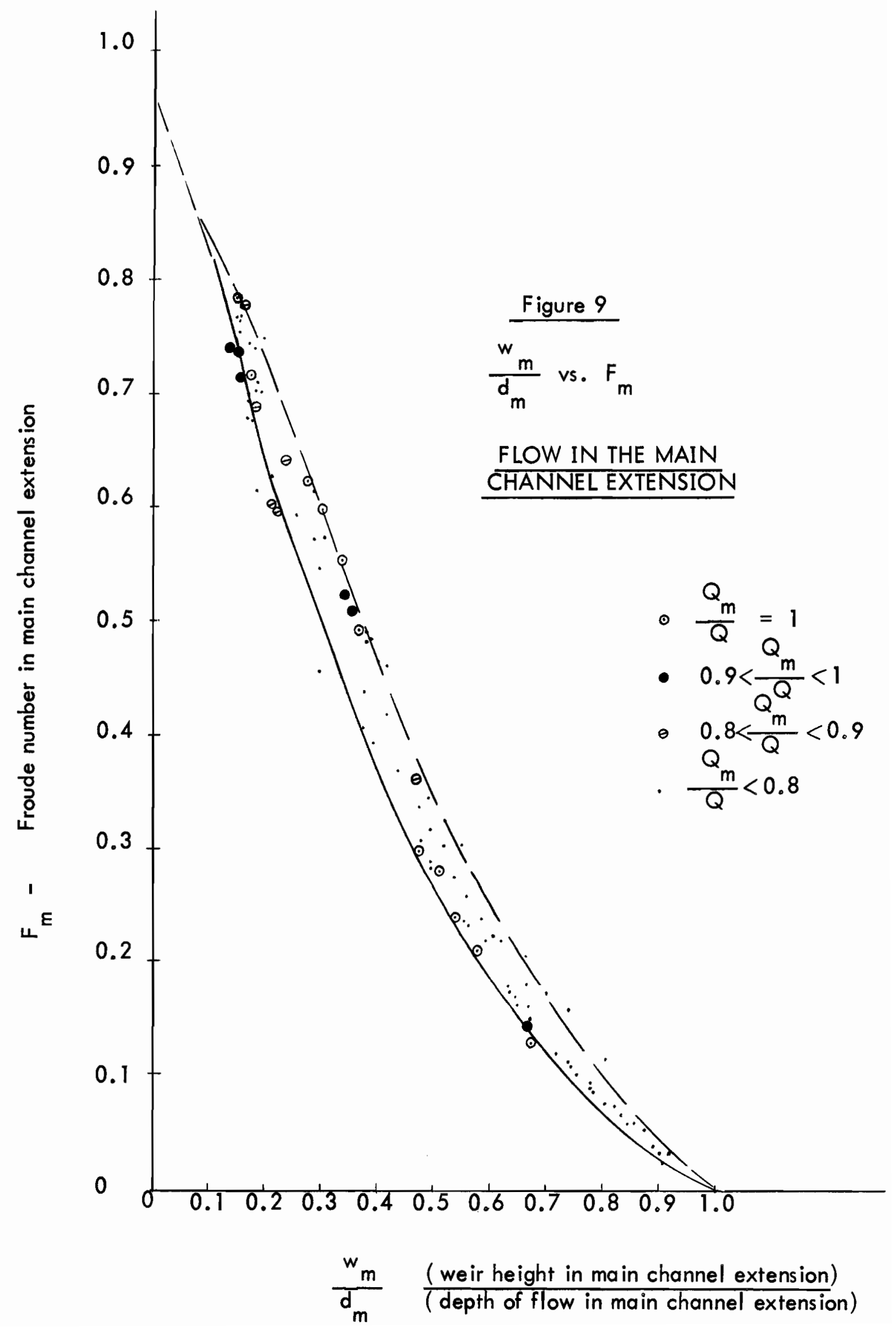




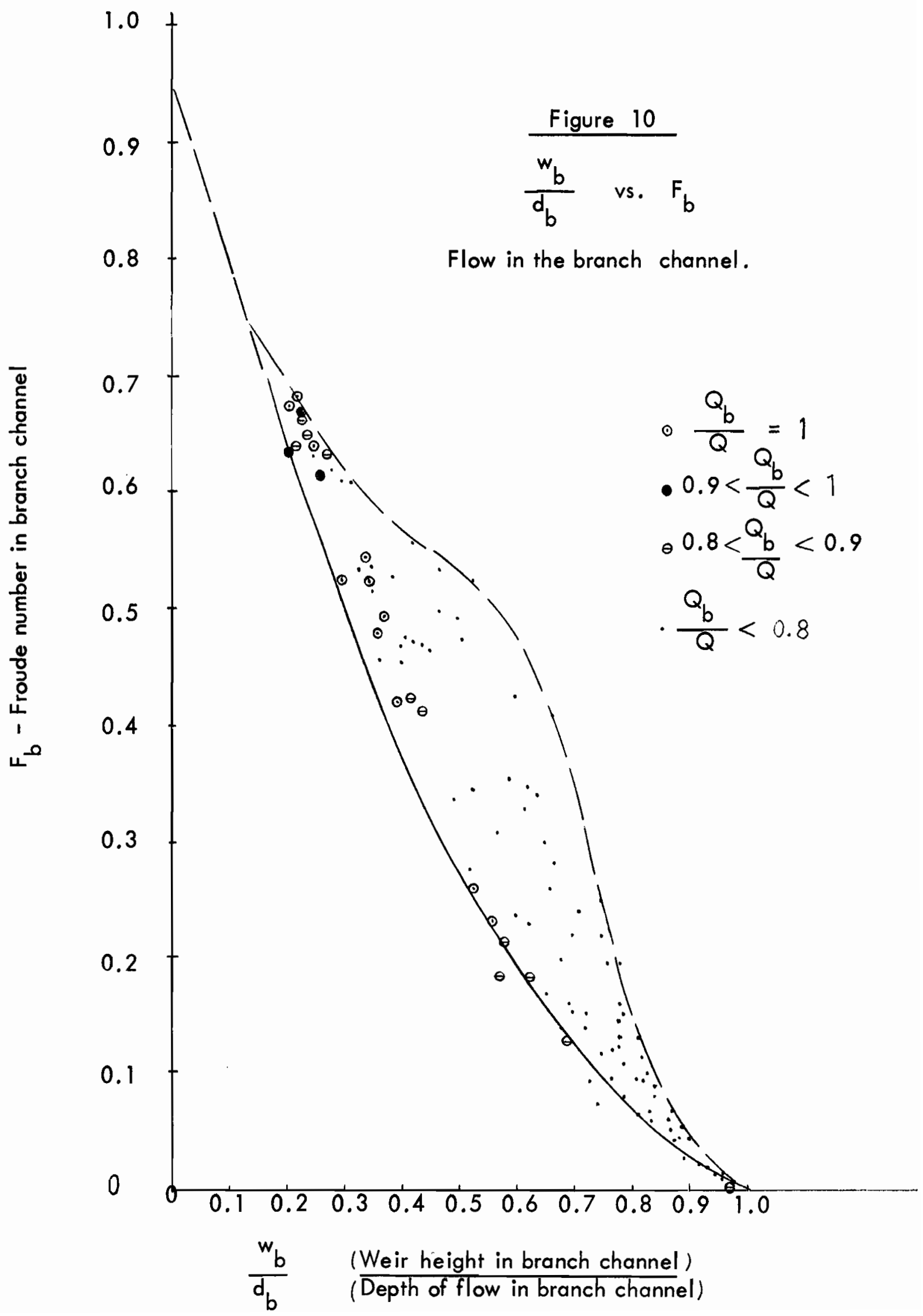



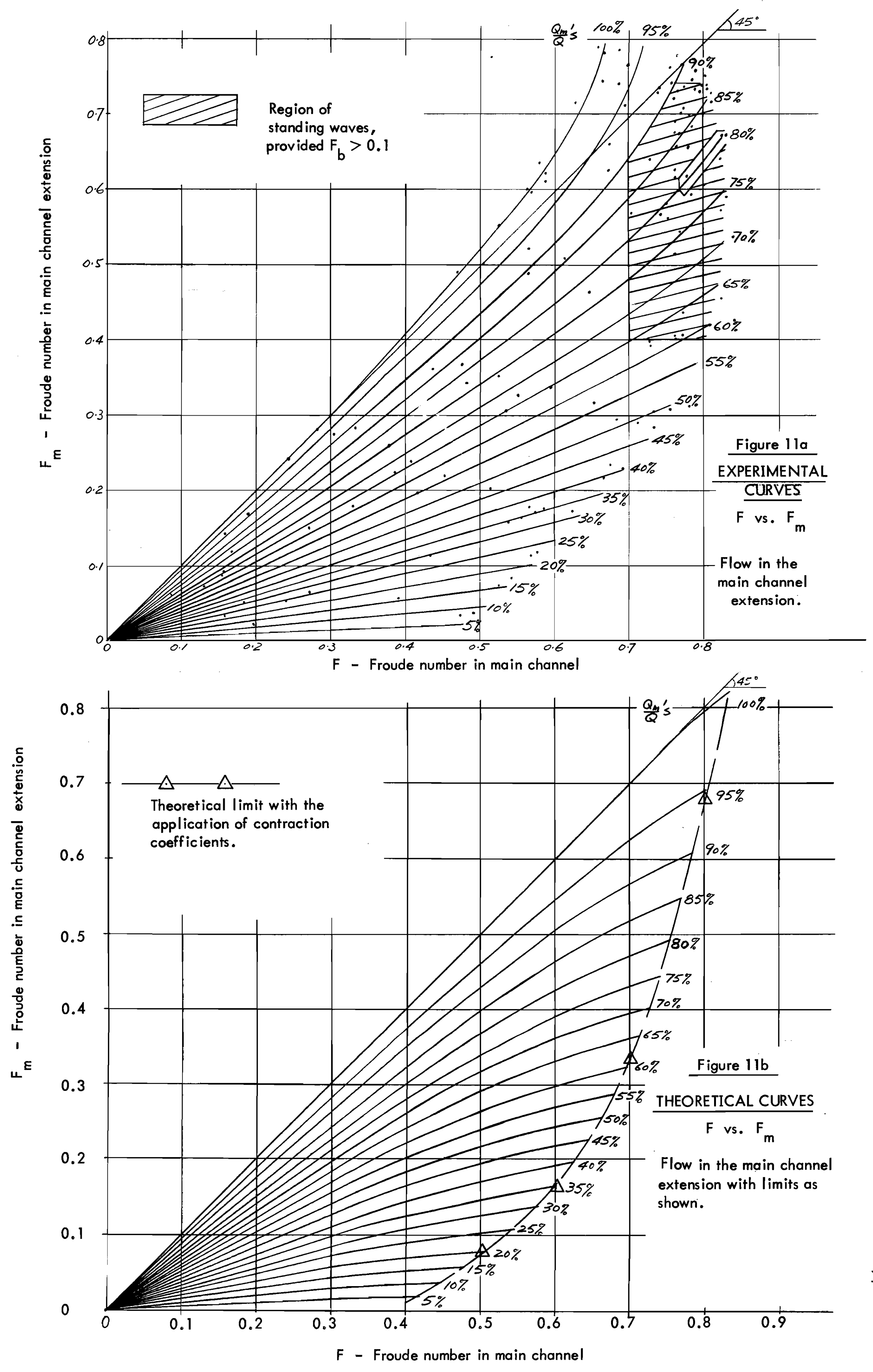

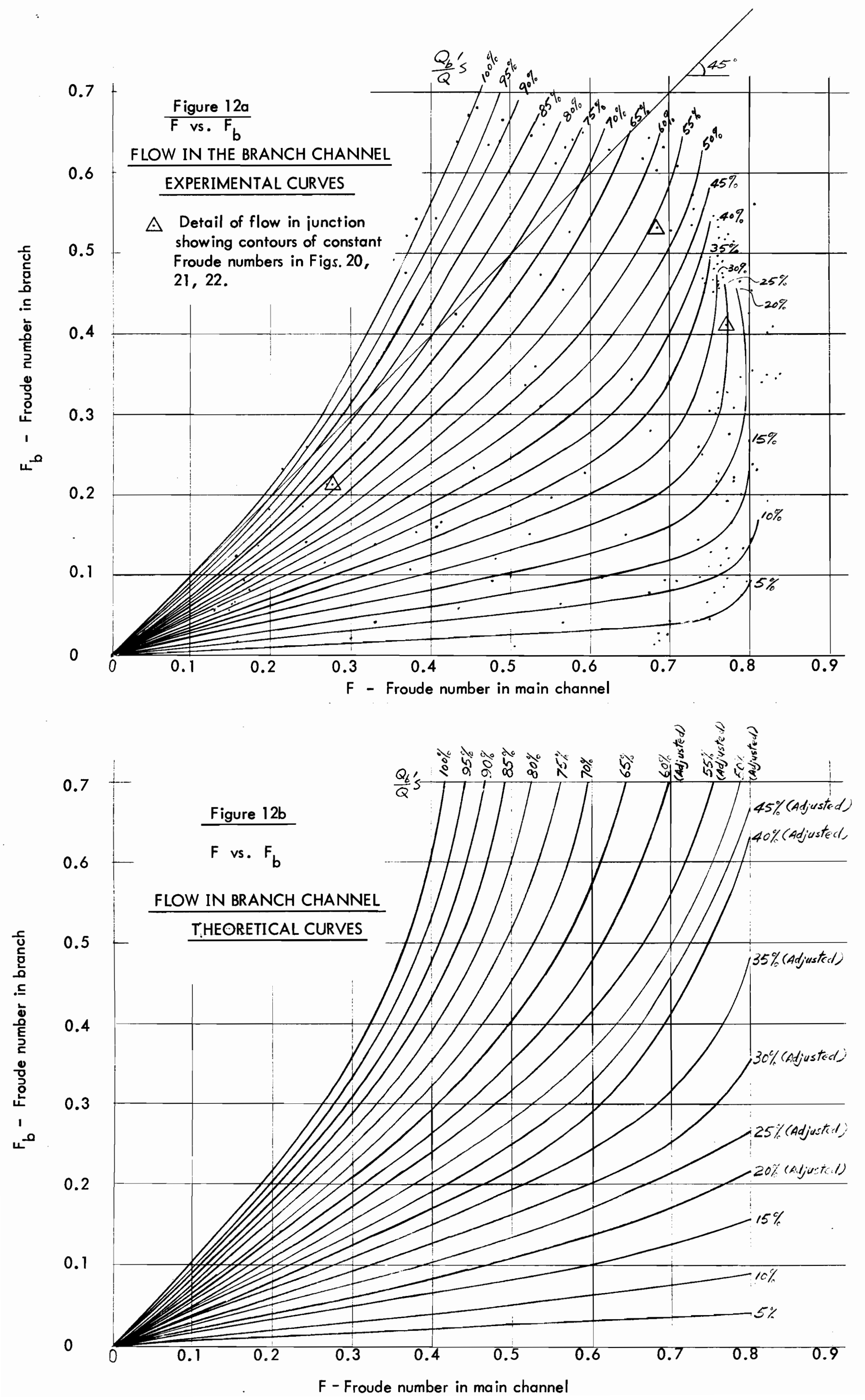

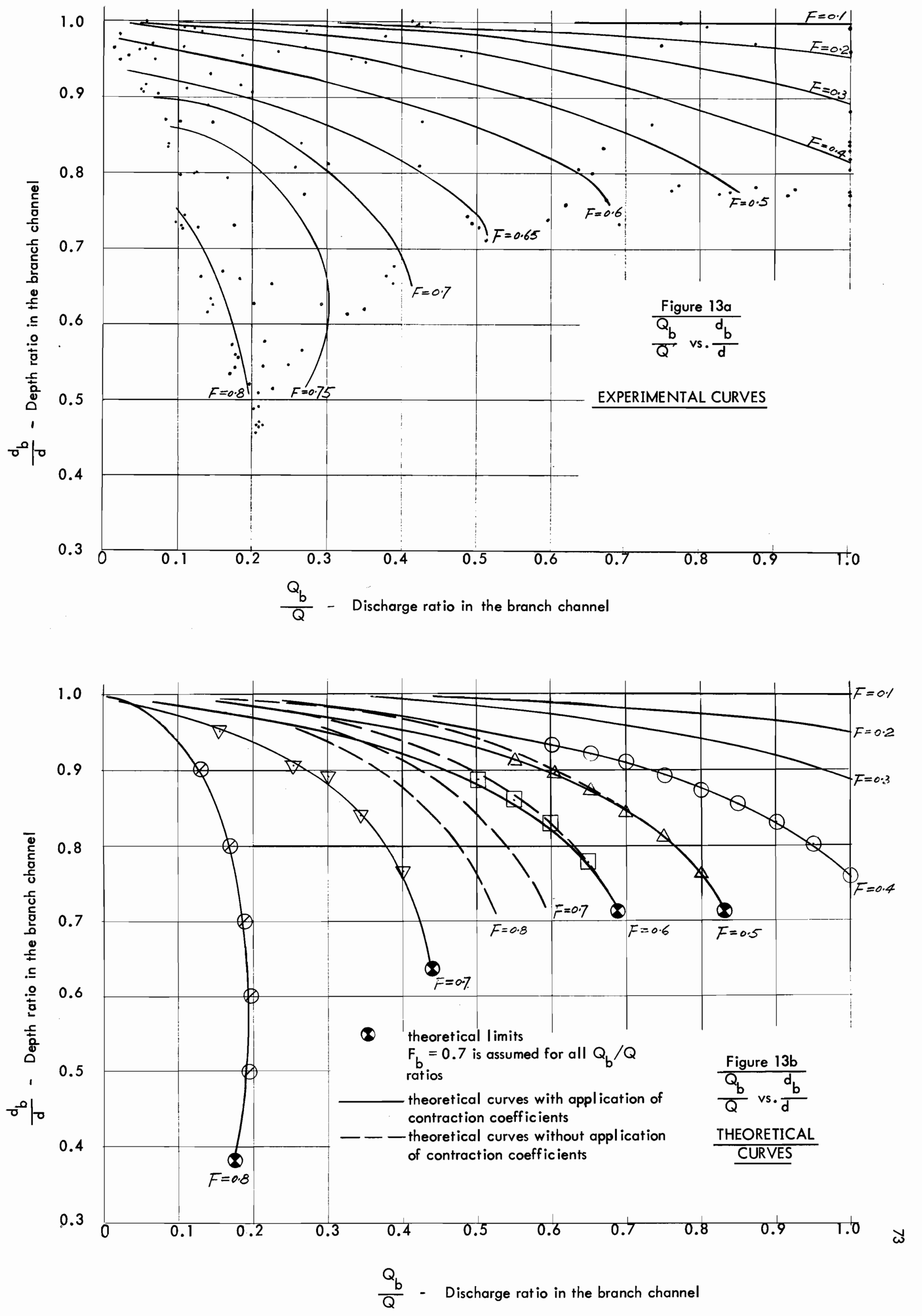
Figure 14

Flow in the branch channel - as influenced by the gate at the end of the main channel extension.

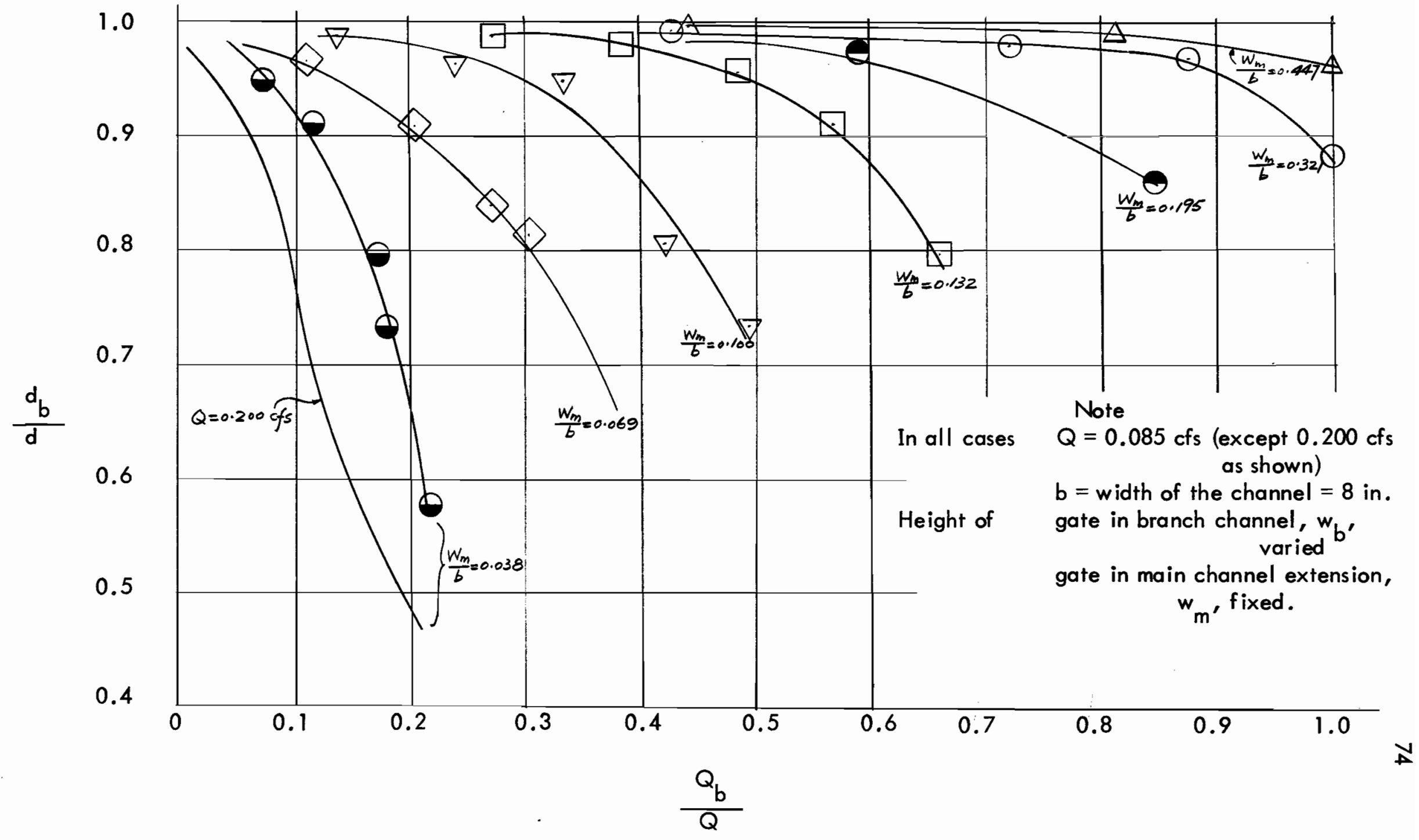




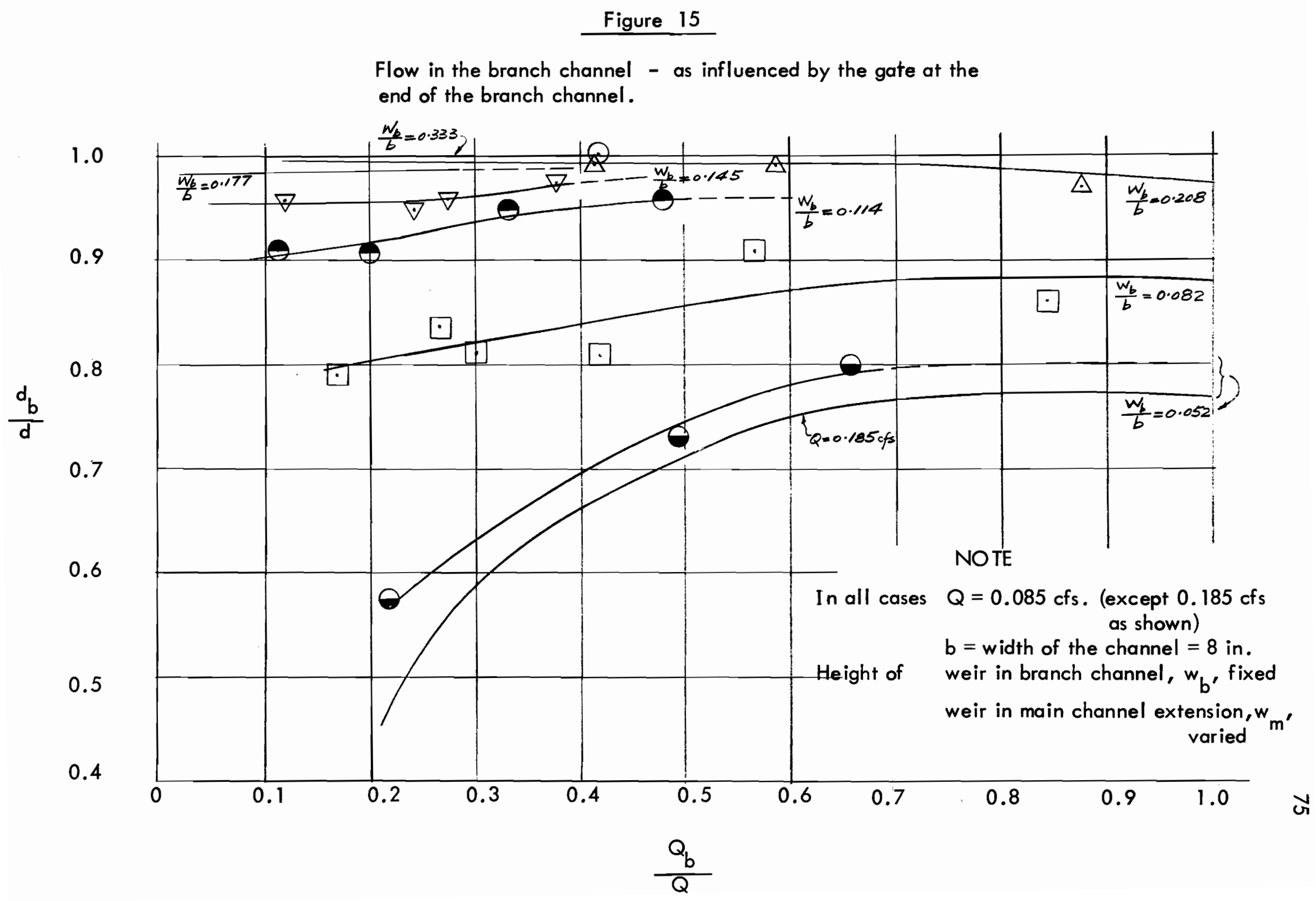




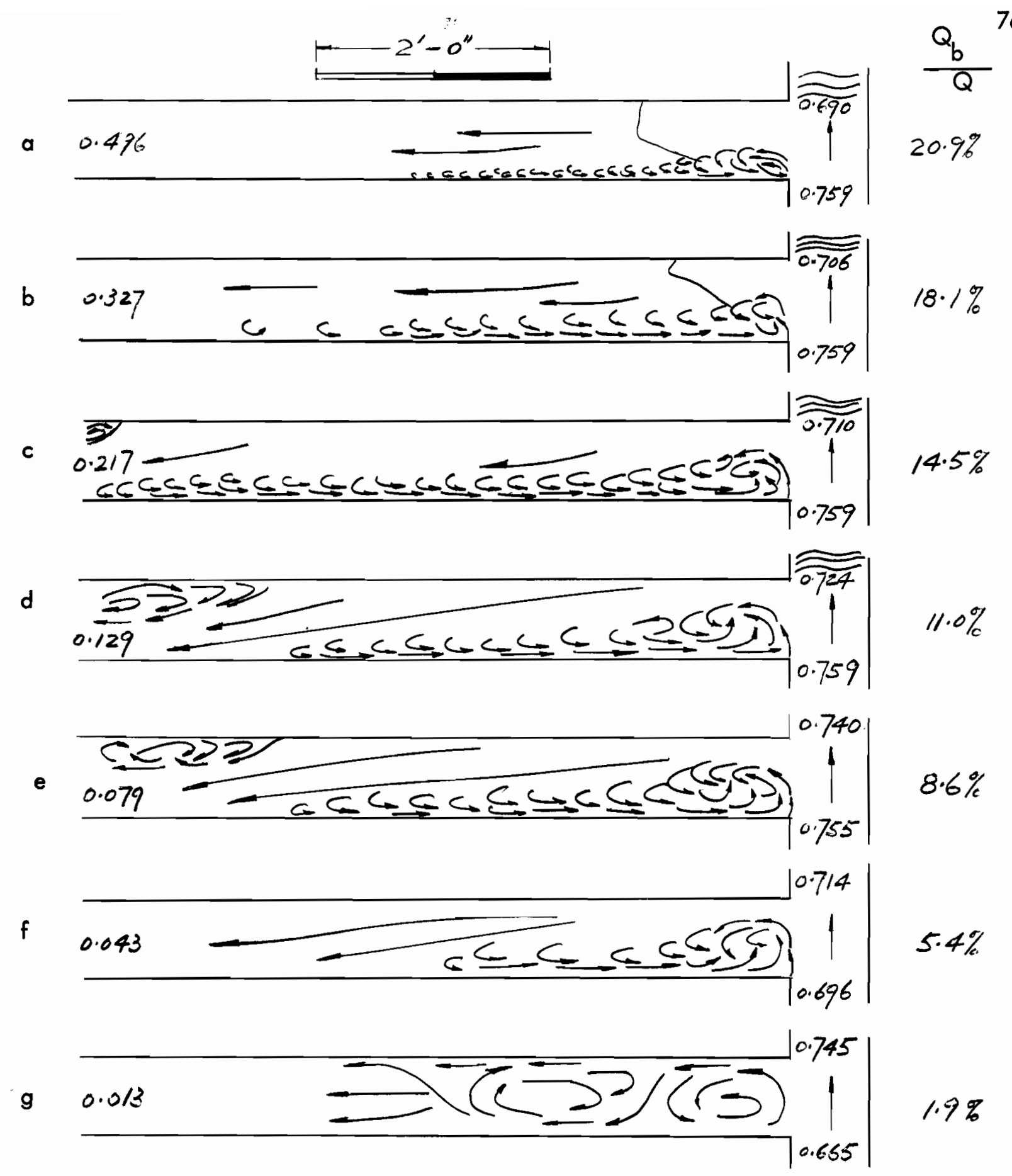

$\cong$ Hydraulic jumps in main channel extension

Figure 16

Development of recirculation regions in the branch channel, showing the observable flow directions. Numerical figures indicated in various sections of the channels are the respective Froude numbers. 


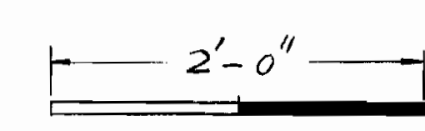

$\frac{Q_{b}}{Q}$

a

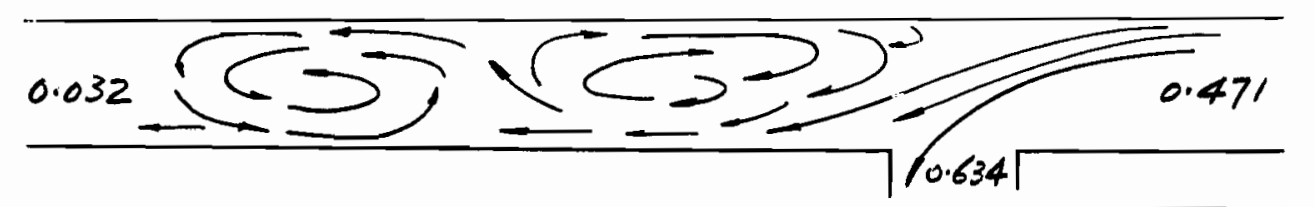

$92.6 \%$

b

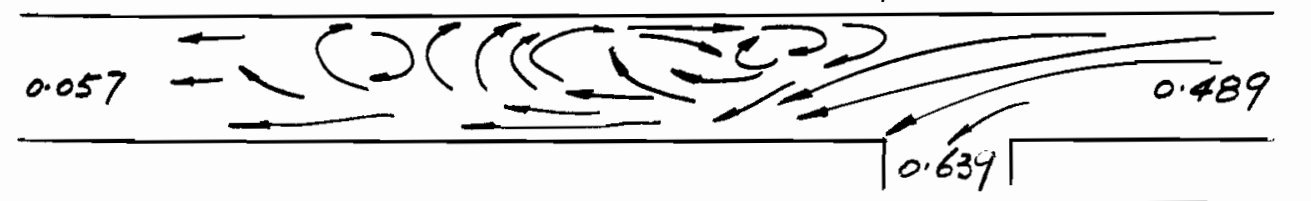

c

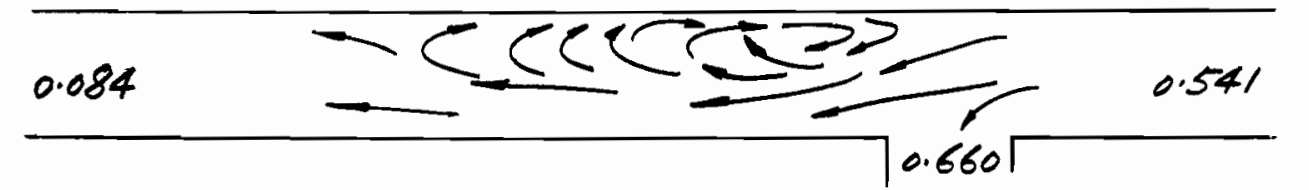

d

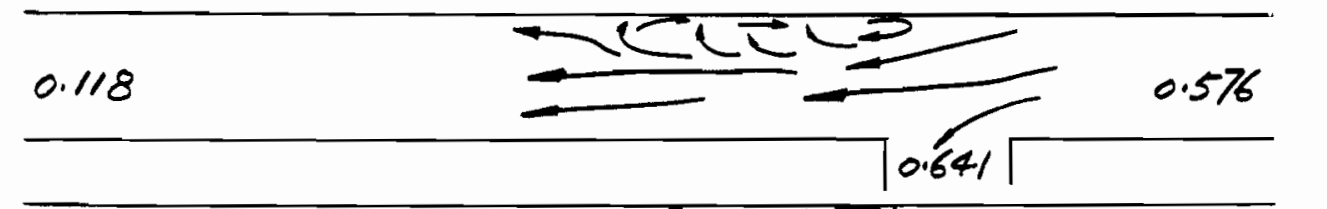

e

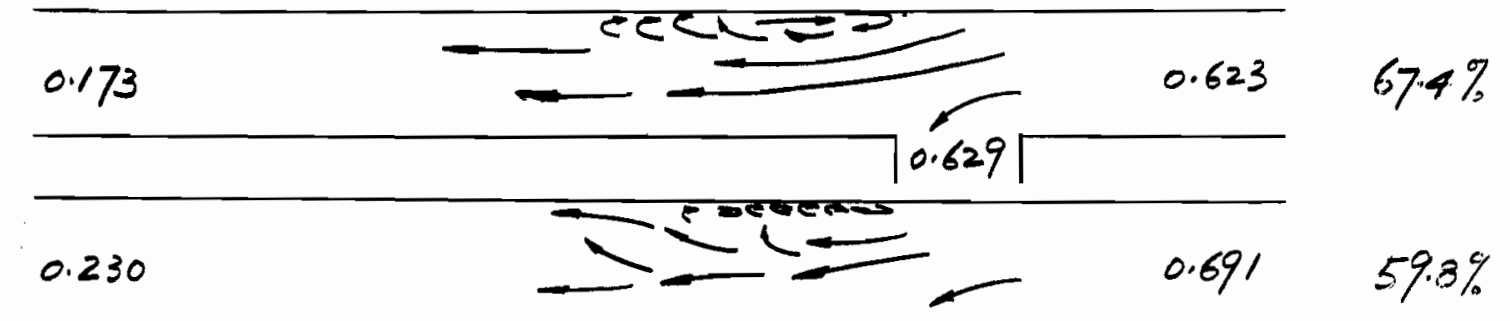

g

$\longrightarrow 0.631\rceil$

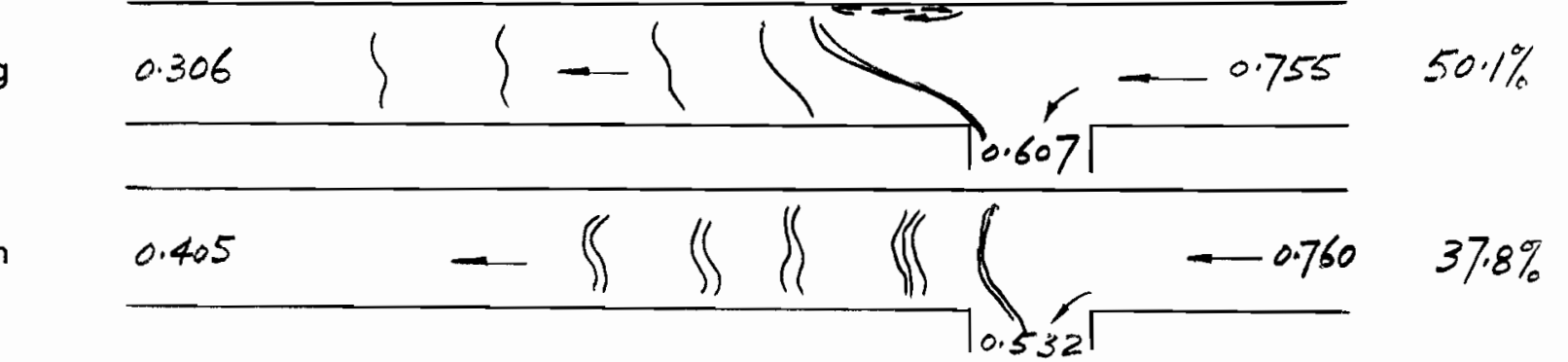
$\approx$ Hydraulic jumps in main channel extension.

Figure 17

Development of recirculation regions in the main channel extension, showing the observable flow directions. Numerical figures indicated in various sections of the channels are the respective Froude numbers. 


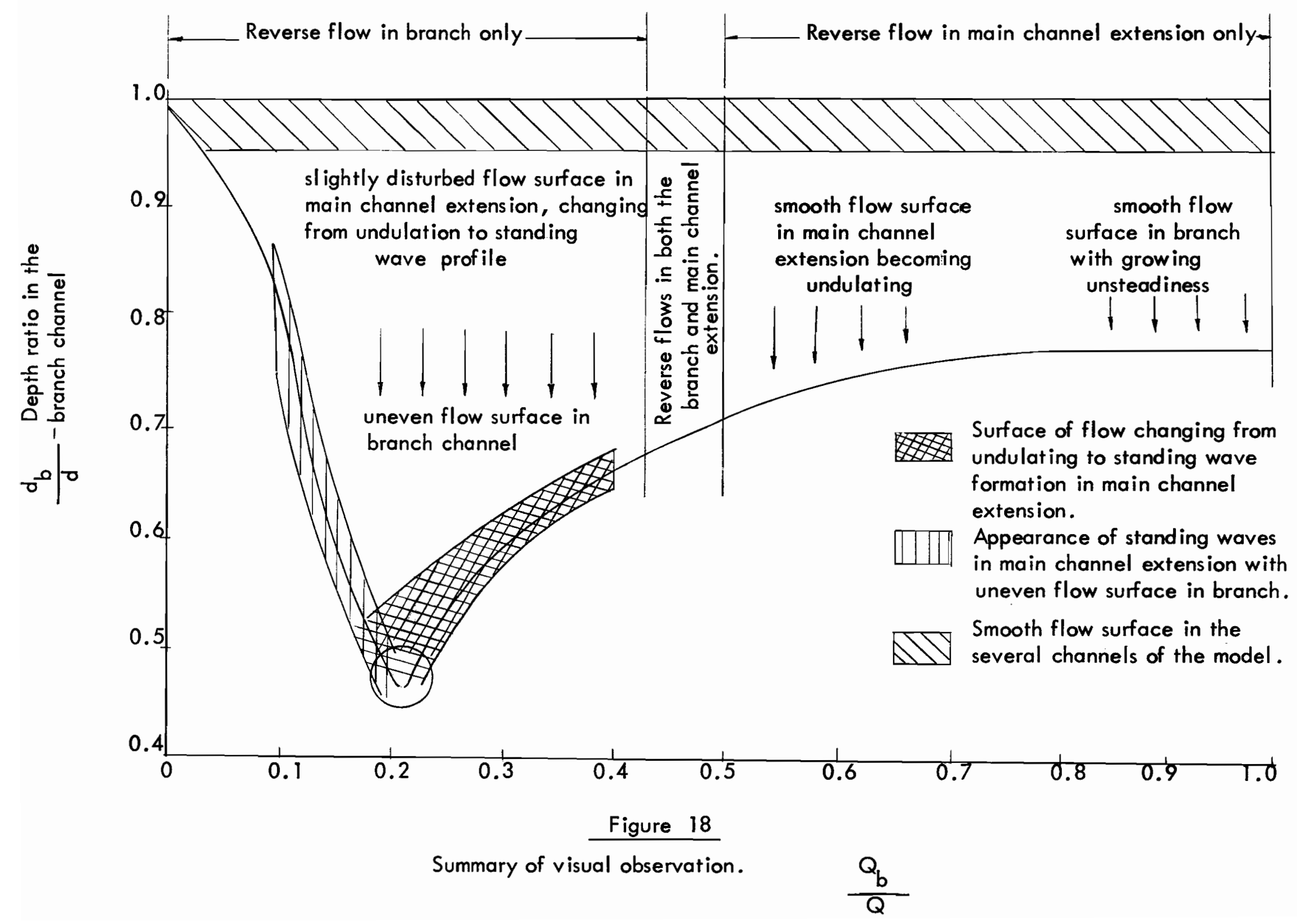




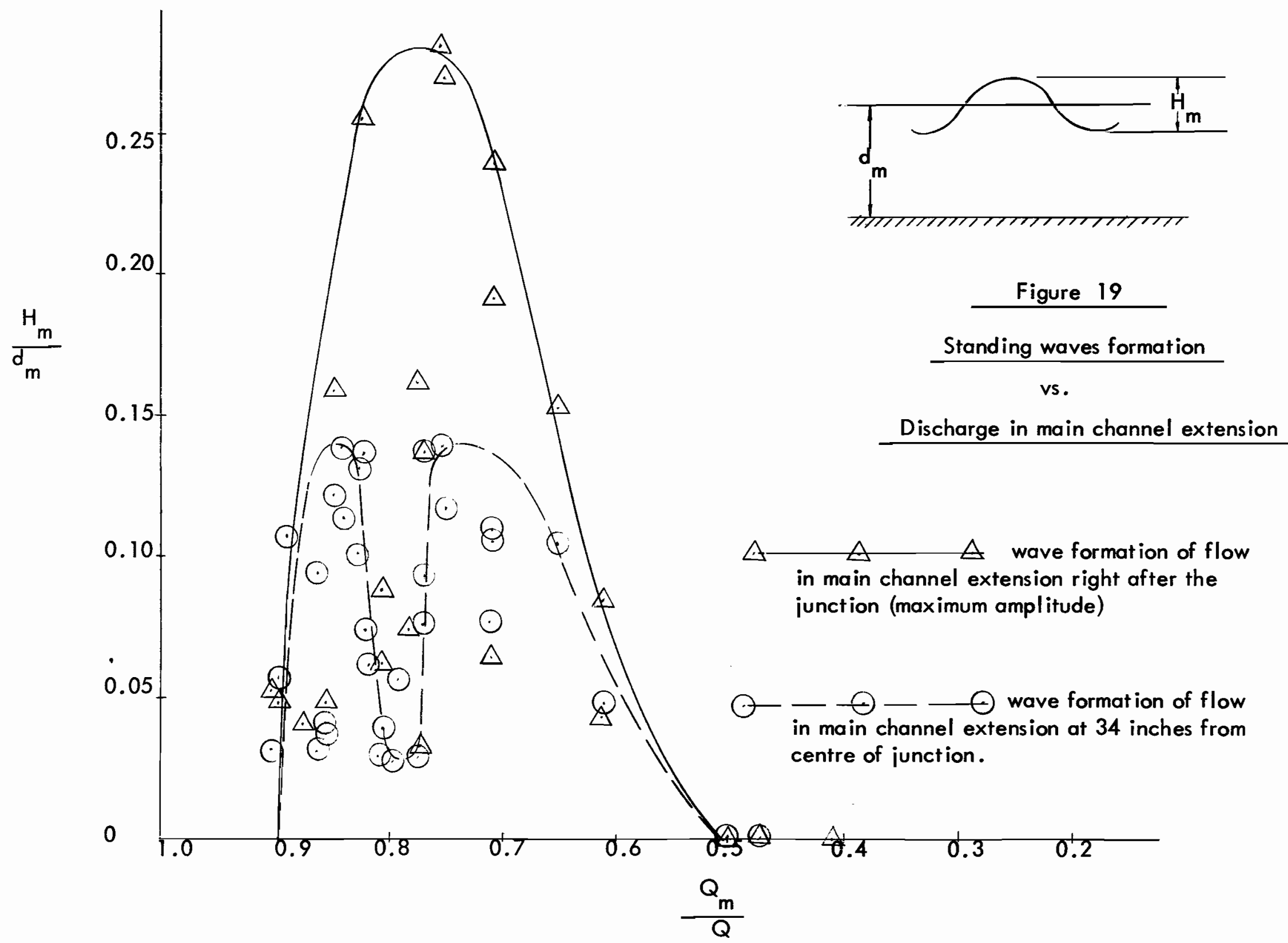




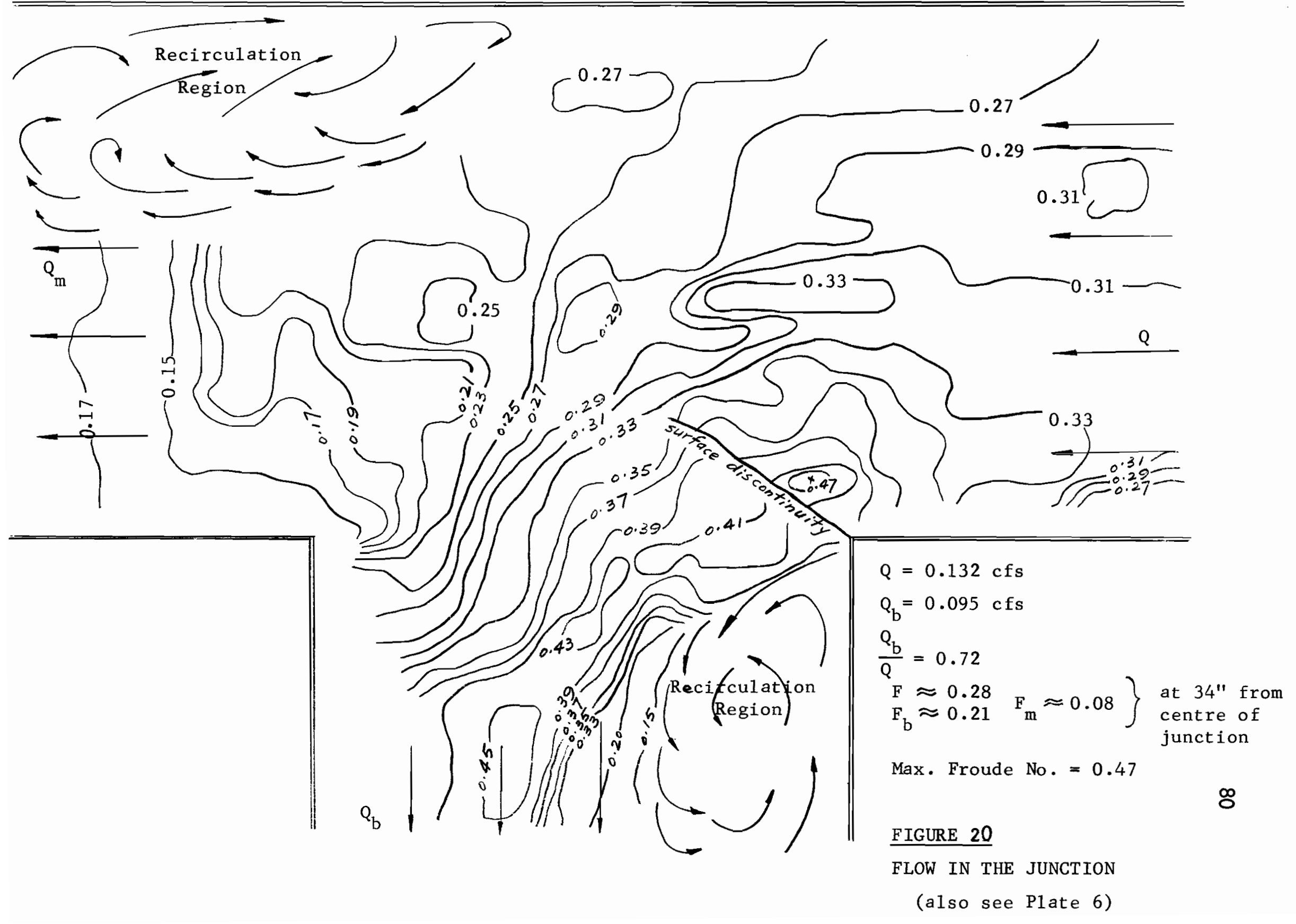




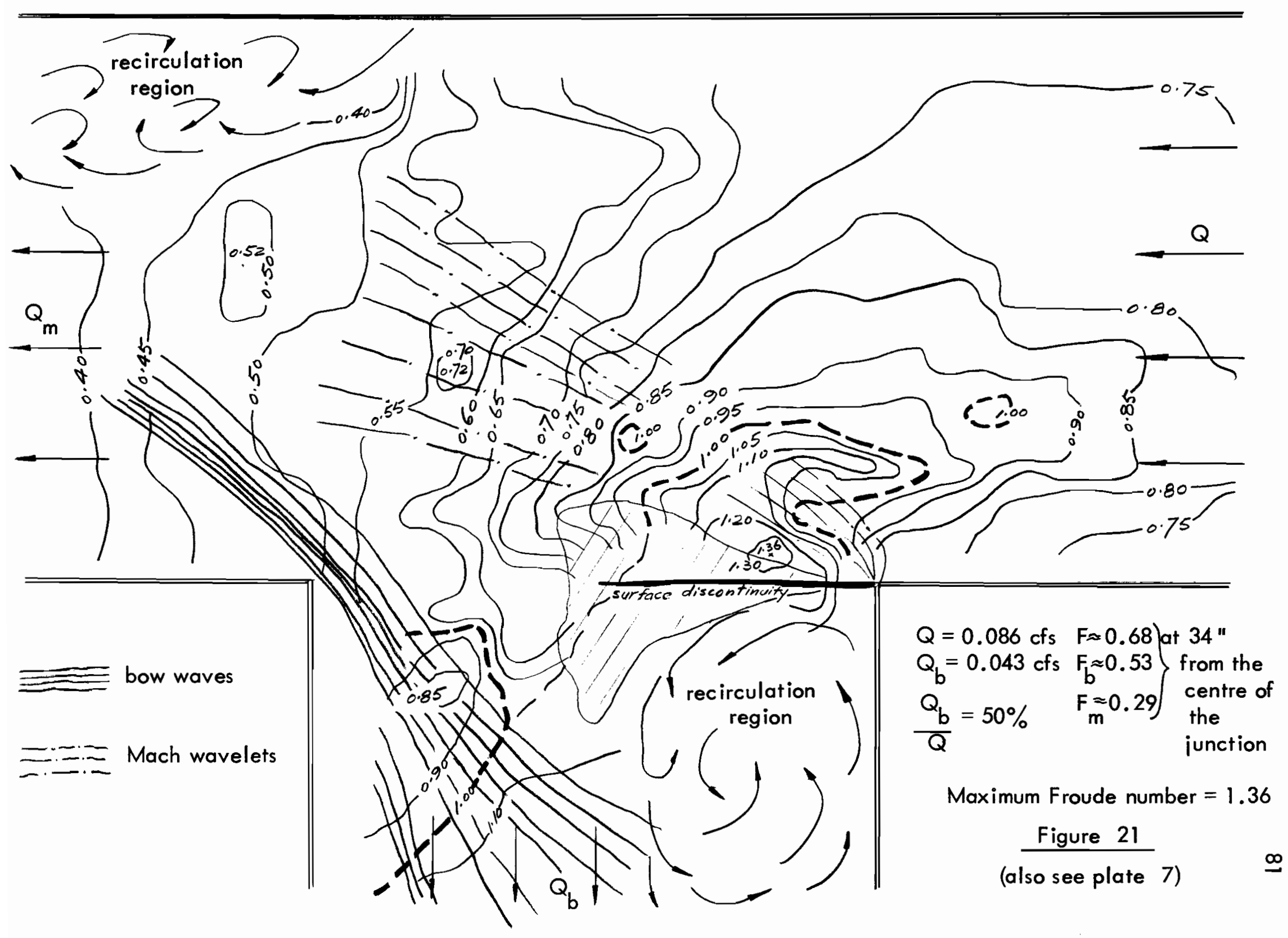




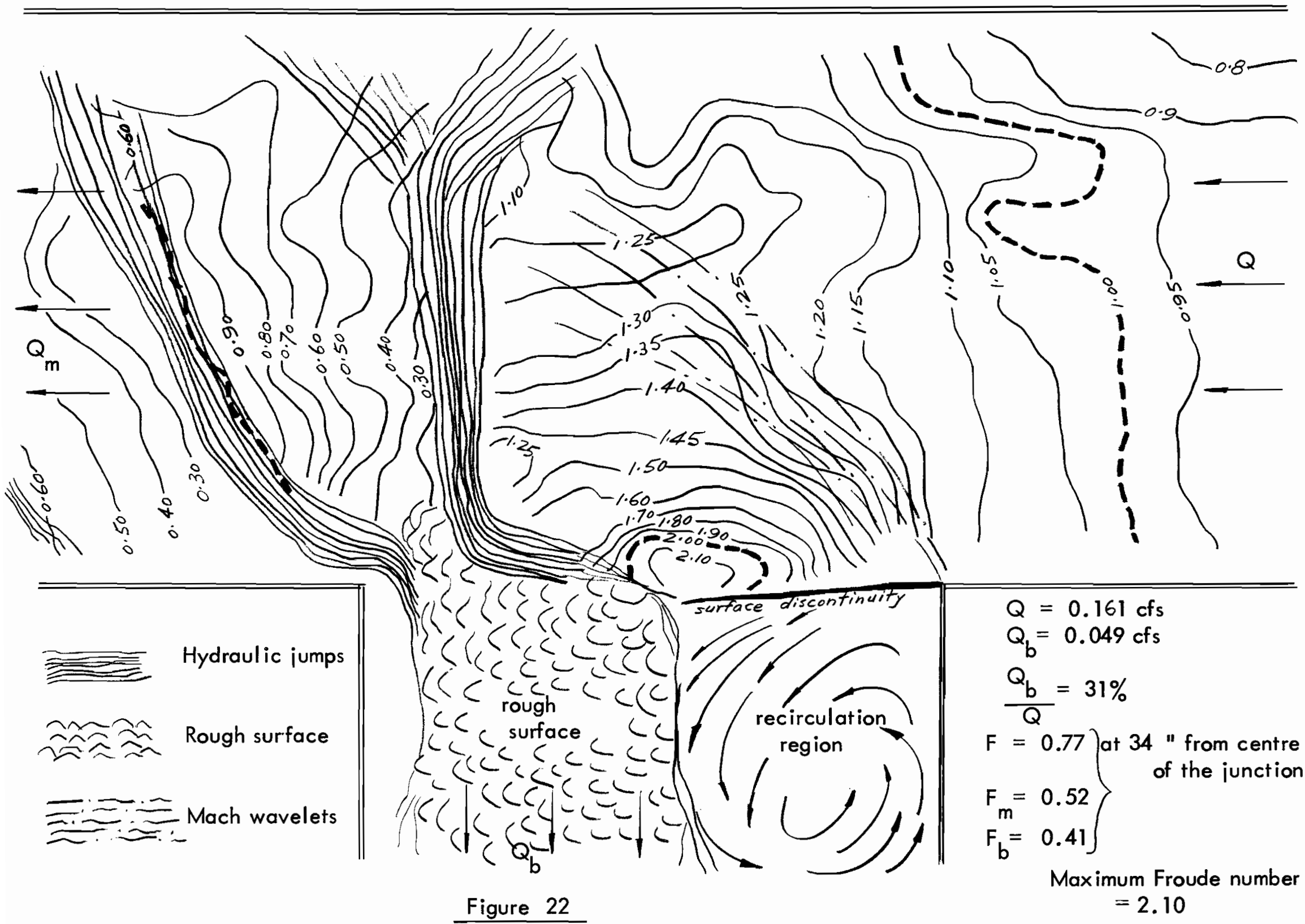

Flow in junction (also see plates 8 and 9) 
Figure 23

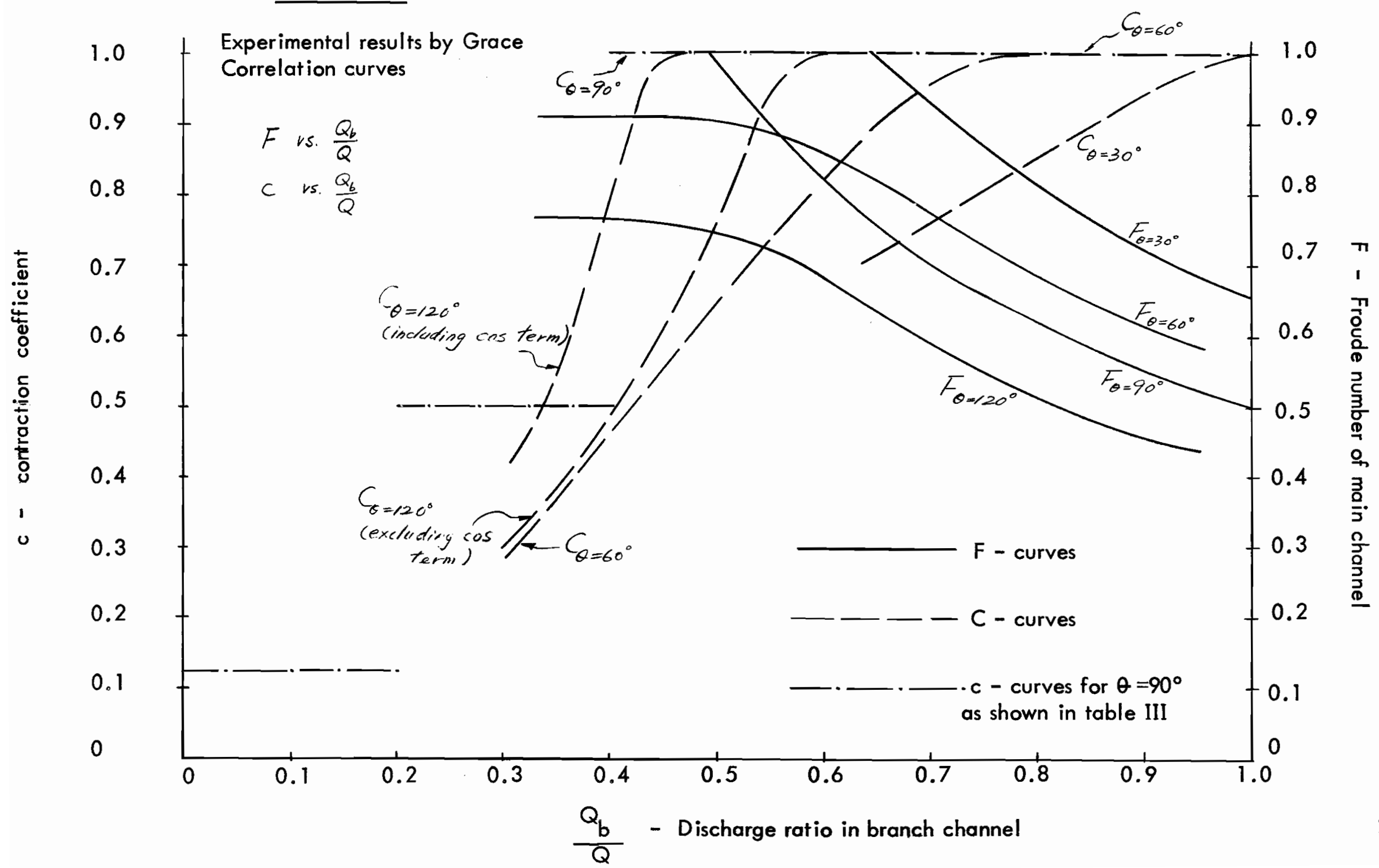


CAMERA POSITIONS

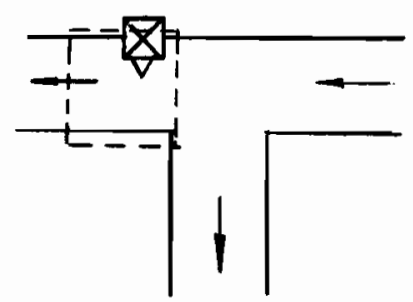

Plate 2

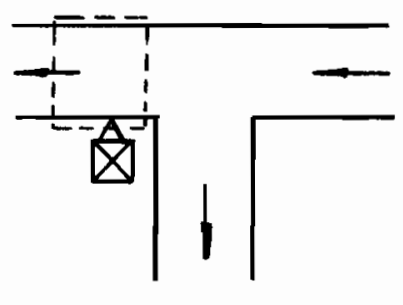

Plate 4

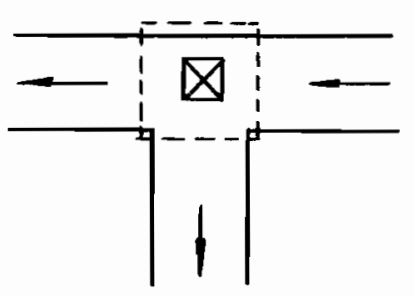

Plate 6

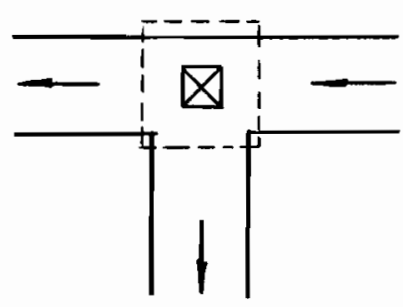

Plate 8

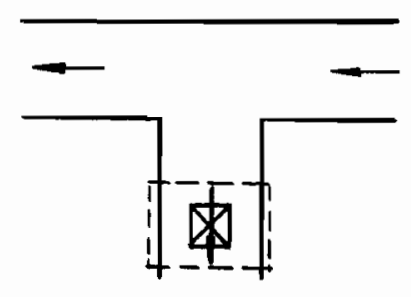

Plate 3

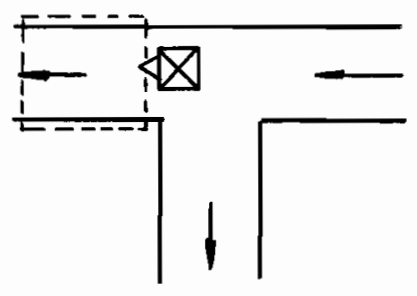

Plate 5

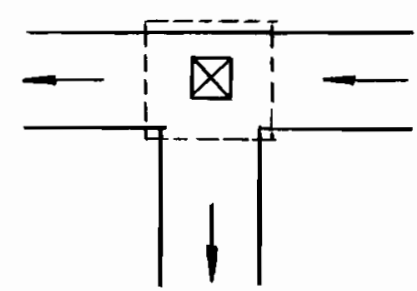

Plate 7

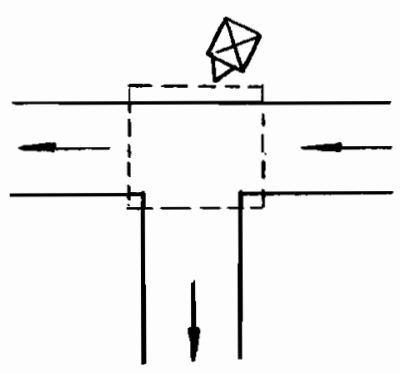

Plate 9 


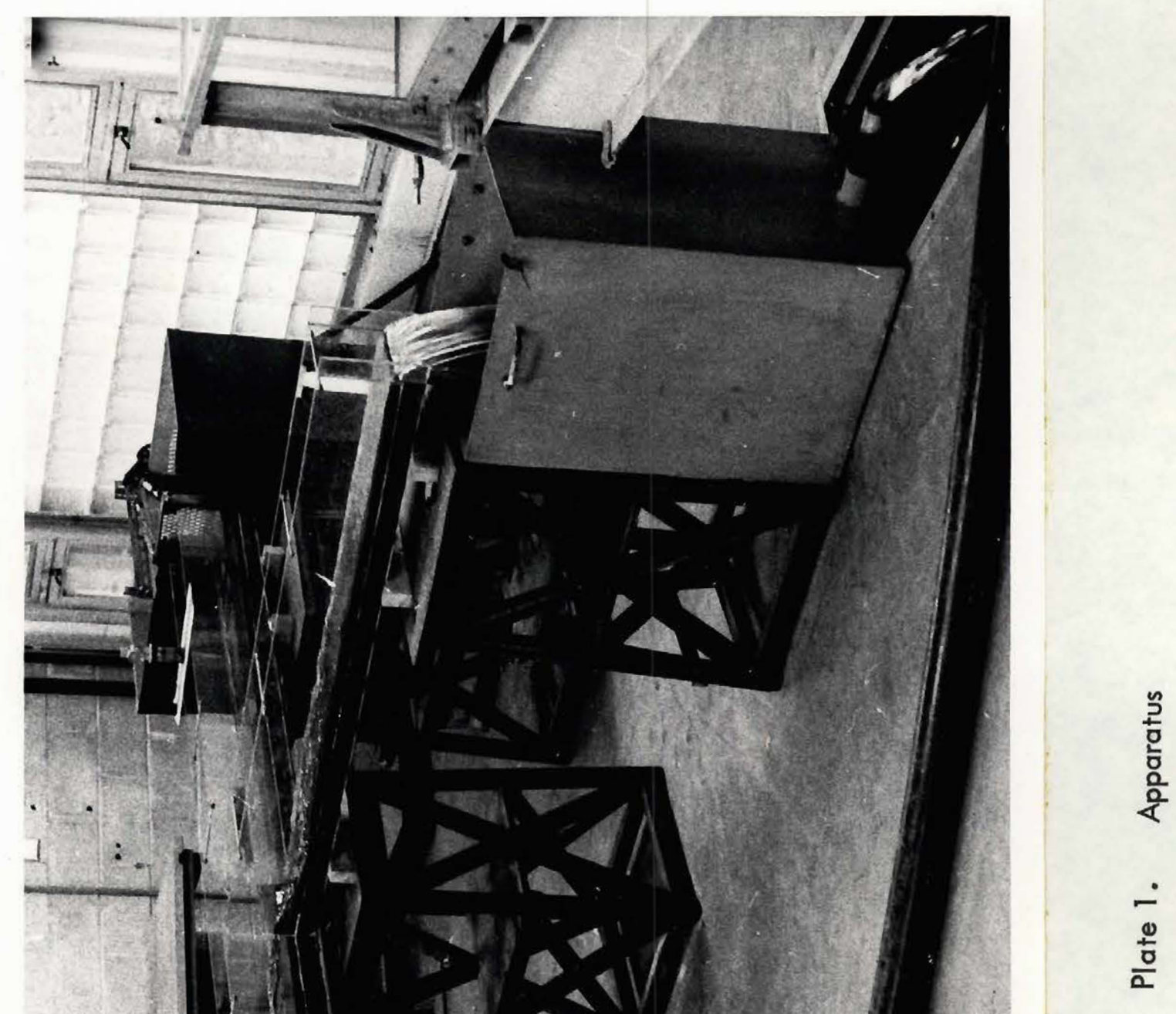




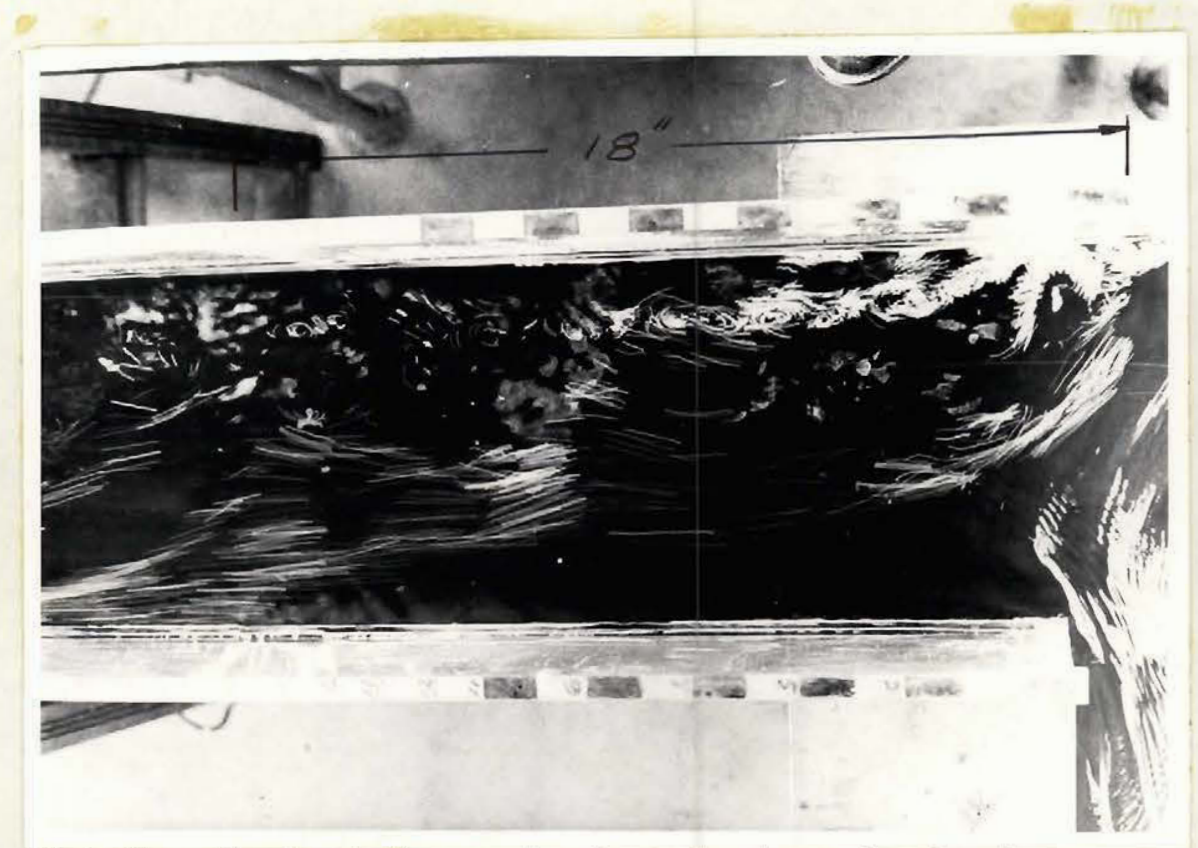

Plate 2. Recirculation region in main channel extension showing separation at junction and reattachment downstream. (Chalk powder showing surface streamlines on black channel bed.)

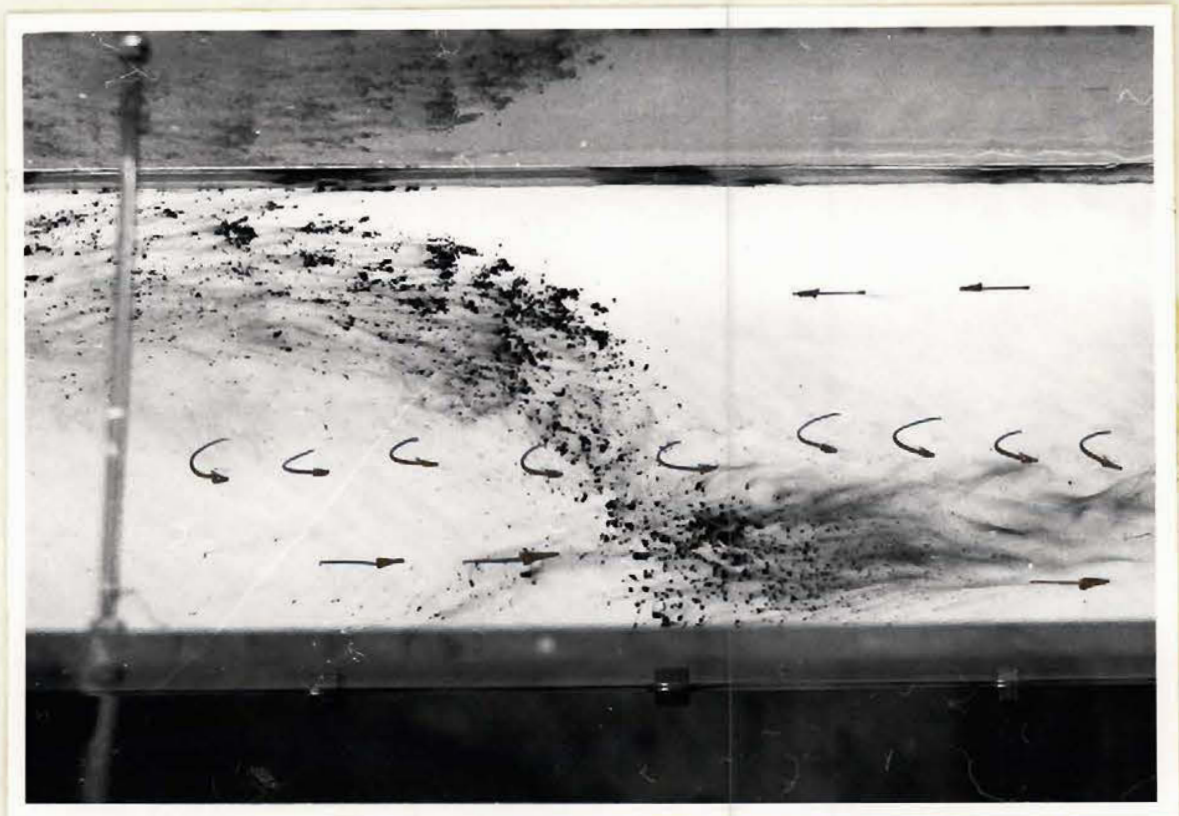

Plate 3. Reverse flow in branch channel. (Potassium permanganate powder showing bottom flow on white channel bed, seconds after being sprayed across the bottom of the channel.) 


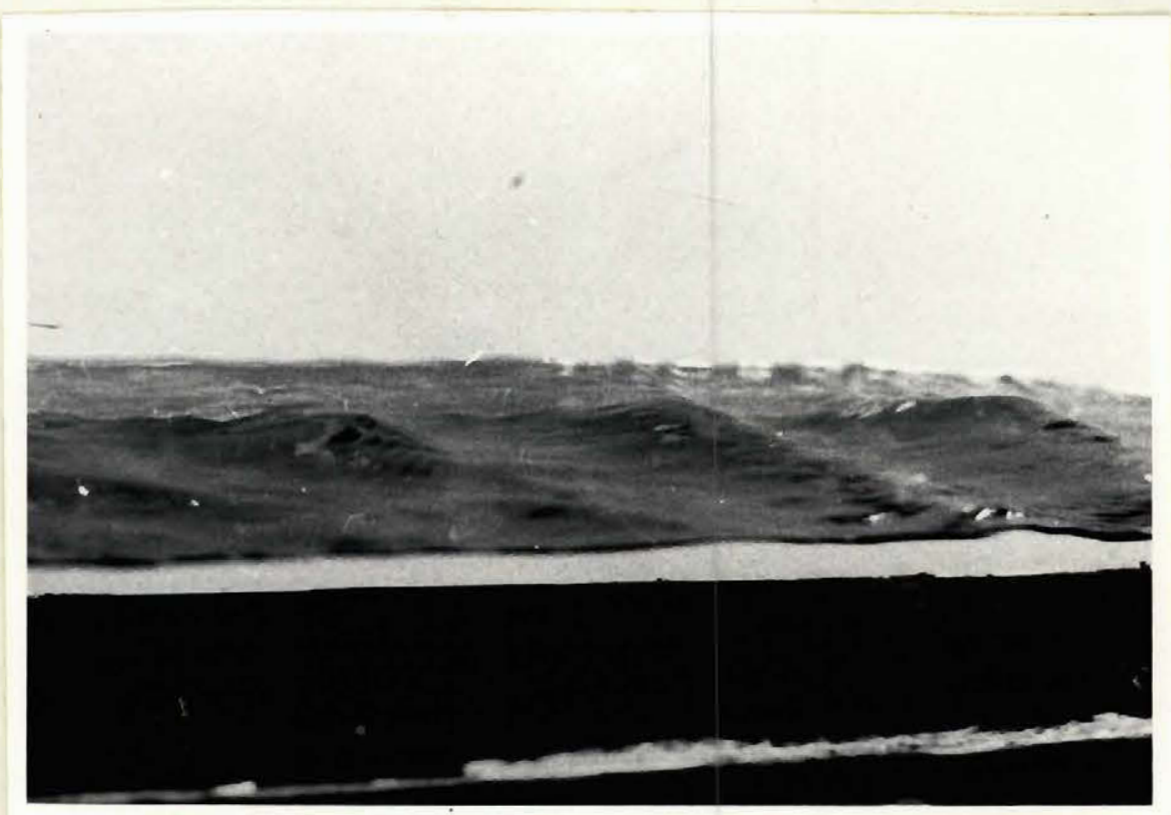

Plate 4. Standing waves in main channel extension (side view).

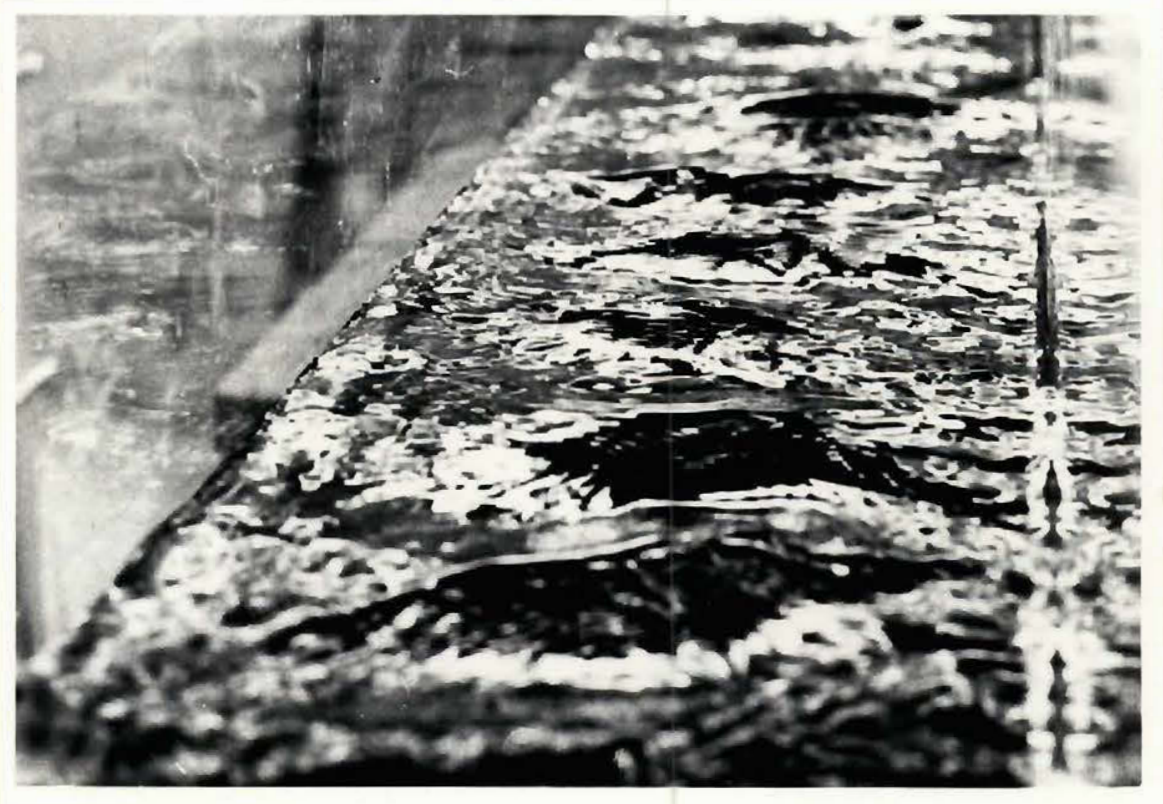

Plate 5. Standing waves in main channel extension (looking downstream). 


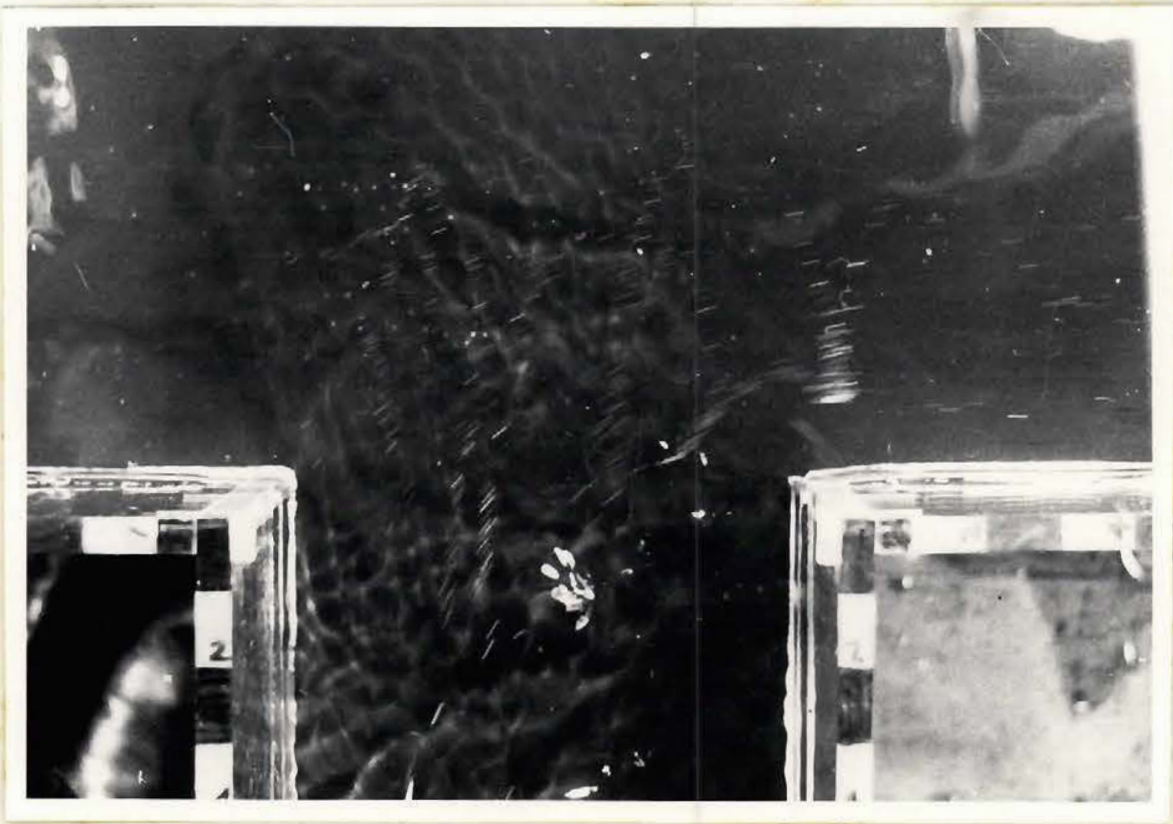

Plate 6. Flow in the junction (top view). $F=0.28, Q_{b} / Q=0.72$, (see also Fig. 20).

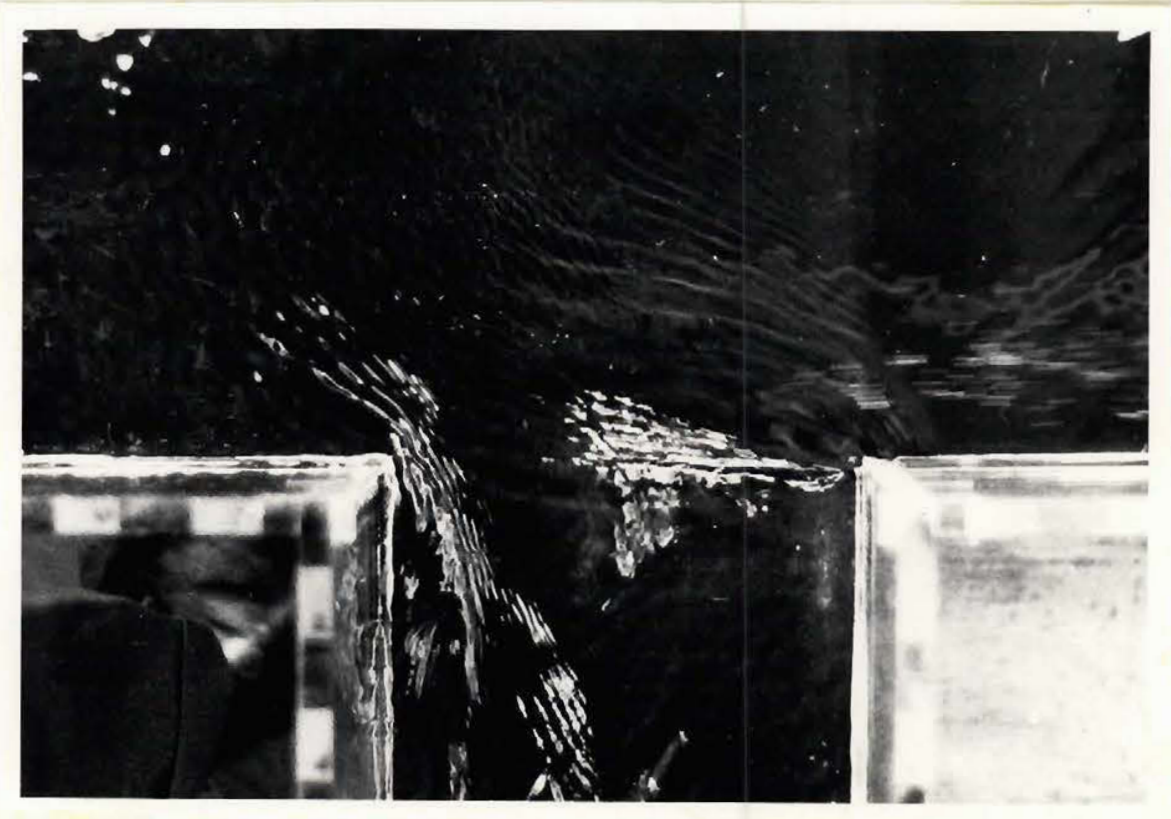

Plate 7. Flow in the junction showing Mach wavelets and bow waves in the upstream and downstream corners respectively (top view). $F=0.68, Q_{b} / Q=0.50$, (see also Fig. 21). 


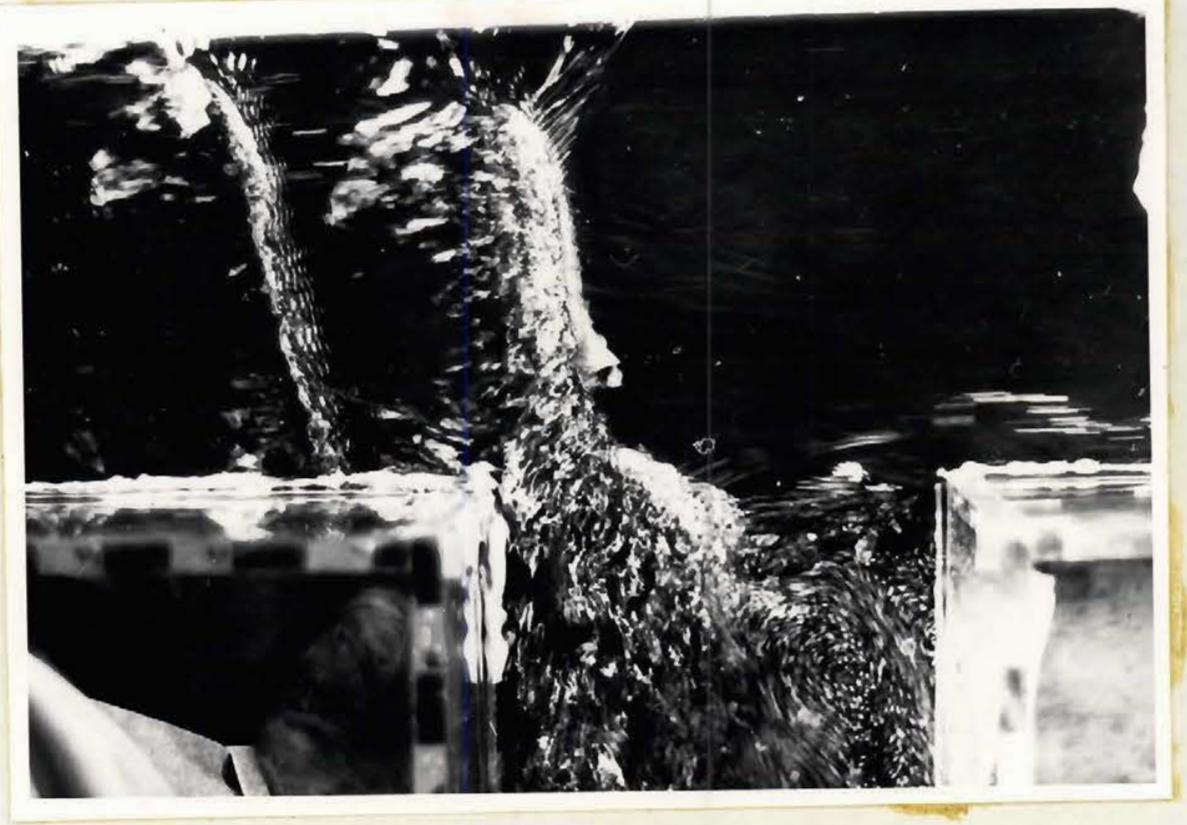

Plate 8. Flow in the junction showing hydraulic jumps and recirculation region with chalk powders (top view). $F=0.77, Q_{b} / Q=0.31$ (see also Fig. 22).

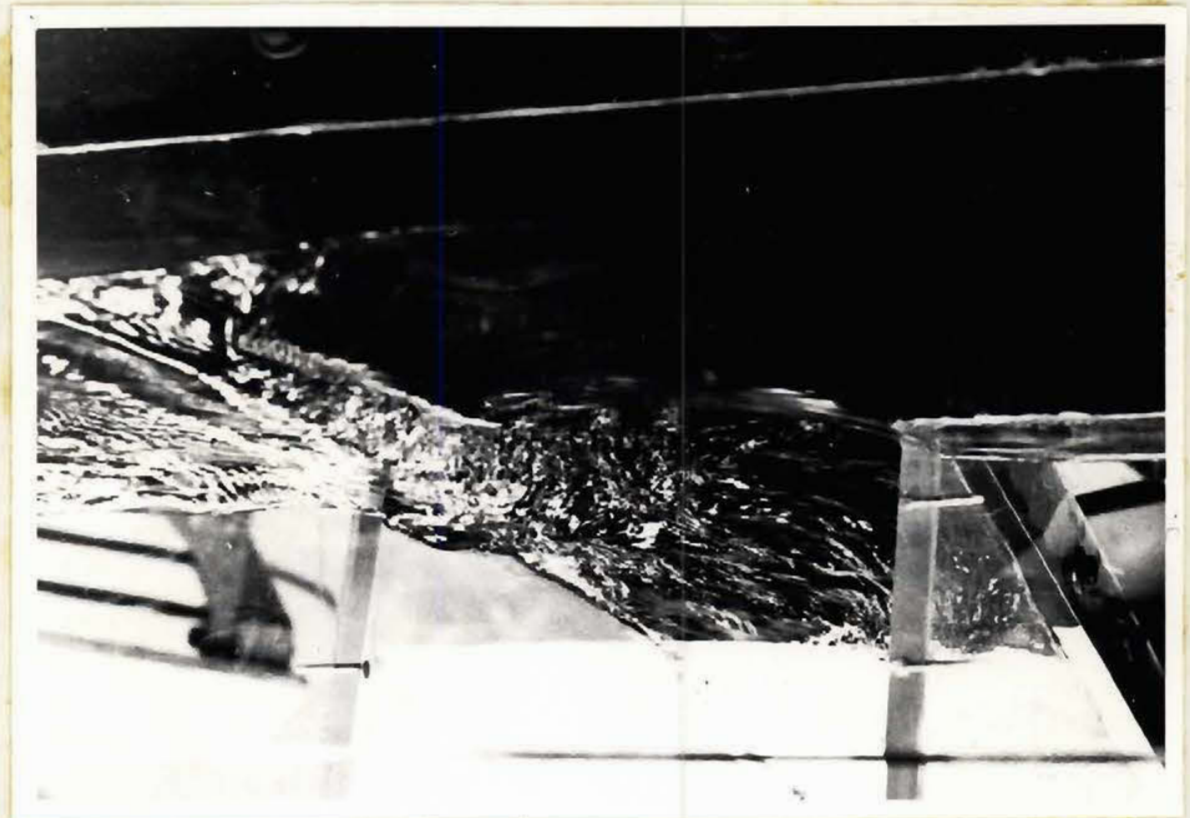

Plate 9. Flow in the junction (side view). $F=0.77, Q_{b} / Q=0.31$ (see also Fig. 22). 